\title{
EXISTENCE OF ANALYTIC SOLUTIONS FOR THE CLASSICAL STEFAN PROBLEM
}

\author{
JAN PRÜSS, JÜRGEN SAAL, AND GIERI SIMONETT
}

\begin{abstract}
We prove that under mild regularity assumptions on the initial data the two-phase classical Stefan problem admits a (unique) solution that is analytic in space and time.
\end{abstract}

\section{INTRODUCTION}

The Stefan problem is a model for phase transitions in liquid-solid systems and accounts for heat diffusion and exchange of latent heat in a homogeneous medium. The strong formulation of this model corresponds to a free boundary problem involving a parabolic diffusion equation for each phase and a transmission condition prescribed at the interface separating the phases.

In order to describe the physical situation in some more detail, we consider a domain $\Omega$ that is occupied by a liquid and a solid phase, say water and ice, that are separated by an interface $\Gamma$. Due to melting or freezing, the corresponding regions occupied by water and ice will change and, consequently, the interface $\Gamma$ will also change its position and shape. This leads to a free boundary problem.

The basic physical laws governing this process are conservation of mass and conservation of energy. The unknowns are the temperature $u^{+}$and $u^{-}$for the liquid and solid phase, respectively, and the position of the interface $\Gamma$ separating the two different phases. The conservation laws can then be expressed by a diffusion equation for $u^{+}$and $u^{-}$in the respective regions $\Omega^{+}$and $\Omega^{-}$occupied by the liquid and solid phase and by the so-called Stefan condition which accounts for the exchange of latent heat due to melting or solidifying. In the classical Stefan problem one assumes that

$$
u^{+}=u^{-}=0 \quad \text { on } \quad \Gamma
$$

where 0 is the melting temperature.

The Stefan problem has been studied in the mathematical literature for over a century, see [50, 45] and [56, pp. 117-120] for a historic account, and has attracted the attention of many mathematicians.

The Stefan problem is known to admit a unique global weak solution, provided the given data (that is, the initial temperature and the source terms) have the 'correct' signs; see for instance [29,31,37] and [41, pp. 496-503]. If the sign conditions are obstructed, then the Stefan problem becomes ill-posed [20].

In the one-dimensional case the Stefan problem has been extensively studied by many authors, among them J.R. Cannon, A. Friedmann, C.D. Hill, D.B. Kotlow,

Date: January 16, 2007. 
M. Primicerio, L.I Rubinstein, and D.G. Schaeffer, see $[28,30,50]$ for more information.

In the current paper we establish existence and regularity properties for solutions of the classical two-phase Stefan problem. The novelty is that we can prove that the free boundary $\Gamma$ and the temperature $u^{ \pm}$are analytic in space and time.

Results concerning the regularity of the free boundary for weak solutions of the multidimensional one-phase Stefan problem were established in [14, 15, 32, 38, 39], and continuity of the temperature was proved in [17]. The regularity results were derived by formulating the one-phase Stefan problem as a parabolic variational inequality $[22,32]$, for which the solution exists globally in time. In more detail, Friedman and Kinderlehrer [32] proved that under suitable (restrictive) assumptions on the initial data, the domain occupied by water remains star-shaped and the free boundary is Lipschitz-continuous for all times. Caffarelli [14, 15] showed that if for a fixed time $t_{0}$, the point $X_{0}$ is a point of density for the coincidence set, then the free boundary is a $C^{1}$-surface in a time-space neighborhood of $\left(t_{0}, X_{0}\right)$. Building on this result, Kinderlehrer and Nirenberg [38, 39] established that the free boundary is smooth, that is, analytic in the space variables and in the second Gevrey class for the time variable. In case the water region is star-shaped with respect to the unit sphere, the authors derived that the free boundary is jointly analytic in the space and time variables, provided the heat supplied is analytic and positive. Under a very weak assumption (on the heat source) Matano [43] proved that any weak solution (in the sense of [22]) eventually becomes smooth, and that $\Gamma(t)$ approaches the shape of a (growing) sphere. In order to prove that the solution is eventually classical, Matano shows that each point $X_{0}$ of the free boundary $\Gamma(t)$ has positive density with respect to the ice region if $t$ is sufficiently large. Such a property does not follow simply from local regularity analysis: a study of the geometric features of $\Gamma(t)$ in some global aspect is needed. The main tool involved is the plane-reflection method.

We note here that the formulation of the Stefan problem as a variational inequality does not seem to have a natural extension to the two-phase problem.

If the data are sufficiently smooth and satisfy high order (up to order 23) compatibility conditions, classical solutions on a small time interval were obtained by Hanzawa [34]. The approach relies on the Nash-Moser implicit function theorem, and leads to a loss of derivatives for the solution.

A unique local in time solution to the one-phase Stefan problem in Sobolev spaces of periodic functions was constructed by Frolova and Solonnikov in [52, 53]. Their result is based on the contraction mapping principle and on results for a linear model problem established in [33].

Existence and uniqueness for a one-phase Stefan-like problem was obtained in [23] by Escher.

Continuity of the temperature for weak solutions of the multidimensional two-phase Stefan problem was obtained in $[16,18,19,57]$, and continuity of the temperature distribution for an $m$-phase Stefan model with $m>2$ was studied in [21]. More recently, the regularity of the free boundary for weak (viscosity) solutions was studied in $[3,4,5,40]$. In more detail, Athanasopoulos, Caffarelli and Salsa $[3,4]$ consider viscosity solutions of parabolic two-phase transition problems whose boundary is 
locally given by a Lipschitz graph in space and time. It is shown in [3] that this assumption yields Lipschitz continuity for the temperature as well. If the problem satisfies a non-degeneracy condition, the authors show in [4] that the free boundary and the temperature are $C^{1}$. The non-degeneracy condition states, roughly speaking, that the heat fluxes are not vanishing simultaneously on the free boundary. The authors also show by a counter example that the free boundary may not regularize instantaneously in the absence of the non-degeneracy condition [4, section 10]. (They construct an example where a Lipschitz free boundary stays Lipschitz for some time, without regularizing.) In some cases, the validity of the non-degeneracy condition can be verified by global considerations, see Nochetto [46]. In [40], Koch shows that under the same non-degeneracy condition $C^{1}$-free boundaries are in fact $C^{\infty}$-smooth.

Local existence of classical solutions on a small time interval for the two-phase problem was first obtained by Meirmanov [44], provided that the initial data satisfy high order compatibility conditions. Classical solutions for the two-phase Stefan problem in Hölder spaces and weighted Hölder spaces (without loss of regularity) were obtained by Bazaliı̌, Bizhanova, Degtyarev, Solonnikov, and Radkevich, see $[6,7,8,9,10,11,12,13,49]$ for more information.

In this paper we prove under mild regularity assumptions on the initial data that (local in time) solutions to the two-phase Stefan problem are in fact analytic in space and time. In order to obtain our results we first establish $L_{p}$-maximal regularity results for an appropriate linear problem, that is, we establish the best possible estimates for the linearized problem. This, in turn, allows us to use the contraction principle to obtain a unique solution for the nonlinear problem. Moreover, our approach allows us to resort to the implicit function theorem to establish analyticity. In contrast to the results and techniques in $[4,5,40]$, which are conditionally dependent on the ability to verify that a given weak (viscosity) solution satisfies the 'non-degeneracy' condition at a given point $\left(t_{0}, X_{0}\right) \in \Gamma$, we formulate conditions on the initial data which allow us to simultaneously prove existence and regularity properties of solutions.

We should like to mention that the authors in $[52,53]$ obtain a solution for the one-phase problem with periodic conditions having the same maximal regularity properties as in the current paper. However, our approach and our techniques are different from those in [53], and our regularity results are completely new. It is clear that the one-phase problem is also covered by our approach.

After these general remarks we shall now introduce the precise mathematical model we are considering. We will, in fact, look at the special geometry where the free boundary is represented as the graph of a function.

Let us then consider a family $\Gamma=\{\Gamma(t): t \in(0, T)\}$ of hypersurfaces in $\mathbb{R}^{n+1}$, where each individual hypersurface is assumed to be a graph over $\mathbb{R}^{n}$, that is,

$$
\Gamma(t)=\operatorname{graph}(\rho(t))
$$

for some $\rho(t): \mathbb{R}^{n} \rightarrow \mathbb{R}$. Moreover, let $\Omega^{+}(t)$ and $\Omega^{-}(t)$ denote the domain above and below $\Gamma(t)$, respectively, that is,

$$
\Omega^{ \pm}(t):=\left\{(x, y) \in \mathbb{R}^{n} \times \mathbb{R}: \pm y> \pm \rho(t, x)\right\} .
$$


We set $\Omega(t):=\Omega^{+}(t) \cup \Omega^{-}(t)$ and consider the following problem: Given $\Gamma_{0}=$ $\operatorname{graph}\left(\rho_{0}\right)$ and $u_{0}: \Omega(0) \rightarrow \mathbb{R}$, determine a family $\Gamma=\{\Gamma(t): t \in(0, T)\}$ and a function

$$
u: \bigcup_{t \in(0, T)}(\{t\} \times \Omega(t)) \rightarrow \mathbb{R}
$$

such that

$$
\left\{\begin{aligned}
\left(\partial_{t}-c \Delta\right) u & =0 & & \text { in } \bigcup_{t \in(0, T)}(\{t\} \times \Omega(t)), \\
\gamma u & =0 & & \text { on } \bigcup_{t \in(0, T)}(\{t\} \times \Gamma(t)), \\
V & =-\left[c \partial_{\nu} u\right] & & \text { on } \bigcup_{t \in(0, T)}(\{t\} \times \Gamma(t)), \\
u(0) & =u_{0} & & \text { in } \Omega(0), \\
\Gamma(0) & =\Gamma_{0}, & &
\end{aligned}\right.
$$

where $\gamma$ stands for the trace operator, $V$ denotes the normal velocity of $\Gamma$, and $\nu$ is the unit normal vector, pointing into $\Omega^{+}(t)$. Given any function $v: \Omega(t) \rightarrow \mathbb{R}$, we write $v^{+}$and $v^{-}$for the restriction of $v$ to $\Omega^{+}(t)$ and $\Omega^{-}(t)$, respectively. Moreover, we admit the possibility of two different diffusion coefficients in $\Omega^{ \pm}(t)$, i.e. $c$ is given as

$$
c(t, x, y)= \begin{cases}c_{+}, & (x, y) \in \Omega^{+}(t) \\ c_{-}, & (x, y) \in \Omega^{-}(t)\end{cases}
$$

Using this notation, let $\left[c \partial_{\nu} u\right]$ denote the jump of the normal derivatives of $u$ across $\Gamma(t)$, that is,

$$
\left[c \partial_{\nu} u\right]:=c_{+} \gamma \partial_{\nu} u^{+}-c_{-} \gamma \partial_{\nu} u^{-} .
$$

Of course, $u_{0}$ is a given initial value for $u$ and $\Gamma_{0}$ describes the initial position of $\Gamma$.

To formulate our main result, let $W_{p}^{s}\left(\mathbb{R}^{n}\right), s \geq 0, p \in(1, \infty)$, denote the SobolevSlobodeckij spaces, cf. [55] (see also Section 3). Then we have

Theorem 1.1. Let $p>n+3$. Then there is a number $\eta_{0}>0$ such that the following holds: Given $\left(u_{0}, \rho_{0}\right) \in W_{p}^{2-2 / p}(\Omega(0)) \times W_{p}^{2-2 / p}\left(\mathbb{R}^{n}\right)$ with

$$
\gamma u_{0}^{ \pm}=0, \quad \pm u_{0}^{ \pm}>0 \quad \text { on } \quad \Omega^{ \pm}(0), \quad \alpha_{ \pm}:=\partial_{\nu} u_{0}^{ \pm}\left(0, \rho_{0}(0)\right)>0,
$$

and

$$
\left\|\rho_{0}\right\|_{\mathrm{BUC}^{1}\left(\mathbb{R}^{n}\right)} \leq \eta_{0}, \quad\left\|\partial_{\nu} u_{0}^{ \pm}-\alpha_{ \pm}\right\|_{\mathrm{BUC}\left(\Gamma_{0}\right)} \leq \eta_{0},
$$

there exists $T=T\left(u_{0}, \rho_{0}\right)$ and an analytic solution $(u, \Gamma)$, where $\Gamma(t)=\operatorname{graph}(\rho(t))$, for the Stefan problem (1.2). More precisely, we have that

$$
M=\bigcup_{t \in(0, T)}(\{t\} \times \Gamma(t)) \quad \text { is a real analytic manifold }
$$

and that $u^{ \pm} \in C^{\omega}\left(\bar{\Omega}_{T}^{ \pm}, \mathbb{R}\right)$, with $\bar{\Omega}_{T}^{ \pm}:=\left\{(t,(x, y)) \in(0, T) \times \mathbb{R}^{n+1}:(x, y) \in \bar{\Omega}^{ \pm}(t)\right\}$. The solution $\Theta^{*} u$ is unique in the class $\mathbb{E}_{T}$ specified in Section 4, where $\Theta$ is the transformation defined in Section 2.

Remark 1.2. For simplicity we formulated our results for the case that the free boundary is given as the graph of a function. We should emphasize that the situation of a general geometry can be reduced to the geometry of a graph by first fixing an appropriate reference manifold, and then using the method of localization. It can then be shown that the smallness condition (1.5) can always be satisfied by a judicious choice of a reference manifold close to $\Gamma_{0}$. By the parabolic maximum principle, the non-degeneracy condition (1.4) persists as long as the solution does not develop singularities. 
If the interface condition (1.1) is replaced by the Gibbs-Thomson correction

$$
u^{+}=u^{-}=\sigma \kappa \quad \text { on } \Gamma,
$$

where $\sigma$ is a positive constant, called the surface tension, and where $\kappa$ denotes the mean curvature of $\Gamma$, then the resulting problem is called the Stefan problem with Gibbs-Thomson correction or the Stefan problem with surface tension. We refer to [27] for more information and results.

Finally, we want to remark that this paper also serves as a preparation to a forthcoming paper of the authors on singular limits for the two-phase Stefan problem. More precisely, it will be proved that a strong solution of the Stefan problem with the Gibbs-Thomson correction (1.6) tends for $\sigma \rightarrow 0$ to the strong solution of the classical Stefan problem constructed in this paper.

\section{THE TRANSFORMED PROBLEM}

In what follows we write $\nabla_{x}$ for the gradient with respect to $x \in \mathbb{R}^{n}$, whereas $\nabla$ denotes the full gradient with respect to $(x, y) \in \mathbb{R}^{n} \times \mathbb{R}$. With the same meaning we use $\Delta_{x}$ and $\Delta$. Let $T>0$ and set $\dot{\mathbb{R}}:=\mathbb{R} \backslash\{0\}$ and $\dot{\mathbb{R}}^{n+1}:=\mathbb{R}^{n} \times \dot{\mathbb{R}}$. In analogy to the definition of $u^{ \pm}: \Omega(t)^{ \pm} \rightarrow \mathbb{R}$ for a function $u$ on $\Omega(t)$ we denote the restriction of a function $v: \dot{\mathbb{R}}^{n+1} \rightarrow \mathbb{R}$ to $\mathbb{R}_{+}^{n+1}$ and $\mathbb{R}_{-}^{n+1}$ by $v^{+}$and $v^{-}$, respectively, where $\mathbb{R}_{ \pm}^{n+1}:=\left\{x \in \mathbb{R}^{n+1}: \pm x_{n+1}>0\right\}$. We intent to transform the equations in $\Omega(t)$ into a problem in $\dot{\mathbb{R}}^{n+1}$. For this purpose we define

$$
\begin{aligned}
& \Theta:[0, T] \times \dot{\mathbb{R}}^{n+1} \rightarrow Q_{T}:=\bigcup_{t \in[0, T]}(\{t\} \times \Omega(t)), \\
& \Theta(t, x, y)=(t, \psi(t, x, y)):=\left(t, x, y+\rho_{E}(t, x, y)\right),
\end{aligned}
$$

where $\Gamma(t)=\operatorname{graph}(\rho(t))$ is defined in the last section and $\rho_{E}:[0, T] \times \dot{\mathbb{R}}^{n+1} \rightarrow \mathbb{R}$ is a suitable extension of $\rho:[0, T] \times \mathbb{R}^{n} \rightarrow \mathbb{R}$, to be defined later. A simple computation yields

$$
(\nabla \psi)^{-1}=\left(\begin{array}{cccc}
1 & \cdots & 0 & 0 \\
\vdots & \ddots & & \vdots \\
0 & & 1 & 0 \\
\frac{-\partial_{1} \rho_{E}}{1+\partial_{y} \rho_{E}} & \cdots & \frac{-\partial_{n} \rho_{E}}{1+\partial_{y} \rho_{E}} & \frac{1}{1+\partial_{y} \rho_{E}}
\end{array}\right) .
$$

This shows that suitable conditions on the function $\rho_{E}$ imply $\Theta$ to be a diffeomorphism. For instance, assuming $\rho_{E} \in C^{k}\left((0, T) \times \dot{\mathbb{R}}^{n+1}\right)$ and $\left\|\partial_{y} \rho_{E}\right\|_{\infty}<1$ results in

$$
\Theta \in \operatorname{Diff}^{k}\left((0, T) \times \dot{\mathbb{R}}^{n+1}, Q_{T}\right), \quad k \in \mathbb{N} \cup\{\infty, \omega\} .
$$

It is clear that at this point we do not know how regular $\rho$ and $\rho_{E}$ are. But in Sections 4 and 5 we will see that these functions are sufficiently regular to justify the transformation of (1.2) into a quasilinear system that we are going to present next. So for the remaining part of this section we just assume that $\rho$ and the extension $\rho_{E}$ are suitable functions, such that the following computations make sense.

We denote the push-forward and pull-back by

$$
u=\Theta_{*} v=v \circ \Theta^{-1} \text { and } \quad v=\Theta^{*} u=u \circ \Theta,
$$


respectively. Note that

$$
\begin{aligned}
\Theta^{*} \partial_{t} u & =\partial_{t} v-\frac{\partial_{t} \rho_{E}}{1+\partial_{y} \rho_{E}} \partial_{y} v \\
\Theta^{*} \nabla u & =\Theta^{*} \nabla \Theta_{*} v=(\nabla \psi)^{-T} \nabla v \\
\Theta^{*} \Delta u & =\Theta^{*} \nabla^{T} \nabla u=\left\{(\nabla \psi)^{-T} \nabla\right\}^{T}(\nabla \psi)^{-T} \nabla v=: \Delta_{\Theta} v,
\end{aligned}
$$

where we mean $M^{-T}=\left(M^{-1}\right)^{T}$ for a matrix $M$. In slight abuse of notation we will also denote the pull-back $\Theta^{*} c$ of 'the diffusion coefficient $c$ ' introduced in (1.3) by $c$, that is, we set

$$
c(x, y)= \begin{cases}c_{+}, & y>0, \\ c_{-}, & y<0 .\end{cases}
$$

Explicitely, the tranformed Laplacian is therefore given by

$$
\begin{aligned}
& \Delta_{\Theta} v=\Delta_{x} v+\frac{1+\left|\nabla_{x} \rho_{E}\right|^{2}}{\left(1+\partial_{y} \rho_{E}\right)^{2}} \partial_{y}^{2} v-\frac{2\left\langle\nabla_{x} \rho_{E} \mid \nabla_{x} \partial_{y} v\right\rangle}{1+\partial_{y} \rho_{E}} \\
& -\frac{1}{1+\partial_{y} \rho_{E}}\left\{\Delta_{x} \rho_{E}+\frac{1+\left|\nabla_{x} \rho_{E}\right|^{2}}{\left(1+\partial_{y} \rho_{E}\right)^{2}} \partial_{y}^{2} \rho_{E}-\frac{2\left\langle\nabla_{x} \rho_{E} \mid \nabla_{x} \partial_{y} \rho_{E}\right\rangle}{1+\partial_{y} \rho_{E}}\right\} \partial_{y} v,
\end{aligned}
$$

where $\langle\cdot \mid \cdot\rangle$ denotes the standard scalar product in $\mathbb{R}^{n+1}$. Next let us transform the Stefan condition. Clearly, the function

$$
X(t, x):=(x, \rho(t, x)), \quad(t, x) \in[0, T] \times \mathbb{R}^{n},
$$

is a parametrization of $\Gamma(t)$ and

$$
\nu(t, X(t, x))=\frac{\left(-\nabla_{x} \rho(t, x), 1\right)}{\sqrt{1+\left|\nabla_{x} \rho(t, x)\right|^{2}}}
$$

is the outer unit normal at $\Gamma(t)$ pointing into $\Omega_{+}$. Thus, the Stefan condition

$$
V=\left\langle\partial_{t} X \mid \nu\right\rangle=-\left\langle c_{+} \gamma \nabla u^{+} \circ X-c_{-} \gamma \nabla u^{-} \circ X \mid \nu\right\rangle
$$

becomes

$$
\begin{aligned}
\partial_{t} \rho & =\left\langle\partial_{t} X \mid\left(-\nabla_{x} \rho, 1\right)\right\rangle=-\left\langle c_{+} \gamma \nabla u^{+} \circ X-c_{-} \gamma \nabla u^{-} \circ X \mid\left(-\nabla_{x} \rho, 1\right)\right\rangle \\
& =-\left\langle c_{+} \gamma \Theta^{*} \nabla u^{+}-c_{-} \gamma \Theta^{*} \nabla u^{-} \mid\left(-\nabla_{x} \rho, 1\right)\right\rangle \\
& =-\left\langle c_{+} \gamma \nabla v^{+}-c_{-} \gamma \nabla v^{-} \mid(\gamma \nabla \psi)^{-1}\left(-\nabla_{x} \rho, 1\right)\right\rangle .
\end{aligned}
$$

Here $\gamma$ is the restriction (trace) operator of $\mathbb{R}_{ \pm}^{n+1}$ onto $\mathbb{R}^{n} \equiv \mathbb{R}^{n} \times\{0\}$. Then equations (1.2) are formally equivalent to the system

$$
\left\{\begin{aligned}
\partial_{t} v-\frac{\partial_{t} \rho_{E}}{1+\partial_{y} \rho_{E}} \partial_{y} v-c \Delta_{\Theta} v & =0 & & \text { in } J \times \dot{\mathbb{R}}^{n+1}, \\
\gamma v^{ \pm} & =0 & & \text { on } J \times \mathbb{R}^{n}, \\
\partial_{t} \rho+\left\langle c_{+} \gamma \nabla v^{+}-c_{-} \gamma \nabla v^{-} \mid(\gamma \nabla \psi)^{-1}\left(-\nabla_{x} \rho, 1\right)\right\rangle & = & & \text { on } J \times \mathbb{R}^{n}, \\
v(0)=\left.\Theta^{*}\right|_{t=0} u(0) & = & v_{0} & \text { in } \mathbb{R}^{n+1}, \\
\rho(0) & = & \rho_{0} & \text { in } \mathbb{R}^{n},
\end{aligned}\right.
$$

where $J=[0, T]$. Finally, we rephrase this system as

$$
\left\{\begin{aligned}
\left(\partial_{t}-c \Delta\right) v & =F\left(v, \rho_{E}\right) & & \text { in } J \times \dot{\mathbb{R}}^{n+1} \\
\gamma v^{ \pm} & =0 & & \text { on } J \times \mathbb{R}^{n} \\
\partial_{t} \rho+\left[c \gamma \partial_{y}\left(v-a \rho_{E}\right)\right] & =H\left(v, \rho_{E}\right) & & \text { on } J \times \mathbb{R}^{n} \\
v(0) & =v_{0} & & \text { in } \mathbb{R}^{n+1} \\
\rho(0) & =\rho_{0} & & \text { in } \mathbb{R}^{n}
\end{aligned}\right.
$$


and we will require that the function $\rho_{E}$ satisfies the equation

$$
\left\{\begin{aligned}
\left(\partial_{t}-c \Delta\right) \rho_{E} & =0 & & \text { in } J \times \dot{\mathbb{R}}^{n+1}, \\
\gamma \rho_{E}^{ \pm} & =\rho & & \text { on } J \times \mathbb{R}^{n}, \\
\rho_{E}(0) & =e^{-|y|\left(1-\Delta_{x}\right)^{\frac{1}{2}}} \rho_{0} & & \text { in } \dot{\mathbb{R}}^{n+1} .
\end{aligned}\right.
$$

The coefficient $a$ in equation (2.5) is, by definition, given by

$$
a(x, y)=\left\{\begin{array}{ll}
a_{+}, & y>0, \\
a_{-}, & y<0,
\end{array} \quad \text { where } \quad a_{ \pm}:=\frac{\gamma \partial_{y} v_{0}^{ \pm}(0)}{1+\gamma \partial_{y} \rho_{E}^{ \pm}(0,0)} .\right.
$$

Observe that $\alpha_{ \pm}>0$, given in (1.4), implies that also

$$
a_{ \pm}=\frac{\gamma \partial_{y} v_{0}^{ \pm}(0)}{1+\gamma \partial_{y} \rho_{E}^{ \pm}(0,0)}=\left(1+\left|\nabla_{x} \rho_{0}(0)\right|^{2}\right)^{-1 / 2} \partial_{\nu} u_{0}^{ \pm}\left(0, \rho_{0}(0)\right)>0 .
$$

Furthermore, we have

$$
\begin{aligned}
{\left[c \gamma \partial_{y}\left(v-a \rho_{E}\right)\right] } & =c_{+} \gamma \partial_{y}\left(v-a \rho_{E}\right)^{+}-c_{-} \gamma \partial_{y}\left(v-a \rho_{E}\right)^{-} \\
& =c_{+} \gamma \partial_{y}\left(v^{+}-a_{+} \rho_{E}^{+}\right)-c_{-} \gamma \partial_{y}\left(v^{-}-a_{-} \rho_{E}^{-}\right) .
\end{aligned}
$$

With the help of (2.4) and the requirement that $\left(\partial_{t}-c \Delta\right) \rho_{E}=0$ we obtain

$$
\begin{aligned}
F\left(v, \rho_{E}\right) & =\frac{\partial_{t} \rho_{E}}{1+\partial_{y} \rho_{E}} \partial_{y} v+c\left(\Delta_{\Theta}-\Delta\right) v \\
& =c\left(\frac{1+\left|\nabla_{x} \rho_{E}\right|^{2}}{\left(1+\partial_{y} \rho_{E}\right)^{2}}-1\right) \partial_{y}^{2} v-c \frac{2\left\langle\nabla_{x} \rho_{E} \mid \nabla_{x} \partial_{y} v\right\rangle}{1+\partial_{y} \rho_{E}} \\
- & \frac{c}{1+\partial_{y} \rho_{E}}\left\{\left(\frac{1+\left|\nabla_{x} \rho_{E}\right|^{2}}{\left(1+\partial_{y} \rho_{E}\right)^{2}}-1\right) \partial_{y}^{2} \rho_{E}-\frac{2\left\langle\nabla_{x} \rho_{E} \mid \nabla_{x} \partial_{y} \rho_{E}\right\rangle}{1+\partial_{y} \rho_{E}}\right\} \partial_{y} v .
\end{aligned}
$$

Using the fact that $\gamma \nabla_{x} v^{ \pm}=0$, a straightforward calculation also shows that

$$
H\left(v, \rho_{E}\right)=H_{+}\left(v, \rho_{E}\right)-H_{-}\left(v, \rho_{E}\right)
$$

with

$$
H_{ \pm}\left(v, \rho_{E}\right)=c_{ \pm}\left\{\left(1-\frac{1+\left|\gamma \nabla_{x} \rho_{E}^{ \pm}\right|^{2}}{1+\gamma \partial_{y} \rho_{E}^{ \pm}}\right) \gamma \partial_{y} v^{ \pm}-a_{ \pm} \gamma \partial_{y} \rho_{E}^{ \pm}\right\}
$$

By applying a fixed point argument we will prove in section 4 that under appropriate assumptions on the initial data, the coupled systems (2.5)-(2.6) admit a unique local-in-time solution $\left(v, \rho, \rho_{E}\right)$. This result will be based on a maximal regularity result for the linearized system (2.5), where the nonlinear functions $(F, H)$ are replaced by functions $(f, h)$ lying in suitable function spaces.

Remarks 2.1. (a) The additional term ' $a \rho_{E}$ ' appearing in the linearization of the Stefan condition in (2.5) is essential in our approach in order to get sufficient regularity for the function $\rho$ describing the free boundary, see also Remark 4.2 for additional information. Moreover, due to the fact that the function $\rho_{E}$ satisfies the equation $\left(\partial_{t}-c \Delta\right) \rho_{E}=0$, we do not have to consider the term

$$
\frac{\partial_{y} v}{1+\partial_{y} \rho_{E}}\left(\partial_{t} \rho_{E}-c \Delta \rho_{E}\right)
$$

which would otherwise occur in the first line of equation (2.5).

(b) We refer to $[6,7,8,9,10,11,12,13,33,45,49,52,53]$ for different results and approaches for the linearization of the classical two-phase Stefan problem. 
(c) It is interesting to note that the classical Stefan problem requires a finer analysis in order to establish maximal regularity than does the Stefan Problem with GibbsThomson correction, see [27]. This is, of course, not unexpected since surface tension is known to have a regularizing effect.

\section{Maximal Regularity For the Linearized PRoblem}

First let us introduce suitable function spaces. Let $\Omega \subseteq \mathbb{R}^{m}$ be open and $X$ be an arbitrary Banach space. By $L_{p}(\Omega ; X)$ and $H_{p}^{s}(\Omega ; X)$, for $1 \leq p \leq \infty, s \in$ $\mathbb{R}$, we denote the $X$-valued Lebegue and the Bessel potential space of order $s$, respectively. We will also frequently make use of the fractional Sobolev-Slobodeckij spaces $W_{p}^{s}(\Omega ; X), 1 \leq p<\infty, s \in \mathbb{R} \backslash \mathbb{Z}$, with norm

$$
\|g\|_{W_{p}^{s}(\Omega ; X)}=\|g\|_{W_{p}^{[s]}(\Omega ; X)}+\sum_{|\alpha|=[s]}\left(\int_{\Omega} \int_{\Omega} \frac{\left\|\partial^{\alpha} g(x)-\partial^{\alpha} g(y)\right\|_{X}^{p}}{|x-y|^{n+(s-[s]) p}} \mathrm{~d} x \mathrm{~d} y\right)^{1 / p}
$$

where $[s]$ denotes the largest integer smaller than $s$. Let $T \in(0, \infty]$ and $J=[0, T]$. We set

$$
{ }_{0} W_{p}^{s}(J ; X):=\left\{\begin{array}{l}
\left\{u \in W_{p}^{s}(J ; X): u(0)=u^{\prime}(0)=\ldots=u^{(k)}(0)=0\right\}, \\
\text { if } \quad k+\frac{1}{p}<s<k+1+\frac{1}{p}, k \in \mathbb{N} \cup\{0\}, \\
W_{p}^{s}(J ; X), \quad \text { if } \quad s<\frac{1}{p} .
\end{array}\right.
$$

The spaces ${ }_{0} H_{p}^{s}(J ; X)$ are defined analogously. Here we remind that $H_{p}^{k}=W_{p}^{k}$ for $k \in \mathbb{Z}$ and $1<p<\infty$, and that $W_{p}^{s}=B_{p p}^{s}$ for $s \in \mathbb{R} \backslash \mathbb{Z}$.

In this section we consider the linearized two-phase problem

$$
\left\{\begin{array}{rlll}
\left(\partial_{t}-c \Delta\right) v & = & & \text { in } J \times \dot{\mathbb{R}}^{n+1} \\
\gamma v^{ \pm} & =0 & & \text { on } J \times \mathbb{R}^{n} \\
\partial_{t} \rho+\left[c \gamma \partial_{y}\left(v-a \rho_{E}\right)\right] & = & & \text { on } J \times \mathbb{R}^{n} \\
v(0) & = & v_{0} & \text { in } \mathbb{R}^{n+1} \\
\rho(0) & = & \rho_{0} & \text { in } \mathbb{R}^{n}
\end{array}\right.
$$

with $c, a$ as defined in (2.3) and (2.7). We will always assume that the function $\rho_{E}$ satisfies equation (2.6), which we restate here for future reference

$$
\left\{\begin{aligned}
\left(\partial_{t}-c \Delta\right) \rho_{E} & =0 & & \text { in } J \times \dot{\mathbb{R}}^{n+1} \\
\gamma \rho_{E}^{ \pm} & =\rho & & \text { on } J \times \mathbb{R}^{n} \\
\rho_{E}(0) & =e^{-|y|\left(1-\Delta_{x}\right)^{\frac{1}{2}}} \rho_{0} & & \text { in } \dot{\mathbb{R}}^{n+1}
\end{aligned}\right.
$$

Remarks 3.1. (a) (3.2)-(3.3) constitutes a coupled system of equations, with the functions $\left(v, \rho, \rho_{E}\right)$ to be determined. We will in the sequel often just refer to a solution $(v, \rho)$ of $(3.2)$ with the understanding that the function $\rho_{E}$ also has to be determined. 
(b) Suppose $\rho \in W_{p}^{1-1 / 2 p}\left(J ; L_{p}\left(\mathbb{R}^{n}\right)\right) \cap L_{p}\left(J ; W_{p}^{2-1 / p}\left(\mathbb{R}^{n}\right)\right)$ and $\rho_{0} \in W_{p}^{2-3 / p}\left(\mathbb{R}^{n}\right)$ is given such that $\rho(0)=\rho_{0}$. Then the diffusion equation (3.3) admits a unique solution

$$
\rho_{E} \in W_{p}^{1}\left(J ; L^{p}\left(\dot{\mathbb{R}}^{n+1}\right)\right) \cap L_{p}\left(J ; W_{p}^{2}\left(\dot{\mathbb{R}}^{n+1}\right)\right) .
$$

This follows, for instance, from [27, Proposition 5.1], thanks to

$$
e^{-|y|\left(1-\Delta_{x}\right)^{\frac{1}{2}}} \rho_{0} \in W_{p}^{2-2 / p}\left(\dot{\mathbb{R}}^{n+1}\right) .
$$

(c) The solution $\rho_{E}(t, \cdot)$ of equation $(3.3)$ provides an extension of $\rho(t, \cdot)$ to $\dot{\mathbb{R}}^{n+1}$. We should remark that there are many possibilities to define such an extension. The chosen one is the most convenient one for our purposes. We also remark that we have great freedom for the extension of $\rho_{0}$.

We will first consider the special case that $\left(h(0), v_{0}, \rho_{0}\right)=(0,0,0)$. This allows us to derive an explicit representation for the solution of (3.2)-(3.3).

Proposition 3.2. Let $p \in(3, \infty), T>0$, and $J=[0, T]$. Suppose that $\left(h(0), v_{0}, \rho_{0}\right)=(0,0,0)$ and that

$$
\begin{aligned}
& f \in L_{p}\left(J ; L_{p}\left(\dot{\mathbb{R}}^{n+1}\right)\right) \text { and } \\
& h \in{ }_{0} W_{p}^{1 / 2-1 / 2 p}\left(J ; L_{p}\left(\mathbb{R}^{n}\right)\right) \cap L_{p}\left(J ; W_{p}^{1-1 / p}\left(\mathbb{R}^{n}\right)\right) .
\end{aligned}
$$

Then there is a unique solution $\left(v, \rho, \rho_{E}\right)$ of (3.2)-(3.3) satisfying

$$
\begin{aligned}
& v \in{ }_{0} W_{p}^{1}\left(J ; L^{p}\left(\dot{\mathbb{R}}^{n+1}\right)\right) \cap L_{p}\left(J ; W_{p}^{2}\left(\dot{\mathbb{R}}^{n+1}\right)\right), \\
& \rho \in{ }_{0} W_{p}^{3 / 2-1 / 2 p}\left(J ; L_{p}\left(\mathbb{R}^{n}\right)\right) \cap W_{p}^{1}\left(J ; W_{p}^{1-1 / p}\left(\mathbb{R}^{n}\right)\right) \cap L_{p}\left(J ; W_{p}^{2-1 / p}\left(\mathbb{R}^{n}\right)\right), \\
& \rho_{E} \in{ }_{0} W_{p}^{1}\left(J ; L^{p}\left(\dot{\mathbb{R}}^{n+1}\right)\right) \cap L_{p}\left(J ; W_{p}^{2}\left(\dot{\mathbb{R}}^{n+1}\right)\right) .
\end{aligned}
$$

The norm of the solution operator

$$
S_{T}:(f, h) \mapsto\left(v, \rho, \rho_{E}\right)
$$

is independent of the length of $J=[0, T]$ for any $T \leq T_{0}$, with $T_{0}$ arbitrary, but fixed.

Proof. (i) In order to be able to apply the Laplace transform in $t$, we consider the modified set of equations

$$
\left\{\begin{array}{rlrl}
\left(\partial_{t}+1-c \Delta\right) u & =f & \text { in }(0, \infty) \times \dot{\mathbb{R}}^{n+1} \\
\gamma u^{ \pm} & =0 \text { on }(0, \infty) \times \mathbb{R}^{n} \\
\left(\partial_{t}+1\right) \sigma+\left[c \gamma \partial_{y}\left(u-a \sigma_{E}\right)\right] & =h \quad & \text { on }(0, \infty) \times \mathbb{R}^{n} \\
u(0) & =0 & \text { in } \dot{\mathbb{R}}^{n+1} \\
\sigma(0) & =0 & \text { in } \mathbb{R}^{n}
\end{array}\right.
$$

and

$$
\left\{\begin{array}{rlll}
\left(\partial_{t}+1-c \Delta\right) \sigma_{E} & =0 & & \text { in }(0, \infty) \times \dot{\mathbb{R}}^{n+1} \\
\gamma \sigma_{E}^{ \pm} & =\sigma & & \text { on }(0, \infty) \times \mathbb{R}^{n} \\
\sigma_{E}(0) & =0 & & \text { in } \dot{\mathbb{R}}^{n+1}
\end{array}\right.
$$

for the unknown functions $\left(u, \sigma, \sigma_{E}\right)$. We claim that the system (3.5)-(3.6) admits a unique solution in the regularity class

$$
\begin{aligned}
& u \in{ }_{0} W_{p}^{1}\left(\mathbb{R}_{+} ; L^{p}\left(\dot{\mathbb{R}}^{n+1}\right)\right) \cap L_{p}\left(\mathbb{R}_{+} ; W_{p}^{2}\left(\dot{\mathbb{R}}^{n+1}\right)\right) \\
& \sigma \in{ }_{0} W_{p}^{3 / 2-1 / 2 p}\left(\mathbb{R}_{+} ; L_{p}\left(\mathbb{R}^{n}\right)\right) \cap{ }_{0} W_{p}^{1}\left(\mathbb{R}_{+} ; W_{p}^{1-1 / p}\left(\mathbb{R}^{n}\right)\right) \cap L_{p}\left(\mathbb{R}_{+} ; W_{p}^{2-1 / p}\left(\mathbb{R}^{n}\right)\right) \\
& \sigma_{E} \in{ }_{0} W_{p}^{1}\left(\mathbb{R}_{+} ; L^{p}\left(\dot{\mathbb{R}}^{n+1}\right)\right) \cap L_{p}\left(\mathbb{R}_{+} ; W_{p}^{2}\left(\dot{\mathbb{R}}^{n+1}\right)\right)
\end{aligned}
$$


for any functions $(f, h)$ with regularity

$$
(f, h) \in L_{p}\left(\mathbb{R}_{+} ; L_{p}\left(\dot{\mathbb{R}}^{n+1}\right)\right) \times\left({ }_{0} W_{p}^{1 / 2-1 / 2 p}\left(\mathbb{R}_{+} ; L_{p}\left(\mathbb{R}^{n}\right)\right) \cap L_{p}\left(\mathbb{R}_{+} ; W_{p}^{1-1 / p}\left(\mathbb{R}^{n}\right)\right)\right) .
$$

(ii) In the following, the symbol ^ denotes the Laplace transform w.r.t. $t$ combined with the Fourier transform w.r.t. the tangential space variable $x$. Applying the two transforms to equation (3.6) yields

$$
\left\{\begin{aligned}
\left(\omega^{2}-c \partial_{y}^{2}\right) \widehat{\sigma_{E}}(y) & =0, \quad y \in \dot{\mathbb{R}} \\
{\widehat{\sigma_{E}}}^{ \pm}(0) & =\hat{\sigma}
\end{aligned}\right.
$$

where we set

$$
\begin{aligned}
\omega & =\omega(\lambda, \xi, y)=\sqrt{\lambda+1+c(y)|\xi|^{2}}, \\
\omega_{ \pm} & =\omega_{ \pm}(\lambda, \xi)=\sqrt{\lambda+1+c_{ \pm}|\xi|^{2}} .
\end{aligned}
$$

Equation (3.7) can readily be solved to the result

$$
\widehat{\sigma_{E}}(y)=e^{-\frac{w}{\sqrt{c}}|y|} \hat{\sigma} .
$$

Next, applying the transforms to (3.5) we obtain

$$
\left\{\begin{aligned}
\left(\omega^{2}-c \partial_{y}^{2}\right) \hat{u}(y) & =\hat{f}(y), \quad y \in \dot{\mathbb{R}} \\
\hat{u}^{ \pm}(0) & =0 \\
(\lambda+1) \hat{\sigma}+\left[c \partial_{y}\left(\hat{u}-a \widehat{\sigma_{E}}\right)(0)\right] & =\hat{h} .
\end{aligned}\right.
$$

By employing the fundamental solution

$$
k_{ \pm}(y, s):=\frac{1}{2 \omega_{ \pm} \sqrt{c_{ \pm}}}\left(\mathrm{e}^{-\omega_{ \pm}|y-s| / \sqrt{c_{ \pm}}}-\mathrm{e}^{-\omega_{ \pm}(y+s) / \sqrt{c_{ \pm}}}\right), \quad y, s>0
$$

of the operator $\left(\omega_{ \pm}^{2}-c_{ \pm} \partial_{y}^{2}\right)$, we make for $\hat{u}^{ \pm}$the ansatz

$$
\begin{aligned}
& \hat{u}^{+}(y)=\int_{0}^{\infty} k_{+}(y, s) \hat{f}^{+}(s) \mathrm{d} s, \quad y>0, \\
& \hat{u}^{-}(y)=\int_{0}^{\infty} k_{-}(-y, s) \hat{f}^{-}(-s) \mathrm{d} s, \quad y<0 .
\end{aligned}
$$

A simple computation shows that

$$
\begin{aligned}
& \partial_{y} \hat{u}^{+}(0)=\frac{1}{c_{+}} \int_{0}^{\infty} \mathrm{e}^{-\omega_{+} s / \sqrt{c_{+}}} \hat{f}^{+}(s) \mathrm{d} s \quad \text { and } \\
& \partial_{y} \hat{u}^{-}(0)=-\frac{1}{c_{-}} \int_{0}^{\infty} \mathrm{e}^{-\omega_{-} s / \sqrt{c_{-}}} \hat{f}^{-}(-s) \mathrm{d} s .
\end{aligned}
$$

Inserting this and the fact that $\partial_{y}{\widehat{\sigma_{E}}}^{ \pm}(0)=\mp \frac{\omega_{ \pm}}{\sqrt{c_{ \pm}}} \hat{\sigma}$ in the third line of (3.9) gives

$$
\hat{\sigma}=\frac{1}{m}\left(\hat{h}-\int_{0}^{\infty} \mathrm{e}^{-\omega_{+} s / \sqrt{c_{+}}} \hat{f}^{+}(s) \mathrm{d} s-\int_{0}^{\infty} \mathrm{e}^{-\omega_{-} s / \sqrt{c_{-}}} \hat{f}^{-}(-s) \mathrm{d} s\right),
$$

with

$$
m=\lambda+1+a_{+} \sqrt{c_{+}} \omega_{+}+a_{-} \sqrt{c_{-}} \omega_{-} .
$$

(iii) In order to show the claimed regularity for the Laplace Fourier inverse of the 
representation $(\hat{u}, \hat{\sigma})$ let us first introduce some operators that correspond to the symbols occuring in (3.10)-(3.12). Let

$$
\mathcal{K} \in\{H, W\} .
$$

Then by $\mathcal{K}_{p}^{s}$ we either mean the space $H_{p}^{s}$ or the space $W_{p}^{s}$. Here and in what follows we make use of the notation and some of the results obtained in [27]. For instance, in exactly the same way as there it can be shown that the domain of the operator

$$
F_{ \pm}=\left(G+1+c_{ \pm} D_{n}\right)^{1 / 2} \quad \text { in }{ }_{0} H_{p}^{r}\left(\mathbb{R}_{+} ; \mathcal{K}_{p}^{s}\left(\mathbb{R}^{n}\right)\right)
$$

is given by

$$
\mathrm{D}\left(F_{ \pm}\right)={ }_{0} H_{p}^{r+1 / 2}\left(\mathbb{R}_{+} ; \mathcal{K}_{p}^{s}\left(\mathbb{R}^{n}\right)\right) \cap{ }_{0} H_{p}^{r}\left(\mathbb{R}_{+}, \mathcal{K}_{p}^{s+1}\left(\mathbb{R}^{n}\right)\right), \quad r, s \geq 0,
$$

and that $F_{ \pm}: \mathrm{D}\left(F_{ \pm}\right) \rightarrow{ }_{0} H_{p}^{r}\left(\mathbb{R}_{+} ; \mathcal{K}_{p}^{s}\left(\mathbb{R}^{n}\right)\right)$ is invertible. Here $G$ denotes the operator

$$
G u=\partial_{t} u, \quad u \in \mathrm{D}(G)={ }_{0} H_{p}^{r+1}\left(\mathbb{R}_{+} ; \mathcal{K}_{p}^{s}\left(\mathbb{R}^{n}\right)\right),
$$

and $D_{n}$ denotes the canonical extension to ${ }_{0} H_{p}^{r}\left(\mathbb{R}_{+} ; \mathcal{K}_{p}^{s}\left(\mathbb{R}^{n}\right)\right)$ of $-\Delta$ in $\mathcal{K}_{p}^{s}\left(\mathbb{R}^{n}\right)$, i.e.

$$
D_{n} u=-\Delta u \quad u \in \mathrm{D}\left(D_{n}\right)={ }_{0} H_{p}^{r}\left(\mathbb{R}_{+} ; \mathcal{K}_{p}^{s+2}\left(\mathbb{R}^{n}\right)\right) .
$$

According to the results in $[27$, pages $15-16]$,

$$
\begin{aligned}
& \int_{0}^{\infty} \mathrm{e}^{-F_{+} s / \sqrt{c_{+}}} f^{+}(s) \mathrm{d} s \in{ }_{0} W_{p}^{1 / 2-1 / 2 p}\left(\mathbb{R}_{+} ; L_{p}\left(\mathbb{R}^{n}\right)\right) \cap L_{p}\left(\mathbb{R}_{+} ; W_{p}^{1-1 / p}\left(\mathbb{R}^{n}\right)\right) \\
& \Longleftrightarrow f^{+} \in L_{p}\left(\mathbb{R}_{+} ; L_{p}\left(\mathbb{R}_{+}^{n+1}\right)\right) .
\end{aligned}
$$

And by the same arguments we have as well

$$
\begin{aligned}
& \int_{0}^{\infty} \mathrm{e}^{-F_{-} s / \sqrt{c_{-}}} f^{-}(-s) \mathrm{d} s \in{ }_{0} W_{p}^{1 / 2-1 / 2 p}\left(\mathbb{R}_{+} ; L_{p}\left(\mathbb{R}^{n}\right)\right) \cap L_{p}\left(\mathbb{R}_{+} ; W_{p}^{1-1 / p}\left(\mathbb{R}^{n}\right)\right) \\
& \Longleftrightarrow f^{-} \in L_{p}\left(\mathbb{R}_{+} ; L_{p}\left(\mathbb{R}_{-}^{n+1}\right)\right) .
\end{aligned}
$$

Next we show closedness and invertibility of the operator

$$
L:=G+1+a_{+} \sqrt{c_{+}} F_{+}+a_{-} \sqrt{c_{-}} F_{-},
$$

associated to the symbol $m$ introduced in $(3.12)$, in the space ${ }_{0} H_{p}^{r}\left(\mathbb{R}_{+}, \mathcal{K}_{p}^{s}\left(\mathbb{R}^{n}\right)\right)$. However, here we cannot directly apply the Dore-Venni result as it is done for the corresponding operator $L$ in [27]. This is due to the fact that we can a-priori not guarantee that the sum of the power angles of the single operators in $L$ is strictly less than $\pi$, which represents the limiting value in the Dore-Venni result. To our operator $L$ we apply a result of Kalton and Weis [36, Theorem 4.4], as demonstrated in the next lemma.

Lemma 3.3. Let $1<p<\infty, r, s \geq 0$, and $\mathcal{K} \in\{H, W\}$. Then

$$
\mathrm{D}(L)={ }_{0} H_{p}^{r+1}\left(\mathbb{R}_{+} ; \mathcal{K}_{p}^{s}\left(\mathbb{R}^{n}\right)\right) \cap{ }_{0} H_{p}^{r}\left(\mathbb{R}_{+} ; \mathcal{K}_{p}^{s+1}\left(\mathbb{R}^{n}\right)\right)
$$

and $L: \mathrm{D}(L) \rightarrow{ }_{0} H_{p}^{r}\left(\mathbb{R}_{+} ; \mathcal{K}_{p}^{s}\left(\mathbb{R}^{n}\right)\right)$ is invertible. 
Proof. Let $\varphi_{0} \in(0, \pi / 2)$ and $\varphi \in\left(0, \varphi_{0} / 2\right)$. We consider the function

$$
\begin{aligned}
f(\lambda, z, r) & :=\lambda+r+a_{+} \sqrt{c_{+}} \sqrt{r(\lambda+r)+c_{+} z^{2}}+a_{-\sqrt{c_{-}}} \sqrt{r(\lambda+r)+c_{-} z^{2}}, \\
(\lambda, z, r) & \in \bar{\Sigma}_{\pi-\varphi_{0}} \times \bar{\Sigma}_{\varphi} \times[0, \infty) \backslash\{(0,0,0)\} .
\end{aligned}
$$

Note that $\arg \lambda \geq 0$ implies that

$$
\arg \sqrt{r(\lambda+r)+c_{ \pm} z^{2}} \geq-\varphi, \quad(\lambda, z, r) \in \Sigma_{\pi-\varphi_{0}} \times \Sigma_{\varphi} \times[0, \infty) .
$$

In view of $\varphi<\varphi_{0} / 2$ this yields $f(\lambda, z, r) \neq 0$ on $\bar{\Sigma}_{\pi-\varphi_{0}} \times \bar{\Sigma}_{\varphi} \times[0, \infty) \backslash\{(0,0,0)\}$ for $\arg \lambda \geq 0$. On the other hand, we can argue in the same way if $\arg \lambda \leq$ 0 , which gives $f(\lambda, z, r) \neq 0$ on $\bar{\Sigma}_{\pi-\varphi_{0}} \times \bar{\Sigma}_{\varphi} \times[0, \infty) \backslash\{(0,0,0)\}$. Recall that by assumption $c_{+}, c_{-}, a_{+}, a_{-}$are stricly positive constants. This implies that the continuous function $|f|$ is stricly positive on the compact set

$$
\begin{aligned}
K:= & \left\{(\lambda, z, r) \in \bar{\Sigma}_{\pi-\varphi_{0}} \times \bar{\Sigma}_{\varphi} \times[0, \infty): R:=|\lambda|+r\right. \\
& \left.+a_{+} \sqrt{c_{+}}\left(\sqrt{r(|\lambda|+r)}+\sqrt{c_{+}}|z|\right)+a_{-} \sqrt{c_{-}}\left(\sqrt{r(|\lambda|+r)}+\sqrt{c_{-}}|z|\right)=1\right\} .
\end{aligned}
$$

Thus $|f| \geq c_{0}>0$ on $K$, which implies

$$
\begin{aligned}
|f(\lambda, z, r)|= & \left|f\left(\frac{\lambda}{R}, \frac{z}{R}, \frac{r}{R}\right)\right| \cdot R \\
\geq & c_{0}\left(|\lambda|+r+a_{+} \sqrt{c_{+}}\left(\sqrt{r(|\lambda|+r)}+\sqrt{c_{+}}|z|\right)\right. \\
& \left.+a_{-} \sqrt{c_{-}}\left(\sqrt{r(|\lambda|+r)}+\sqrt{c_{-}}|z|\right)\right)
\end{aligned}
$$

for all $(\lambda, z, r) \in \Sigma_{\pi-\varphi_{0}} \times \Sigma_{\varphi} \times(0, \infty)$ by virtue of $\left(\frac{\lambda}{R}, \frac{z}{R}, \frac{r}{R}\right) \in K$.

Now, observe that the symbol $m$ of the operator $L$ is given by $m=f(\cdot, \cdot, 1)$. The last estimate implies that the symbols

$$
m_{0}:=\frac{1}{m}, \quad m_{1}:=\frac{\lambda+1}{m}, \quad \text { and } \quad m_{ \pm}:=\frac{a_{ \pm} \sqrt{c_{ \pm}} \omega_{ \pm}}{m},
$$

are uniformly bounded for $(\lambda, z) \in \Sigma_{\pi-\varphi_{0}} \times \Sigma_{\varphi}$, where $\omega_{ \pm}(\lambda, z)=\sqrt{\lambda+1+c_{ \pm} z^{2}}$. Furthermore, note that we have

$$
D_{n}^{1 / 2} \in \mathcal{H}^{\infty}\left({ }_{0} H_{p}^{r}\left(\mathbb{R}_{+} ; \mathcal{K}_{p}^{s}\left(\mathbb{R}^{n}\right)\right)\right),
$$

that is, $D_{n}^{1 / 2}$ admits a bounded $\mathcal{H}^{\infty}$-calculus on ${ }_{0} H_{p}^{r}\left(\mathbb{R}_{+} ; \mathcal{K}_{p}^{s}\left(\mathbb{R}^{n}\right)\right)$, with $H^{\infty}$-angle $\phi_{D_{n}^{1 / 2}}^{\infty}=0$. (This follows, for instance, from Mikhlin's multiplier theorem.) Since the space ${ }_{0} H_{p}^{r}\left(\mathbb{R}_{+} ; \mathcal{K}_{p}^{s}\left(\mathbb{R}^{n}\right)\right)$ admits property $\alpha,[36$, Theorem 5.3] shows that we even have

$$
D_{n}^{1 / 2} \in \mathcal{R} \mathcal{H}^{\infty}\left({ }_{0} H_{p}^{r}\left(\mathbb{R}_{+} ; \mathcal{K}_{p}^{s}\left(\mathbb{R}^{n}\right)\right)\right)
$$

that is, $D_{n}^{1 / 2}$ admits an $\mathcal{R}$-bounded $\mathcal{H}^{\infty}$-calculus on ${ }_{0} H_{p}^{r}\left(\mathbb{R}_{+} ; \mathcal{K}_{p}^{s}\left(\mathbb{R}^{n}\right)\right)$, with $\mathcal{R} \mathcal{H}^{\infty}$ angle $\phi_{D_{n}^{1 / 2}}^{R \infty}=0$. By the uniform boundedness of $m_{j}, j \in\{0,1,+,-\}$, this implies that

$$
\mathcal{R}\left(\left\{m_{j}\left(\lambda, D_{n}^{1 / 2}\right): \lambda \in \Sigma_{\pi-\varphi_{0}}\right\}\right) \leq C, \quad j \in\{0,1,+,-\},
$$

where $\mathcal{R}(\mathcal{T})$ denotes the $\mathcal{R}$-bound of an operator family $\mathcal{T} \subseteq \mathcal{L}(X)$ for a Banach space $X$ (here $X={ }_{0} H_{p}^{r}\left(\mathbb{R}_{+} ; \mathcal{K}_{p}^{s}\left(\mathbb{R}^{n}\right)\right)$ ). Clearly, $m_{j}\left(\lambda, D_{n}^{1 / 2}\right)$ commutes with the resolvent of the operator $G$, and we know that $G \in \mathcal{H}^{\infty}\left({ }_{0} H_{p}^{r}\left(\mathbb{R}_{+} ; \mathcal{K}_{p}^{s}\left(\mathbb{R}^{n}\right)\right)\right)$ with 
$\phi_{G}^{\infty}=\pi / 2$, see [35]. In view of $\varphi_{0}<\pi / 2$ we may apply [36, Theorem 4.4] to the result

$$
m_{j}\left(G, D_{n}^{1 / 2}\right) \in \mathcal{L}\left({ }_{0} H_{p}^{r}\left(\mathbb{R}_{+} ; \mathcal{K}_{p}^{s}\left(\mathbb{R}^{n}\right)\right)\right), \quad j \in\{0,1,+,-\} .
$$

Now set $S:=m_{0}\left(G, D_{n}^{1 / 2}\right)$ and recall that

$$
G+1: \mathrm{D}(G) \rightarrow{ }_{0} H_{p}^{r}\left(\mathbb{R}_{+} ; \mathcal{K}_{p}^{s}\left(\mathbb{R}^{n}\right)\right), \quad F_{ \pm}: \mathrm{D}\left(F_{ \pm}\right) \rightarrow{ }_{0} H_{p}^{r}\left(\mathbb{R}_{+} ; \mathcal{K}_{p}^{s}\left(\mathbb{R}^{n}\right)\right)
$$

are invertible. By the uniqueness of the Fourier and Laplace transform this yields $(G+1)^{-1} m_{1}\left(G, D_{n}^{1 / 2}\right)=\left(a_{ \pm} \sqrt{c_{ \pm}} F_{ \pm}\right)^{-1} m_{ \pm}\left(G, D_{n}^{1 / 2}\right)=S \quad$ on $\quad{ }_{0} H_{p}^{r}\left(\mathbb{R}_{+} ; \mathcal{K}_{p}^{s}\left(\mathbb{R}^{n}\right)\right)$, and consequently

$$
S:{ }_{0} H_{p}^{r}\left(\mathbb{R}_{+} ; \mathcal{K}_{p}^{s}\left(\mathbb{R}^{n}\right)\right) \rightarrow \mathrm{D}(G) \cap \mathrm{D}\left(F_{ \pm}\right) .
$$

Again by the uniqueness of the Fourier and Laplace transform we conclude that $L S f=f$ for $f \in{ }_{0} H_{p}^{r}\left(\mathbb{R}_{+} ; \mathcal{K}_{p}^{s}\left(\mathbb{R}^{n}\right)\right)$ and $S L u=u$ for $u \in \mathrm{D}(G) \cap \mathrm{D}\left(F_{ \pm}\right)$. Thus, we have $S=L^{-1}$, i.e. $S$ is the bounded inverse of the operator $L$, which in particular implies that $L$ is closed in ${ }_{0} H_{p}^{r}\left(\mathbb{R}_{+} ; \mathcal{K}_{p}^{s}\left(\mathbb{R}^{n}\right)\right)$ and that

$$
\mathrm{D}(L)=\mathrm{D}(G) \cap \mathrm{D}\left(F_{ \pm}\right) .
$$

This proves the assertion in view of (3.13) and (3.14).

Next, we consider the formulas derived in (3.10), and (3.11), and start with the one for $u$.

(iv) Exemplary we will show the desired regularity for $u^{+}$. It is clear that we can establish the regularity for $u^{-}$in a similar way. Note that $u^{+}$is the solution of $\left(\partial_{t}+1-c_{+} \Delta\right) u^{+}=f$ in $\mathbb{R}_{+}^{n+1}$ with Dirichlet boundary conditions. Therefore it can be represented in terms of the solution operator $\left(G+1+c_{+} D_{n+1}\right)^{-1}$ in the whole space, namely as $u^{+}=P_{+}\left(G+1+c_{+} D_{n+1}\right)^{-1} E_{\text {odd }} f^{+}$, where $P_{+}: \mathbb{R}^{n+1} \rightarrow \mathbb{R}_{+}^{n+1}$ is the restriction operator and

$$
\left(E_{\text {odd }} f\right)(x, y):=\left\{\begin{array}{cc}
f(x, y), & y>0 \\
-f(x,-y), & y<0
\end{array}\right.
$$

is the extension by odd reflection. But then by

$$
P_{+} \in \mathcal{L}\left(L_{p}\left(\mathbb{R}_{+} ; W_{p}^{s}\left(\mathbb{R}^{n+1}\right)\right), L_{p}\left(\mathbb{R}_{+} ; W_{p}^{s}\left(\mathbb{R}_{+}^{n+1}\right)\right)\right),
$$

and

$$
E_{\text {odd }} \in \mathcal{L}\left(L_{p}\left(\mathbb{R}_{+} ; L_{p}\left(\mathbb{R}_{+}^{n+1}\right)\right), L_{p}\left(\mathbb{R}_{+} ; L_{p}\left(\mathbb{R}^{n+1}\right)\right)\right),
$$

for $u^{+}$the regularity in question is clear in view of the maximal regularity properties of the operator $\left(G+1+c_{+} D_{n+1}\right)^{-1}$.

(v) Observe that by (3.15) and (3.16) the terms $\int_{0}^{\infty} \mathrm{e}^{-F_{+} s / \sqrt{c_{+}}} f^{+}(s) \mathrm{d} s$ and $\int_{0}^{\infty} \mathrm{e}^{-F_{-} s / \sqrt{c_{-}}} f^{-}(-s) \mathrm{d} s$ belong to the regularity class of the data $h$. Therefore it remains to show that $L^{-1}$ maps the class of $h$ into the desired class for $\sigma$. Lemma 3.3 shows that

$$
L^{-1}: L_{p}\left(\mathbb{R}_{+} ; W_{p}^{1-1 / p}\left(\mathbb{R}^{n}\right)\right) \rightarrow{ }_{0} H_{p}^{1}\left(\mathbb{R}_{+} ; W_{p}^{1-1 / p}\left(\mathbb{R}^{n}\right)\right) \cap L_{p}\left(\mathbb{R}_{+} ; W_{p}^{2-1 / p}\left(\mathbb{R}^{n}\right)\right) .
$$

and moreover, that

$$
\begin{aligned}
& L^{-1}: L_{p}\left(\mathbb{R}_{+} ; L_{p}\left(\mathbb{R}^{n}\right)\right) \rightarrow{ }_{0} H_{p}^{1}\left(\mathbb{R}_{+} ; L_{p}\left(\mathbb{R}^{n}\right)\right) \cap L_{p}\left(\mathbb{R}_{+} ; H_{p}^{1}\left(\mathbb{R}^{n}\right)\right) \\
& L^{-1}:{ }_{0} W_{p}^{1}\left(\mathbb{R}_{+} ; L_{p}\left(\mathbb{R}^{n}\right)\right) \rightarrow{ }_{0} H_{p}^{2}\left(\mathbb{R}_{+} ; L_{p}\left(\mathbb{R}^{n}\right)\right) \cap{ }_{0} H_{p}^{1}\left(\mathbb{R}_{+} ; H_{p}^{1}\left(\mathbb{R}^{n}\right)\right) .
\end{aligned}
$$


By real interpolation we then obtain that $L^{-1}$ maps ${ }_{0} W_{p}^{1 / 2-1 / 2 p}\left(\mathbb{R}_{+} ; L_{p}\left(\mathbb{R}^{n}\right)\right)$ into

$$
{ }_{0} W_{p}^{3 / 2-1 / 2 p}\left(\mathbb{R}_{+} ; L_{p}\left(\mathbb{R}^{n}\right)\right) \cap{ }_{0} W_{p}^{1 / 2-1 / 2 p}\left(\mathbb{R}_{+} ; W_{p}^{1}\left(\mathbb{R}^{n}\right)\right) .
$$

Hence

$$
\begin{array}{r}
L^{-1} h \in{ }_{0} W_{p}^{3 / 2-1 / 2 p}\left(\mathbb{R}_{+} ; L_{p}\left(\mathbb{R}^{n}\right)\right) \cap{ }_{0} W_{p}^{1}\left(\mathbb{R}_{+} ; W_{p}^{1-1 / p}\left(\mathbb{R}^{n}\right)\right) \\
\cap{ }_{0} W_{p}^{1 / 2-1 / 2 p}\left(\mathbb{R}_{+} ; W_{p}^{1}\left(\mathbb{R}^{n}\right)\right) \cap L_{p}\left(\mathbb{R}_{+} ; W_{p}^{2-1 / p}\left(\mathbb{R}^{n}\right)\right),
\end{array}
$$

and the regularity assertion for $\sigma$ follows. Here we would like to remark that by Lemma 6.3, $\sigma$ has exactly the regularity claimed in Proposition 3.2.

(vi) By the trivial embedding

$$
{ }_{0} W_{p}^{3 / 2-1 / 2 p}\left(\mathbb{R}_{+} ; L_{p}\left(\mathbb{R}^{n}\right)\right) \hookrightarrow{ }_{0} W_{p}^{1-1 / 2 p}\left(\mathbb{R}_{+} ; L_{p}\left(\mathbb{R}^{n}\right)\right)
$$

and the just proved regularity for $\sigma$ we see that

$$
\sigma \in{ }_{0} W_{p}^{1-1 / 2 p}\left(\mathbb{R}_{+} ; L_{p}\left(\mathbb{R}^{n}\right)\right) \cap L_{p}\left(\mathbb{R}_{+} ; W_{p}^{2-1 / p}\left(\mathbb{R}^{n}\right)\right) .
$$

Remark 3.1(b) then implies the desired regularity for $\sigma_{E}$.

(vii) Let $I$ be either a finite interval $I:=[0, T]$, or $I:=\mathbb{R}_{+}$. Then we set

$$
{ }_{0} \mathbb{F}(I):=L_{p}\left(I ; L_{p}\left(\dot{\mathbb{R}}^{n+1}\right)\right) \times\left({ }_{0} W_{p}^{1 / 2-1 / 2 p}\left(I ; L_{p}\left(\mathbb{R}^{n}\right)\right) \cap L_{p}\left(I ; W_{p}^{1-1 / p}\left(\mathbb{R}^{n}\right)\right)\right)
$$

and

$$
\begin{aligned}
{ }_{0} \mathbb{Z}(I): & ={ }_{0} W_{p}^{1}\left(I ; L^{p}\left(\dot{\mathbb{R}}^{n+1}\right)\right) \cap L_{p}\left(I ; W_{p}^{2}\left(\dot{\mathbb{R}}^{n+1}\right)\right. \\
& \times\left({ }_{0} W_{p}^{3 / 2-1 / 2 p}\left(I ; L_{p}\left(\mathbb{R}^{n}\right)\right) \cap{ }_{0} W_{p}^{1}\left(I ; W_{p}^{1-1 / p}\left(\mathbb{R}^{n}\right)\right) \cap L_{p}\left(I ; W_{p}^{2-1 / p}\left(\mathbb{R}^{n}\right)\right)\right) \\
& \times\left({ }_{0} W_{p}^{1}\left(I ; L^{p}\left(\dot{\mathbb{R}}^{n+1}\right)\right) \cap L_{p}\left(I ; W_{p}^{2}\left(\dot{\mathbb{R}}^{n+1}\right)\right)\right) .
\end{aligned}
$$

Let $T_{0}>0$ be fixed, and let $J:=[0, T]$ with $T \leq T_{0}$. We set

$$
\begin{aligned}
\mathcal{R}_{J}^{c}:{ }_{0} \mathbb{F}(J) & \rightarrow{ }_{0} \mathbb{F}\left(\mathbb{R}_{+}\right) \\
(f, h) & \mapsto\left(e^{-t}\left(\mathcal{E}_{J} f\right), e^{-t}\left(\mathcal{E}_{J} g\right)\right),
\end{aligned}
$$

where $\mathcal{E}_{J}$ is defined in (6.2). It follows from Proposition 6.1 and the fact

$$
\left\|\left(e^{-t}\left(\mathcal{E}_{J} f\right), e^{-t}\left(\mathcal{E}_{J} g\right)\right)\right\|_{0 \mathbb{F}\left(\mathbb{R}_{+}\right)} \leq\left\|e^{-t}\right\|_{\mathrm{BUC}^{1}\left(\mathbb{R}_{+}\right)}\left\|\left(\mathcal{E}_{J} f, \mathcal{E}_{J} g\right)\right\|_{{ }_{0} \mathbb{F}\left(\mathbb{R}_{+}\right)}
$$

that there exists a positive constant $c_{0}=c_{0}\left(T_{0}\right)$ such that

$$
\left\|\mathcal{R}_{J}^{c}(f, h)\right\|_{0} \mathbb{F}\left(\mathbb{R}_{+}\right) \leq c_{0}\|(f, h)\|_{0} \mathbb{F}(J), \quad(f, h) \in{ }_{0} \mathbb{F}(J),
$$

for any interval $J=[0, T]$ with $T \leq T_{0}$.

Let $\left(u, \sigma, \sigma_{E}\right) \in{ }_{0} \mathbb{Z}\left(\mathbb{R}_{+}\right)$be the solution of (3.5)-(3.6), with $(f, h)$ replaced by $\left(\mathcal{R}_{J}^{c}(f, h)\right)$, whose existence has been established in steps (i)-(vi) of the proof. We note that

$$
\left\|\left(u, \sigma, \sigma_{E}\right)\right\|_{{ }_{o} \mathbb{Z}\left(\mathbb{R}_{+}\right)} \leq K\left\|\mathcal{R}_{J}^{c}(f, h)\right\|_{{ }_{0} \mathbb{F}\left(\mathbb{R}_{+}\right)} \leq K c_{0}\|(f, h)\|_{0 \mathbb{F}(J)}
$$

for any $(f, h) \in{ }_{0} \mathbb{F}(J)$ and and any interval $J=[0, T]$ with $T \leq T_{0}$, where $K$ is a universal constant. Finally, let

$$
\left(v, \rho, \rho_{E}\right):=\left(\mathcal{R}_{J}\left(e^{t} u\right), \mathcal{R}_{J}\left(e^{t} \sigma\right), \mathcal{R}_{J}\left(e^{t} \sigma_{E}\right)\right)
$$

where $\mathcal{R}_{J}$ denotes the restriction operator, defined by $\mathcal{R}_{J} w:=\left.w\right|_{J}$ for $w: \mathbb{R}_{+} \rightarrow X$. Then it is easy to verify that

$$
\left(v, \rho, \rho_{E}\right) \in{ }_{0} \mathbb{Z}(J), \quad\left(v, \rho, \rho_{E}\right) \text { solves }(3.2)-(3.3)
$$


and that there is a constant $M=M\left(T_{0}\right)$ such that

$$
\left\|\left(v, \rho, \rho_{E}\right)\right\|_{o \mathbb{Z}(J)} \leq M\|(f, h)\|_{0} \mathbb{F}(J)
$$

for any interval $J=[0, T]$ with $T \leq T_{0}$. Finally, uniqueness follows from the uniqueness of the Fourier and Laplace transform, and this completes the proof.

We are now ready to formulate our main result on the existence and uniqueness of a solution for (3.2)-(3.3). By introducing appropriate auxiliary functions, we will reduce this problem to the situation of Proposition 3.2.

Theorem 3.4. Let $p \in(3, \infty), T \in(0, \infty), J=[0, T]$.

(i) There exists a unique solution $\left(v, \rho, \rho_{E}\right)$ to $(3.2)-(3.3)$ with

$$
\begin{aligned}
& v \in W_{p}^{1}\left(J ; L^{p}\left(\dot{\mathbb{R}}^{n+1}\right)\right) \cap L_{p}\left(J ; W_{p}^{2}\left(\dot{\mathbb{R}}^{n+1}\right)\right), \\
& \rho \in W_{p}^{3 / 2-1 / 2 p}\left(J ; L_{p}\left(\mathbb{R}^{n}\right)\right) \cap W_{p}^{1}\left(J ; W_{p}^{1-1 / p}\left(\mathbb{R}^{n}\right)\right) \cap L_{p}\left(J ; W_{p}^{2-1 / p}\left(\mathbb{R}^{n}\right)\right), \\
& \rho_{E} \in W_{p}^{1}\left(J ; L^{p}\left(\dot{\mathbb{R}}^{n+1}\right)\right) \cap L_{p}\left(J ; W_{p}^{2}\left(\dot{\mathbb{R}}^{n+1}\right)\right),
\end{aligned}
$$

if and only if the data satisfy

$$
\begin{aligned}
& \text { (a) } f \in L_{p}\left(J ; L_{p}\left(\dot{\mathbb{R}}^{n+1}\right)\right), \\
& \text { (b) } h \in W_{p}^{1 / 2-1 / 2 p}\left(J ; L_{p}\left(\mathbb{R}^{n}\right)\right) \cap L_{p}\left(J ; W_{p}^{1-1 / p}\left(\mathbb{R}^{n}\right)\right), \\
& \text { (c) } v_{0} \in W_{p}^{2-2 / p}\left(\dot{\mathbb{R}}^{n+1}\right), \\
& \text { (d) } \rho_{0} \in W_{p}^{2-2 / p}\left(\mathbb{R}^{n}\right), \\
& \text { (e) } \gamma v_{0}=0 .
\end{aligned}
$$

(ii) If $\left(h(0), v_{0}, \rho_{0}\right)=(0,0,0)$, then the norm of the solution operator

$$
S_{T}:(f, h) \mapsto\left(v, \rho, \rho_{E}\right)
$$

is independent of the length of $J=[0, T]$ for any $T \leq T_{0}$, with $T_{0}$ arbitrary, but fixed.

Proof. It follows from the trace results in [27, Section 5] that the conditions listed in (a)-(e) are necessary.

Suppose we had a solution $\left(v, \rho, \rho_{E}\right)$ of $(3.2)-(3.3)$ as claimed in the statement of Theorem 3.4. Let $u_{1}$ be the solution of the two-phase diffusion equation

$$
\left\{\begin{array}{rlll}
\left(\partial_{t}-c \Delta\right) u_{1} & =0 & & \text { in } J \times \dot{\mathbb{R}}^{n+1}, \\
\gamma u_{1}^{ \pm} & =0 & & \text { on } J \times \mathbb{R}^{n}, \\
u_{1}(0) & =v_{0} & & \text { in } \dot{\mathbb{R}}^{n+1},
\end{array}\right.
$$

$\eta$ the extension function of Lemma 6.4(ii) with

$$
\left(\sigma_{0}, \sigma_{1}\right):=\left(\rho_{0}, h(0)-\left[c \gamma \partial_{y}\left(v_{0}-a e^{-|y|\left(1-\Delta_{x}\right)^{\frac{1}{2}}} \rho_{0}\right)\right]\right),
$$

and let $\eta_{E}$ be the solution of (3.3), with $\rho$ replaced by $\eta$. Then it follows from [27, Proposition 5.1], Lemma 6.4(ii), and Remark 3.1(b) that

$$
\begin{aligned}
& u_{1}, \eta_{E} \in W_{p}^{1}\left(J ; L^{p}\left(\dot{\mathbb{R}}^{n+1}\right)\right) \cap L_{p}\left(J ; W_{p}^{2}\left(\dot{\mathbb{R}}^{n+1}\right)\right) \\
& \eta \in W_{p}^{3 / 2-1 / 2 p}\left(J ; L_{p}\left(\mathbb{R}^{n}\right)\right) \cap W_{p}^{1}\left(J ; W_{p}^{1-1 / p}\left(\mathbb{R}^{n}\right)\right) \cap L_{p}\left(J ; W_{p}^{2-1 / p}\left(\mathbb{R}^{n}\right)\right) .
\end{aligned}
$$


One readily verifies that $\left(u, \sigma, \sigma_{E}\right):=\left(v, \rho, \rho_{E}\right)-\left(u_{1}, \eta, \eta_{E}\right)$ solves the linear problem

$$
\left\{\begin{aligned}
\left(\partial_{t}-c \Delta\right) u & =f & & \text { in } J \times \mathbb{R}^{n+1}, \\
\gamma u^{ \pm} & =0 & & \text { on } J \times \mathbb{R}^{n}, \\
\partial_{t} \sigma+\left[c \gamma \partial_{y}\left(u-a \sigma_{E}\right)\right] & =h-\partial_{t} \eta-\left[c \gamma \partial_{y}\left(u_{1}-a \eta_{E}\right)\right] & & \text { on } J \times \mathbb{R}^{n}, \\
u(0) & =0 & & \text { in } \dot{\mathbb{R}}^{n+1}, \\
\sigma(0) & =0 & & \text { in } \mathbb{R}^{n},
\end{aligned}\right.
$$

and

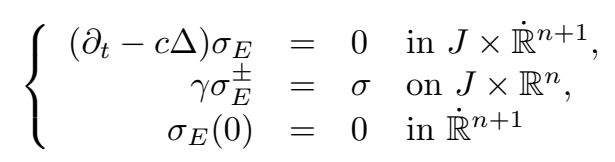

in the required regularity classes.

Reversing this argument we see that it suffices to consider the reduced system (3.21)-(3.22). To this end first observe that

$$
\left[\gamma \partial_{y}\left(u_{1}-a \eta_{E}\right)\right] \in W_{p}^{1 / 2-1 / 2 p}\left(J ; L_{p}\left(\mathbb{R}^{n}\right)\right) \cap L_{p}\left(J ; W_{p}^{1-1 / p}\left(\mathbb{R}^{n}\right)\right) .
$$

Thanks to the properties of $\eta$ we conclude that

$$
h-\partial_{t} \eta-\left[c \gamma \partial_{y}\left(u_{1}-a \eta_{E}\right)\right] \in{ }_{0} W_{p}^{1 / 2-1 / 2 p}\left(J ; L_{p}\left(\mathbb{R}^{n}\right)\right) \cap L_{p}\left(J ; W_{p}^{1-1 / p}\left(\mathbb{R}^{n}\right)\right) .
$$

Therefore we may apply Proposition 3.2, which yields the existence of a unique solution $\left(u, \sigma, \sigma_{E}\right)$ of $(3.21)-(3.22)$ in the desired regularity classes. It follows that

$$
\left(v, \rho, \rho_{E}\right)=\left(u, \sigma, \sigma_{E}\right)+\left(u_{1}, \eta, \eta_{E}\right)
$$

is a solution of (3.2)-(3.3) possessing the regularity properties claimed in the theorem. The uniqueness of the solution follows from Proposition 3.2, and the proof is now completed.

\section{The two-Phase Problem, local existence}

Recall that $\nabla_{x}$ and $\Delta_{x}$ denote the gradient and the Laplacian, respectively, with respect to $x$, whereas $\nabla$ and $\Delta$ denote the corresponding operators with respect to $(x, y) \in \mathbb{R}^{n} \times \mathbb{R}$.

From now on we assume $p>n+3$ and

$$
\left\|\nabla \rho_{E}\right\|_{\infty}=\left\|\nabla \rho_{E}\right\|_{L_{\infty}\left(J \times \mathbb{R}^{n+1}\right)} \leq 1 / 2,
$$

and consider the full two-phase Stefan problem in the transformed form (2.5). Here $\rho_{E}$ is the extension of

$$
\rho \in W_{p}^{3 / 2-1 / 2 p}\left(J ; L_{p}\left(\mathbb{R}^{n}\right)\right) \cap W_{p}^{1}\left(J ; W_{p}^{1-1 / p}\left(\mathbb{R}^{n}\right)\right) \cap L_{p}\left(J ; W_{p}^{2-1 / p}\left(\mathbb{R}^{n}\right)\right)
$$

satisfying equation (2.6). The above assumption is meaningful in view of

$$
\rho_{E} \in W_{p}^{1}\left(J ; L^{p}\left(\dot{\mathbb{R}}^{n+1}\right)\right) \cap L_{p}\left(J ; W_{p}^{2}\left(\dot{\mathbb{R}}^{n+1}\right)\right) \hookrightarrow \operatorname{BUC}\left(J ; W_{p}^{2-2 / p}\left(\dot{\mathbb{R}}^{n+1}\right)\right),
$$

see Proposition 6.2, and since, due to Sobolev's embedding theorem and $p>n+3$, the last space is continuously embedded in $\operatorname{BUC}\left(J ; \mathrm{BUC}^{1}\left(\dot{\mathbb{R}}^{n+1}\right)\right)$. Note that in space we applied the Sobolev embedding theorem separately on $\mathbb{R}_{+}^{n+1}$ and $\mathbb{R}_{-}^{n+1}$. 
We will frequently make use of the fact that for fixed $T_{0}>0$ there is a constant $c_{0}=c_{0}\left(T_{0}\right)$ such that

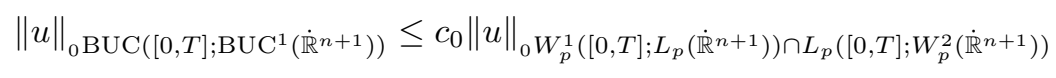

for all $u \in{ }_{0} W_{p}^{1}\left([0, T] ; L_{p}\left(\dot{\mathbb{R}}^{n+1}\right)\right) \cap L_{p}\left([0, T] ; W_{p}^{2}\left(\dot{\mathbb{R}}^{n+1}\right)\right)$ and all $T \in\left(0, T_{0}\right]$, see Proposition 6.2.

In the following we set $\mathbb{F}_{T}=\mathbb{F}_{T}^{1} \times \mathbb{F}_{T}^{2}$ for the regularity class of the data $(f, h)$, that is,

$$
\begin{aligned}
& \mathbb{F}_{T}^{1}:=L_{p}\left(J ; L_{p}\left(\dot{\mathbb{R}}^{n+1}\right)\right), \\
& \mathbb{F}_{T}^{2}:=W_{p}^{1 / 2-1 / 2 p}\left(J ; L_{p}\left(\mathbb{R}^{n}\right)\right) \cap L_{p}\left(J ; W_{p}^{1-1 / p}\left(\mathbb{R}^{n}\right)\right) .
\end{aligned}
$$

Moreover, we set $\mathbb{E}_{T}:=\mathbb{E}_{T}^{1} \times \mathbb{E}_{T}^{2}$ for the regularity class of $(v, \rho)$, that is,

$$
\begin{aligned}
& \mathbb{E}_{T}^{1}:=W_{p}^{1}\left(J ; L_{p}\left(\dot{\mathbb{R}}^{n+1}\right)\right) \cap L_{p}\left(J ; W_{p}^{2}\left(\dot{\mathbb{R}}^{n+1}\right)\right), \\
& \mathbb{E}_{T}^{2}:=W_{p}^{3 / 2-1 / 2 p}\left(J ; L_{p}\left(\mathbb{R}^{n}\right)\right) \cap W_{p}^{1}\left(J ; W_{p}^{1-1 / p}\left(\mathbb{R}^{n}\right)\right) \cap L_{p}\left(J ; W_{p}^{2-1 / p}\left(\mathbb{R}^{n}\right)\right),
\end{aligned}
$$

and $\mathbb{E}_{\gamma, T}:=\mathbb{E}_{\gamma, T}^{1} \times \mathbb{E}_{T}^{2}$ with

$$
\mathbb{E}_{\gamma, T}^{1}:=\left\{u \in \mathbb{E}_{T}^{1}: \gamma u^{+}=\gamma u^{-}=0\right\} .
$$

By ${ }_{0} \mathbb{E}_{T}:={ }_{0} \mathbb{E}_{T}^{1} \times{ }_{0} \mathbb{E}_{T}^{2},{ }_{0} \mathbb{E}_{\gamma, T}:={ }_{0} \mathbb{E}_{\gamma, T}^{1} \times{ }_{0} \mathbb{E}_{T}^{2}$, and ${ }_{0} \mathbb{F}_{T}^{2}$ we mean the corresponding spaces with zero time trace at $t=0$. Furthermore, we set for $b>0$

$$
\mathbb{E}_{T, b}^{1}:=\left\{w \in \mathbb{E}_{T}^{1}:\|\nabla w\|_{L_{\infty}\left(J \times \mathbb{R}^{n+1}\right)}<b\right\} .
$$

Clearly, $\mathbb{E}_{T, b}^{1}$ is an open subset of $\mathbb{E}_{T}^{1}$. Suppose $v, w \in \mathbb{E}_{T}$. Then we define

$$
\begin{aligned}
& \|\gamma D u\|_{\mathbb{F}_{T}}:=\left\|\gamma D u^{+}\right\|_{\mathbb{F}_{T}}+\left\|\gamma D u^{-}\right\|_{\mathbb{F}_{T}}, \\
& \|\gamma D u\|_{\infty}:=\left\|\gamma D u^{+}\right\|_{L_{\infty}\left(J \times \mathbb{R}^{n}\right)}+\left\|\gamma D u^{-}\right\|_{L_{\infty}\left(J \times \mathbb{R}^{n}\right)}, \quad u \in\{v, w\} \\
& \left\|\frac{\gamma \partial_{y} v}{1+\gamma \partial_{y} w}-a\right\|_{\infty}:=\left\|\frac{\gamma \partial_{y} v^{+}}{1+\gamma \partial_{y} w^{+}}-a_{+}\right\|_{L_{\infty}\left(J \times \mathbb{R}^{n}\right)}+\left\|\frac{\gamma \partial_{y} v^{-}}{1+\gamma \partial_{y} w^{-}}-a_{-}\right\|_{L_{\infty}\left(J \times \mathbb{R}^{n}\right)}
\end{aligned}
$$

where $D \in\left\{\partial_{j}, \partial_{y}, \nabla, \nabla_{x}\right\}$.

We will now list some properties for the nonlinear mappings $(F, H)$. We remind here that

$$
\begin{aligned}
F(v, w) & =c\left(\frac{1+\left|\nabla_{x} w\right|^{2}}{\left(1+\partial_{y} w\right)^{2}}-1\right) \partial_{y}^{2} v-c \frac{2\left\langle\nabla_{x} w \mid \nabla_{x} \partial_{y} v\right\rangle}{1+\partial_{y} w} \\
-c & \frac{\partial_{y} v}{1+\partial_{y} w}\left\{\left(\frac{1+\left|\nabla_{x} w\right|^{2}}{\left(1+\partial_{y} w\right)^{2}}-1\right) \partial_{y}^{2} w-\frac{2\left\langle\nabla_{x} w \mid \nabla_{x} \partial_{y} w\right\rangle}{1+\partial_{y} w}\right\}
\end{aligned}
$$

whereas $H$ was given by

$$
H(v, w)=H_{+}(v, w)-H_{-}(v, w)
$$

with

$$
H_{ \pm}(v, w)=c_{ \pm}\left\{\left(1-\frac{1+\left|\gamma \nabla_{x} w^{ \pm}\right|^{2}}{1+\gamma \partial_{y} w^{ \pm}}\right) \gamma \partial_{y} v^{ \pm}-a_{ \pm} \gamma \partial_{y} w^{ \pm}\right\}
$$


Proposition 4.1. Let $p>n+3$. Then we have

(a) $(F, H) \in C^{\omega}\left(\mathbb{E}_{T}^{1} \times \mathbb{E}_{T, 1 / 2}^{1}, \mathbb{F}_{T}^{1} \times \mathbb{F}_{T}^{2}\right)$.

(b) Let $D F(v, w)$ and $D H(v, w)$ denote the Fréchet derivatives of $F$ and $H$ at $(v, w) \in \mathbb{E}_{T}^{1} \times \mathbb{E}_{T, 1 / 2}^{1}$. Then

$$
(D F(v, w), D H(v, w)) \in \mathcal{L}\left({ }_{0} \mathbb{E}_{T}^{1} \times{ }_{0} \mathbb{E}_{T}^{1}, \mathbb{F}_{T}^{1} \times{ }_{0} \mathbb{F}_{T}^{2}\right),
$$

and there are constants $C_{1}=C_{1}\left(\alpha, T_{0}\right)$ and $C_{2}=C_{2}\left(\alpha, \beta, T_{0}\right)$ such that

$$
\|D F(v, w)\| \leq C_{1}\left(\|v\|_{\mathbb{E}_{T}^{1}}+\|w\|_{\mathbb{E}_{T}^{1}}+\|\nabla w\|_{\infty}\right)
$$

and

$$
\|D H(v, w)\| \leq C_{2}\left(\left\|\gamma \partial_{y} v\right\|_{\mathbb{F}_{T}^{2}}+\|\gamma \nabla w\|_{\mathbb{F}_{T}^{2}}+\|\gamma \nabla w\|_{\infty}+\left\|\frac{\gamma \partial_{y} v}{1+\gamma \partial_{y} w}-a\right\|_{\infty}\right)
$$

for all $(v, w) \in \mathbb{E}_{T}^{1} \times \mathbb{E}_{T, 1 / 2}^{1}$ with $\left\|\partial_{y} v\right\|_{L_{\infty}\left(J \times \dot{\mathbb{R}}^{n+1}\right)} \leq \alpha,\left\|\gamma \partial_{y} v\right\|_{\mathbb{F}_{T}^{2}} \leq \beta$ and all $T \leq T_{0}$. Here $\|D F(v, w)\|$ and $\|D H(v, w)\|$ denote the respective operator norms in the spaces indicated in (4.8).

Proof. In the following, we will repeatedly use the fact that multiplication

$$
L_{\infty}\left(J ; L_{\infty}\left(\dot{\mathbb{R}}^{n+1}\right)\right) \times L_{p}\left(J ; L_{p}\left(\dot{\mathbb{R}}^{n+1}\right)\right) \rightarrow L_{p}\left(J ; L_{p}\left(\dot{\mathbb{R}}^{n+1}\right)\right), \quad(f, g) \mapsto f g
$$

is continuous and bilinear (and hence also real analytic), with norm equal to one.

(a) We first note that $\|\nabla w\|_{L_{\infty}\left(J \times \mathbb{R}^{n+1}\right)} \leq 1 / 2$ implies

$$
\left\|\frac{1}{\left(1+\partial_{y} w\right)^{j}}\right\|_{L_{\infty}\left(J \times \mathbb{R}^{n+1}\right)} \leq 2^{j}, \quad j \in \mathbb{N} .
$$

Hence we deduce that

$$
\left(w \mapsto \frac{1}{\left(1+\partial_{y} w\right)^{k}}\right) \in C^{\omega}\left(\mathbb{E}_{T, 1 / 2}^{1}, L_{\infty}\left(J ; L_{\infty}\left(\dot{\mathbb{R}}^{n+1}\right)\right)\right)
$$

for $k=1,2$. From the representation (4.5) it is then easy to see that

$$
F \in C^{\omega}\left(\mathbb{E}_{T}^{1} \times \mathbb{E}_{T, 1 / 2}^{1}, \mathbb{F}_{T}^{1}\right) .
$$

Next, trace theory implies that

$$
\left((v, w) \mapsto\left(\gamma \partial v^{ \pm}, \gamma \partial w^{ \pm}\right)\right) \in \mathcal{L}\left(\mathbb{E}_{T}^{1} \times \mathbb{E}_{T}^{1}, \mathbb{F}_{T}^{2} \times \mathbb{F}_{T}^{2}\right)
$$

where $\partial$ stands either for $\partial_{j}, j=1, \ldots, n$, or $\partial_{y}$. Applying Lemma 6.6(ii),(vi) and (4.10) we may conclude that

$$
\left((v, w) \mapsto\left(1-\frac{1+\left|\gamma \nabla_{x} w^{ \pm}\right|^{2}}{1+\gamma \partial_{y} w^{ \pm}}\right) \gamma \partial_{y} v^{ \pm}\right) \in C^{\omega}\left(\mathbb{E}_{T}^{1} \times \mathbb{E}_{T, 1 / 2}^{1}, \mathbb{F}_{T}^{2}\right),
$$

and hence also that

$$
((v, w) \mapsto H(v, w)) \in C^{\omega}\left(\mathbb{E}_{T}^{1} \times \mathbb{E}_{T, 1 / 2}^{1}, \mathbb{F}_{T}^{2}\right) .
$$

For further use we note that

$$
\left\|\frac{1+\left|\nabla_{x} w\right|^{2}}{\left(1+\partial_{y} w\right)^{k}}-1\right\|_{\infty}=\left\|\frac{\left|\nabla_{x} w\right|^{2}-2(k-1) \partial_{y} w-\left(\partial_{y} w\right)^{k}}{\left(1+\partial_{y} w\right)^{k}}\right\|_{\infty} \leq C\|\nabla w\|_{\infty}
$$


for $w \in \mathbb{E}_{T, 1 / 2}^{1}$ and $k=1,2$. Also, we will frequently make use of the trivial fact that the norm of the trace operator $\gamma: \operatorname{BUC}\left(\dot{\mathbb{R}}^{n+1}\right) \rightarrow \mathrm{BUC}\left(\mathbb{R}^{n}\right)$ equals 1 , which implies

$$
\left\|\gamma \nabla w^{ \pm}\right\|_{L_{\infty}\left(J \times \mathbb{R}^{n}\right)} \leq\|\nabla w\|_{L_{\infty}\left(J \times \dot{\mathbb{R}}^{n+1}\right)} \leq 1 / 2,
$$

and therefore also that

$$
\left\|\frac{\gamma \nabla w^{ \pm}}{1+\gamma \partial_{y} w^{ \pm}}\right\|_{L_{\infty}\left(J \times \mathbb{R}^{n}\right)} \leq 1, \quad w \in \mathbb{E}_{T, 1 / 2}^{1}
$$

Moreover, it follows from the identity

$$
1-\frac{1+\left|\gamma \nabla_{x} w^{ \pm}\right|^{2}}{1+\gamma \partial_{y} w^{ \pm}}=\frac{\gamma \partial_{y} w^{ \pm}-\left|\gamma \nabla_{x} w^{ \pm}\right|^{2}}{1+\gamma \partial_{y} w^{ \pm}}
$$

and Lemma 6.6(iii),(v) that

$$
\left\|1-\frac{1+\left|\gamma \nabla_{x} w^{ \pm}\right|^{2}}{1+\gamma \partial_{y} w^{ \pm}}\right\|_{\mathbb{F}_{T}^{2}} \leq C\left\|\gamma \nabla w^{ \pm}\right\|_{\mathbb{F}_{T}^{2}}, \quad w \in \mathbb{E}_{T, 1 / 2}^{1} .
$$

(b) Let $(\bar{v}, \bar{w}) \in{ }_{0} \mathbb{E}_{T}^{1} \times{ }_{0} \mathbb{E}_{T}^{1}$ be given.

We will first consider the term

$$
F_{1}(v, w)=\left(\frac{1+\left|\nabla_{x} w\right|^{2}}{\left(1+\partial_{y} w\right)^{2}}-1\right) \partial_{y}^{2} v
$$

A straightforward computation shows that

$$
\begin{aligned}
D F_{1}(v, w)[\bar{v}, \bar{w}]= & \left(\frac{2\left\langle\nabla_{x} w \mid \nabla_{x} \bar{w}\right\rangle}{\left(1+\partial_{y} w\right)^{2}}-\frac{2\left(1+\left|\nabla_{x} w\right|^{2}\right) \partial_{y} \bar{w}}{\left(1+\partial_{y} w\right)^{3}}\right) \partial_{y}^{2} v \\
& +\left(\frac{1+\left|\nabla_{x} w\right|^{2}}{\left(1+\partial_{y} w\right)^{2}}-1\right) \partial_{y}^{2} \bar{v}
\end{aligned}
$$

Observing that all terms of $D F_{1}(v, w)[\bar{v}, \bar{w}]$ are made up of products of functions, with one factor always belonging to $\mathbb{E}_{T}^{1}$ and the remaining factors being in $\operatorname{BUC}(J \times$ $\dot{\mathbb{R}}^{n+1}$ ), and using in addition (4.9), (4.11), we readily obtain that

$$
\begin{aligned}
\left\|D F_{1}(v, w)[\bar{v}, \bar{w}]\right\|_{\mathbb{F}_{T}^{1}} & \leq C\left(\|v\|_{\mathbb{E}_{T}^{1}}+\|\nabla w\|_{\infty}\right)\left(\|\bar{v}\|_{0^{1}}+\|\nabla \bar{w}\|_{\infty}\right) \\
& \leq C\left(\|v\|_{\mathbb{E}_{T}^{1}}+\|\nabla w\|_{\infty}\right)\left(\|\bar{v}\|_{o_{0}}+\|\bar{w}\|_{0_{0} \mathbb{E}_{T}^{1}}\right)
\end{aligned}
$$

where $C$ is a universal constant for $(v, w) \in \mathbb{E}_{T}^{1} \times \mathbb{E}_{T, 1 / 2}^{1}$ and $(\bar{v}, \bar{w}) \in{ }_{0} \mathbb{E}_{T}^{1} \times{ }_{0} \mathbb{E}_{T}^{1}$ which is also independent of $T \in\left(0, T_{0}\right)$ for a fixed $T_{0}>0$. We remark that we used in the last step property (4.1). For the second term of $F, F_{2}(v, w)=$ $-2\left\langle\nabla_{x} w \mid \nabla_{x} \partial_{y} v\right\rangle /\left(1+\partial_{y} w\right)$, we obtain

$$
D F_{2}(v, w)[\bar{v}, \bar{w}]=-\frac{2\left\langle\nabla_{x} w \mid \nabla_{x} \partial_{y} \bar{v}\right\rangle+2\left\langle\nabla_{x} \bar{w} \mid \nabla_{x} \partial_{y} v\right\rangle}{1+\partial_{y} w}+\frac{2\left\langle\nabla_{x} w \mid \nabla_{x} \partial_{y} v\right\rangle \partial_{y} \bar{w}}{\left(1+\partial_{y} w\right)^{2}} .
$$

As above we conclude that

$$
\left\|D F_{2}(v, w)[\bar{v}, \bar{w}]\right\|_{\mathbb{F}_{T}^{1}} \leq C\left(\|v\|_{\mathbb{E}_{T}^{1}}+\|\nabla w\|_{\infty}\right)\left(\|\bar{v}\|_{0_{\mathbb{E}_{T}^{1}}}+\|\bar{w}\|_{0_{\mathbb{E}_{T}^{1}}}\right) .
$$

We consider now the last term

$$
F_{3}(v, w)=-\frac{\partial_{y} v}{1+\partial_{y} w}\left\{\left(\frac{1+\left|\nabla_{x} w\right|^{2}}{\left(1+\partial_{y} w\right)^{2}}-1\right) \partial_{y}^{2} w-\frac{\left.2\left\langle\nabla_{x} w \mid \nabla_{x} \partial_{y} w\right\rangle\right)}{1+\partial_{y} w}\right\}
$$


Here we observe that the expression in the curly brackets can be restated as $F_{1}(w, w)+F_{2}(w, w)$. Similar arguments as above then show the existence of a universal constant $C=C\left(\alpha, T_{0}\right)$ such that

$$
\left\|D F_{3}(v, w)[\bar{v}, \bar{w}]\right\|_{\mathbb{F}_{T}^{1}} \leq C\left(\|w\|_{\mathbb{E}_{T}^{1}}+\|\nabla w\|_{\infty}\right)\left(\|\bar{v}\|_{o_{0} \mathbb{E}_{T}^{1}}+\|\bar{w}\|_{o_{\mathbb{E}_{T}^{1}}^{1}}\right)
$$

whenever $(v, w)$ and $(\bar{v}, \bar{w})$ satisfy the assumptions of the proposition and $T \in\left(0, T_{0}\right)$. Summarizing all the estimates in (4.14) and (4.15)-(4.16) yields the estimate for $\|D F(v, w)\|$ asserted in the proposition.

We will now turn our attention to the nonlinear function $H(v, w)$, and we will focus on the term $H^{+}(v, w)$. Without fearing confusion, we will in the following just write $H$ instead of $H^{+}$, and $(v, w)$ instead of $\left(v^{+}, w^{+}\right)$. Moreover, we also set $a_{+}=a$ and $c_{+}=1$. A straightforward calculation shows that

$$
\begin{aligned}
D H(v, w)[\bar{v}, \bar{w}]= & \left(\frac{-2\left\langle\gamma \nabla_{x} w \mid \gamma \nabla_{x} \bar{w}\right\rangle}{1+\gamma \partial_{y} w}+\frac{\left|\gamma \nabla_{x} w\right|^{2}+1}{\left(1+\gamma \partial_{y} w\right)^{2}} \gamma \partial_{y} \bar{w}\right) \gamma \partial_{y} v \\
& +\left(1-\frac{1+\left|\gamma \nabla_{x} w\right|^{2}}{1+\gamma \partial_{y} w}\right) \gamma \partial_{y} \bar{v}-a \gamma \partial_{y} \bar{w} .
\end{aligned}
$$

We first observe that the derivative $D H(v, w)[\bar{v}, \bar{w}]$ is made up of products of functions, where one factor always has zero time trace at $t=0$. This implies that $D H(v, w)[\bar{v}, \bar{w}]$ lies in ${ }_{0} \mathbb{F}_{T}^{2}$.

The identity

$$
1 /\left(1+\gamma \partial_{y} w\right)^{2}=-\gamma \partial_{y} w /\left(1+\gamma \partial_{y} w\right)^{2}+1 /\left(1+\gamma \partial_{y} w\right)
$$

yields

$$
\begin{aligned}
D H(v, w)[\bar{v}, \bar{w}]= & \left(-\frac{2\left\langle\gamma \nabla_{x} w \mid \gamma \nabla_{x} \bar{w}\right\rangle}{1+\gamma \partial_{y} w}+\frac{\left|\gamma \nabla_{x} w\right|^{2}-\gamma \partial_{y} w}{\left(1+\gamma \partial_{y} w\right)^{2}} \gamma \partial_{y} \bar{w}\right) \gamma \partial_{y} v \\
& +\left(1-\frac{1+\left|\gamma \nabla_{x} w\right|^{2}}{1+\gamma \partial_{y} w}\right) \gamma \partial_{y} \bar{v}+\left(\frac{\gamma \partial_{y} v}{1+\gamma \partial_{y} w}-a\right) \gamma \partial_{y} \bar{w}
\end{aligned}
$$

By applying Lemma 6.6(iv) first for

$$
g=\gamma \partial_{y} v \quad \text { and } h=\frac{2 \gamma \partial_{j} w \gamma \partial_{j} \bar{w}}{1+\gamma \partial_{y} w}
$$

and then for

$$
g=\frac{\gamma \partial_{j} w}{1+\gamma \partial_{y} w}, \quad \text { and } h=\gamma \partial_{j} \bar{w}
$$

and afterwards using Lemma 6.6(v) in order to estimate $\left\|\frac{\gamma \partial_{j} w}{1+\gamma \partial_{y} w}\right\|_{\mathbb{F}_{T}^{2}}$, we obtain

$$
\begin{aligned}
\left\|\gamma \partial_{y} v \frac{\gamma \partial_{j} w \gamma \partial_{j} \bar{w}}{1+\gamma \partial_{y} w}\right\|_{\mathbb{F}_{T}^{2}} \leq & 2 c_{0}^{2}\left(\left\|\gamma \partial_{y} v\right\|_{\infty}+\left\|\gamma \partial_{y} v\right\|_{\mathbb{F}_{T}^{2}}\right) \\
& \cdot\left(\left\|\gamma \partial_{j} w\right\|_{\infty}+\left\|\gamma \partial_{j} w\right\|_{\mathbb{F}_{T}^{2}}+\left\|\gamma \partial_{y} w\right\|_{\mathbb{F}_{T}^{2}}\right)\left\|\gamma \partial_{j} \bar{w}\right\|_{0} \mathbb{F}_{T}^{2} \\
\leq & C\left(\|\gamma \nabla w\|_{\infty}+\|\gamma \nabla w\|_{\mathbb{F}_{T}^{2}}\right)\|\bar{w}\|_{0} \mathbb{E}_{T}^{1}
\end{aligned}
$$


with a universal constant $C$ for all $(v, w) \in \mathbb{E}_{T}^{1} \times \mathbb{E}_{T, 1 / 2}^{1}$ with $\left\|\gamma \partial_{y} v\right\|_{\infty} \leq \alpha$ and $\left\|\gamma \partial_{y} v\right\|_{\mathbb{F}_{T}^{2}} \leq \beta$, and all $T \leq T_{0}$. It follows that

$$
\left\|\gamma \partial_{y} v\left(\frac{-2\left\langle\gamma \nabla_{x} w \mid \gamma \nabla_{x} \bar{w}\right\rangle}{1+\gamma \partial_{y} w}\right)\right\|_{{ }_{0} \mathbb{F}_{T}^{2}} \leq C\left(\|\gamma \nabla w\|_{\infty}+\|\gamma \nabla w\|_{\mathbb{F}_{T}^{2}}\right)\|\bar{w}\|_{0} \mathbb{E}_{T}^{1} .
$$

A similar argument involving (4.12) also yields

$$
\left\|\gamma \partial_{y} v\left(\frac{\left|\gamma \nabla_{x} w\right|^{2}-\gamma \partial_{y} w}{\left(1+\gamma \partial_{y} w\right)^{2}} \gamma \partial_{y} \bar{w}\right)\right\|_{\mathbb{O}_{T}^{2}} \leq C\left(\|\gamma \nabla w\|_{\infty}+\|\gamma \nabla w\|_{\mathbb{F}_{T}^{2}}\right)\|\bar{w}\|_{0} \mathbb{E}_{T}^{1}
$$

It follows from Lemma 6.6(iv) and (4.11)-(4.13) that

$$
\begin{aligned}
\left\|\left(1-\frac{1+\left|\gamma \nabla_{x} w\right|^{2}}{1+\gamma \partial_{y} w}\right) \gamma \partial_{y} \bar{v}\right\|_{{ }_{0} \mathbb{F}_{T}^{2}} & \leq C\left(\|\gamma \nabla w\|_{\infty}+\|\gamma \nabla w\|_{\mathbb{F}_{T}^{2}}\right)\left\|\gamma \partial_{y} \bar{v}\right\|_{o_{\mathbb{F}_{T}^{2}}} \\
& \leq C\left(\|\gamma \nabla w\|_{\infty}+\|\gamma \nabla w\|_{\mathbb{F}_{T}^{2}}\right)\|\bar{v}\|_{o_{\mathbb{E}_{T}^{1}}^{1}}
\end{aligned}
$$

Finally, we conclude from Remark 6.7 and Lemma 6.6(v) that

$$
\begin{aligned}
& \left\|\left(\frac{\gamma \partial_{y} v}{1+\gamma \partial_{y} w}-a\right) \gamma \partial_{y} \bar{w}\right\|_{\mathbb{O}_{T}^{2}} \\
& \leq C\left(\left\|\frac{\gamma \partial_{y} v}{1+\gamma \partial_{y} w}-a\right\|_{\infty}+\left\|\gamma \partial_{y} v\right\|_{\mathbb{F}_{T}^{2}}+\left\|\gamma \partial_{y} w\right\|_{\mathbb{F}_{T}^{2}}\right)\|\bar{w}\|_{0 \mathbb{E}_{T}^{1}}
\end{aligned}
$$

Combining (4.17)-(4.20) yields the assertion for $\|D H(v, w)\|$, thus completing the proof of Proposition 4.1.

Remark 4.2. On the previous page we used the framed 1 in order to highlight where the second smallness condition of Theorem 1.1, or of Theorem 4.3, is used. First we remark that the term $\gamma \partial_{y} v /\left(1+\gamma \partial_{y} w\right)^{2}$ can only be made small under appropriate smallness assumptions on $v$, the temperature. However, the likely assumption that the uniform norm of $\gamma \partial_{y} v$ be small leads to an unnatural restriction for the initial temperature $v_{0}$. In contrast, the condition that the expression

$$
\left(\frac{\gamma \partial_{y} v}{1+\gamma \partial_{y} w}-a\right), \quad \text { where } \quad a=\frac{\gamma \partial_{y} v_{0}(0)}{1+\gamma \partial_{y} w_{0}(0)},
$$

be small follows from a smallness assumption on $\left(\frac{\gamma \partial_{y} v_{0}}{1+\gamma \partial_{y} w_{0}}-a\right)$, which can always be achieved for the Stefan problem by a judicious choice of a reference manifold and by a localization procedure (as will be shown somewhere else).

Having pointed out that subtracting the quantity $a$ from $\gamma \partial_{y} v /\left(1+\gamma \partial_{y} w\right)$ is advantageous in achieving smallness of the term in (4.21) - which is necessary for the fixed point argument - we now emphasize that the resulting effect of adding the number $a>0$ to the left side in $(2.5)-(2.6)$ is actually exactly the device that renders sufficient regularity for the linearized problem.

We are now ready for the main result of this section. 
Theorem 4.3. Fix $p>n+3$. Then there is a number $\eta>0$ such that the following holds: Given $\left(v_{0}, \rho_{0}\right) \in W_{p}^{2-2 / p}\left(\dot{\mathbb{R}}^{n+1}\right) \times W_{p}^{2-2 / p}\left(\mathbb{R}^{n}\right)$ with

$$
\gamma v_{0}^{ \pm}=0, \quad \pm v_{0}^{ \pm}>0 \text { on } \mathbb{R}_{ \pm}^{n+1}, \quad a_{ \pm}>0
$$

and

$$
\left\|\rho_{0}\right\|_{\mathrm{BUC}^{1}\left(\mathbb{R}^{n}\right)} \leq \eta, \quad\left\|\frac{\gamma \partial_{y} v_{0}^{ \pm}}{1+\gamma \partial_{y} w_{0}^{ \pm}}-a_{ \pm}\right\|_{B U C\left(\mathbb{R}^{n}\right)} \leq \eta / 2
$$

where

$$
w_{0}:=e^{-|y|\left(1-\Delta_{x}\right)^{\frac{1}{2}}} \rho_{0}, \quad a_{ \pm}:=\frac{\gamma \partial_{y} v_{0}^{ \pm}(0)}{1+\gamma \partial_{y} w_{0}^{ \pm}(0)},
$$

there exists $T=T\left(v_{0}, \rho_{0}\right)$ and a unique solution $(v, \rho) \in \mathbb{E}_{T}$ for $(2.5)-(2.6)$.

Remarks 4.4. (a) Note that $p>n+3$ implies that

$$
\rho_{0} \in W_{p}^{2-2 / p}\left(\mathbb{R}^{n}\right) \hookrightarrow \mathrm{BUC}^{1+\beta}\left(\mathbb{R}^{n}\right)
$$

for some $\beta>0$. Next, the interpolation inequality

$$
\|u\|_{\mathrm{BUC}^{1+\alpha}\left(\mathbb{R}^{n}\right)} \leq C(\alpha, \beta)\|u\|_{\mathrm{BUC}^{1+\beta}\left(\mathbb{R}^{n}\right)}^{\alpha / \beta}\|u\|_{\mathrm{BUC}^{1}\left(\mathbb{R}^{n}\right)}^{1-\alpha / \beta}, \quad u \in \mathrm{BUC}^{1+\beta}\left(\mathbb{R}^{n}\right),
$$

for $0<\alpha<\beta$ shows that there is an $\alpha>0$ such that

$$
\left\|\rho_{0}\right\|_{\mathrm{BUC}^{1+\alpha}\left(\mathbb{R}^{n}\right)} \leq C \eta \text {. }
$$

Thus, the conditions on $\rho_{0}$ imply that

$$
\left\|w_{0}\right\|_{\mathrm{BUC}^{1+\alpha}\left(\mathbb{R}^{n+1}\right)}=\left\|e^{-|y|\left(1-\Delta_{x}\right)^{\frac{1}{2}}} \rho_{0}\right\|_{\mathrm{BUC}^{1+\alpha}\left(\dot{\mathbb{R}}^{n+1}\right)} \leq c \eta
$$

for some constant $c=c\left(\left\|\rho_{0}\right\|_{W_{p}^{2-2 / p}\left(\mathbb{R}^{n}\right)}\right) \geq 1$. We will assume that $\eta$ is chosen small enough so that

$$
\left\|\nabla w_{0}\right\|_{L_{\infty}\left(\mathbb{R}^{n+1}\right)} \leq c \eta<1 / 8
$$

(b) It is clear that (4.25) and the conditions $\gamma v_{0}^{ \pm}=0$ and $\pm v_{0}^{ \pm}>0$ on $\mathbb{R}_{ \pm}^{n+1}$ already imply that $a_{ \pm} \geq 0$, but in general not that $a_{ \pm}>0$.

Proof. (i) It will be convenient to split the solution in a part with zero time trace at $t=0$ plus a remaining part taking care of the non-zero traces. For this purpose we employ Theorem 3.4, which gives us a solution $\left(v^{*}, \rho^{*}\right)$ for the linear problem (3.2) with given data

$$
\left(f, h, v_{0}, \rho_{0}\right)=\left(0, h^{*}, v_{0}, \rho_{0}\right) \quad \text { where } h^{*}(t):=\mathrm{e}^{t \Delta_{x}} H\left(v_{0}, w_{0}\right) .
$$

Note that the data in the line above satsify the assumptions (a)-(e) of Theorem 3.4, since for small $\left\|\nabla w_{0}\right\|_{\infty}$ we have $\left.H\left(v_{0}, w_{0}\right)=H(v, w)\right)\left.\right|_{t=0} \in W_{p}^{1-3 / p}\left(\mathbb{R}^{n}\right)$, and therefore

$$
h^{*} \in W_{p}^{1 / 2-1 / 2 p}\left(J ; L_{p}\left(\mathbb{R}^{n}\right)\right) \cap L_{p}\left(J ; W_{p}^{2-1 / p}\left(\mathbb{R}^{n}\right)\right) .
$$


Consequently, $\left(v^{*}, \rho^{*}\right) \in \mathbb{E}_{T}$ is well-defined and it suffices to study the reduced nonlinear problem

$$
\left\{\begin{aligned}
\left(\partial_{t}-c \Delta\right) \bar{v} & =F_{0}(\bar{v}, \bar{w}) & & \text { in } J \times \dot{\mathbb{R}}^{n+1} \\
\gamma \bar{v} & =0 & & \text { on } J \times \mathbb{R}^{n}, \\
\partial_{t} \bar{\rho}+\left[c \gamma \partial_{y}(\bar{v}-a \bar{w})\right] & =H_{0}(\bar{v}, \bar{w}) & & \text { on } J \times \mathbb{R}^{n}, \\
\bar{v}(0) & =0 & & \text { in } \mathbb{R}^{n+1} \\
\bar{\rho}(0) & =0 & & \text { in } \mathbb{R}^{n}
\end{aligned}\right.
$$

with

$$
F_{0}(\bar{v}, \bar{w}):=F\left(\bar{v}+v^{*}, \bar{w}+w^{*}\right), \quad H_{0}(\bar{v}, \bar{w}):=H\left(\bar{v}+v^{*}, \bar{w}+w^{*}\right)-h^{*},
$$

where

$\bar{w}$ and $w^{*}$ are extensions of $\bar{\rho}$ and $\rho^{*}$, respectively, satisfying (3.3).

Here we observe that $H_{0}(\bar{v}, \bar{w}) \in{ }_{0} \mathbb{F}_{T}^{2}$ for all functions $(\bar{v}, \bar{w}) \in{ }_{0} \mathbb{E}_{T}^{1} \times{ }_{0} \mathbb{E}_{T}^{1}$ with $w=\bar{w}+w^{*} \in \mathbb{E}_{T, 1 / 2}^{1}$. Thanks to this and Theorem 3.4(ii), the reduced nonlinear problem (4.26) can now be rephrased as a fixed point equation

$$
(\bar{v}, \bar{\rho})=K_{0}(\bar{v}, \bar{\rho}):=S_{T}\left(F_{0}(\bar{v}, \bar{w}), H_{0}(\bar{v}, \bar{w})\right) \text { in }{ }_{0} \mathbb{E}_{T},
$$

where $S_{T}$ is the solution operator of the linear problem defined in (3.20).

(ii) By applying the contraction mapping principle we will show the existence of a unique fixed point for equation (4.29). The advantage of applying the fixed point argument in the zero trace space ${ }_{0} \mathbb{E}_{T}$ lies in the fact that the embedding constant of the embedding

$$
{ }_{0} \mathbb{E}_{T} \hookrightarrow{ }_{0} \mathrm{BUC}\left(J ; \mathrm{BUC}^{1}\left(\dot{\mathbb{R}}^{n+1}\right)\right)
$$

does not depend on the length of the time interval $J=[0, T]$, i.e. there is a constant $c_{0}>0$ with

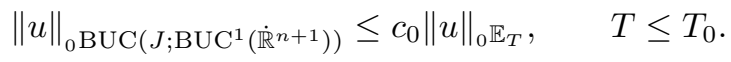

This enables us to choose $T$ as small as we wish for without having the embedding constant blowing up. Moreover, according to Theorem 3.4(ii), the norm of the solution operator $S_{T}$ is independent of $T$ as well, that is, there exists a number $M>0$ such that

$$
\left\|S_{T}\right\|_{\mathcal{L}\left(\mathbb{F}_{T}^{1} \times{ }_{0} \mathbb{F}_{T}^{2},{ }_{0} \mathbb{E}_{\gamma, T}^{1} \times{ }_{0} \mathbb{E}_{T}^{2}\right)} \leq M, \quad T \leq T_{0}
$$

(iii) In the following, we let ${ }_{0} \overline{\mathbb{B}}_{T}^{i}(0, r)$ be the closed ball of radius $r$ centered at 0 in ${ }_{0} \mathbb{E}_{T}^{i}$ with $i=1,2$. Moreover, we set

$$
\overline{\mathbb{B}}_{T}^{1}\left(v^{*}, r\right):=v^{*}+{ }_{0} \overline{\mathbb{B}}_{T}^{1}(0, r), \quad \overline{\mathbb{B}}_{T}^{1}\left(w^{*}, r\right):=w^{*}+{ }_{0} \overline{\mathbb{B}}_{T}^{1}(0, r) .
$$

Here we remark that $r$ and $T$ are independent parameters that can be chosen as we please. We first choose $r$ in such a way that

$$
\|\bar{v}\|_{{ }_{0} \mathbb{E}_{T}^{1}}+\left\|\gamma \partial_{y} \bar{v}\right\|_{o_{\mathbb{F}_{T}^{2}}}+\|\bar{w}\|_{{ }_{0} \mathbb{E}_{T}^{1}}+\|\gamma \nabla \bar{w}\|_{{ }_{0} \mathbb{F}_{T}^{2}}+\|\nabla \bar{w}\|_{0 \operatorname{BUC}\left(J ; \operatorname{BUC}\left(\dot{\mathbb{R}}^{n+1}\right)\right)} \leq c \eta
$$

for all $(\bar{v}, \bar{w}) \in{ }_{0} \overline{\mathbb{B}}_{T}^{1}(0, r) \times{ }_{0} \overline{\mathbb{B}}_{T}^{1}(0,4 N r)$, where $N$ is defined in (4.43). We also pick $T \in\left(0, T_{0}\right)$ small enough such that

$$
\left\|v^{*}\right\|_{\mathbb{E}_{T}^{1}}+\left\|\gamma \partial_{y} v^{*}\right\|_{\mathbb{F}_{T}^{2}}+\left\|w^{*}\right\|_{\mathbb{E}_{T}^{1}}+\left\|\gamma \nabla w^{*}\right\|_{\mathbb{F}_{T}^{2}}+\left\|\nabla w^{*}\right\|_{\mathrm{BUC}\left(J \times \dot{\mathbb{R}}^{n+1}\right)} \leq 3 c \eta .
$$


Indeed, by the fact that $w^{*}(t) \rightarrow w_{0}$ in $W_{p}^{2-2 / p}\left(\dot{\mathbb{R}}^{n+1}\right) \hookrightarrow \mathrm{BUC}^{1}\left(\dot{\mathbb{R}}^{n+1}\right)$ for $t \rightarrow 0$ and by (4.25) we obtain

$$
\left\|\nabla w^{*}\right\|_{\mathrm{BUC}\left(J ; \mathrm{BUC}\left(\dot{\mathbb{R}}^{n+1}\right)\right)} \leq 2 c \eta \quad \text { for small } T .
$$

Since $v^{*}$ and $w^{*}$ are fixed functions, all the remaining terms in (4.33) converge to 0 as $T \rightarrow 0$. We note that (4.25) and (4.32)-(4.33) imply

$$
\|\nabla w\|_{L_{\infty}\left(J \times \dot{\mathbb{R}}^{n+1}\right)}<1 / 2, \quad w \in \overline{\mathbb{B}}_{T}^{1}\left(w^{*}, 4 N r\right) .
$$

Thanks to (4.30) it is also clear that there is a number $\alpha>0$ such that

$$
\left\|\partial_{y} v\right\|_{L_{\infty}\left(\left(0, T_{0}\right) \times \mathbb{R}^{n+1}\right)} \leq \alpha, \quad v \in \overline{\mathbb{B}}_{T_{0}}^{1}\left(v^{*}, 1\right) .
$$

We can assume that the numbers $r$ and $T$ have been chosen small enough such that

$$
\left\|\frac{\gamma \partial_{y} v}{1+\gamma \partial_{y} w}-a\right\|_{\mathrm{BUC}\left(J \times \dot{\mathbb{R}}^{n+1}\right)} \leq 4 \eta, \quad(v, w) \in \overline{\mathbb{B}}_{T}^{1}\left(v^{*}, r\right) \times \overline{\mathbb{B}}_{T}^{1}\left(w^{*}, N r\right) .
$$

Indeed, an easy calculation shows that

$$
\begin{aligned}
\frac{\gamma \partial_{y} v(t)}{1+\gamma \partial_{y} w(t)}-a= & \frac{\gamma \partial_{y} \bar{v}(t)}{1+\gamma \partial_{y} w(t)}-\frac{\gamma \partial_{y} v^{*}(t) \gamma \partial_{y} \bar{w}(t)}{\left(1+\gamma \partial_{y} w(t)\right)\left(1+\gamma \partial_{y} w^{*}(t)\right)} \\
& +\left(\frac{\gamma \partial_{y} v^{*}(t)}{1+\gamma \partial_{y} w^{*}(t)}-a\right) .
\end{aligned}
$$

By (4.30) and (4.34)-(4.35) the first two terms can be made small by choosing $r$ small. Note that $v(t) \rightarrow v_{0}$ and $w(t) \rightarrow w_{0}$ in $W_{p}^{2-2 / p}\left(\dot{\mathbb{R}}^{n+1}\right) \hookrightarrow \mathrm{BUC}^{1}\left(\dot{\mathbb{R}}^{n+1}\right)$ for $t \rightarrow 0$, implying that also

$$
\frac{\gamma \partial_{y} v^{*}(t)}{1+\gamma \partial_{y} w^{*}(t)} \rightarrow \frac{\gamma \partial_{y} v_{0}}{1+\gamma \partial_{y} w_{0}} \quad \text { in } \mathrm{BUC}\left(\mathbb{R}^{n}\right) \text { for } t \rightarrow 0
$$

This and assumption (4.23) then yield the existence of a number $T$ such that

$$
\left\|\frac{\gamma \partial_{y} v^{*}(t)}{1+\gamma \partial_{y} w^{*}(t)}-a\right\|_{\infty} \leq 2 \eta, \quad 0 \leq t \leq T
$$

and the estimate in (4.36) follows. Combining (4.32)-(4.33) and (4.36) we obtain

$$
\begin{aligned}
\left(\|v\|_{\mathbb{E}_{T}^{1}}+\left\|\gamma \partial_{y} v\right\|_{\mathbb{F}_{T}^{2}}+\|w\|_{\mathbb{E}_{T}^{1}}+\right. & \|\gamma \nabla w\|_{\mathbb{F}_{T}^{2}}+\|\nabla w\|_{L_{\infty}\left(J \times \mathbb{R}^{n+1}\right)} \\
& \left.+\left\|\frac{\gamma \partial_{y} v}{1+\gamma \partial_{y} w}-a\right\|_{L_{\infty}\left(J \times \mathbb{R}^{n+1}\right)}\right) \leq 8 c \eta
\end{aligned}
$$

for all $(v, w) \in \overline{\mathbb{B}}_{T}^{1}\left(v^{*}, r\right) \times \overline{\mathbb{B}}_{T}^{1}\left(w^{*}, N r\right)$. We can now conclude from (4.35), (4.37), the definition of $\left(F_{0}, H_{0}\right)$, and Proposition 4.1 that

$$
\left\|D F_{0}(\bar{v}, \bar{w})\right\|+\left\|D H_{0}(\bar{v}, \bar{w})\right\| \leq K \eta, \quad(\bar{v}, \bar{w}) \in{ }_{0} \overline{\mathbb{B}}_{T}^{1}(0, r) \times{ }_{0} \overline{\mathbb{B}}_{T}^{1}(0, N r),
$$

where $K:=8 c \max \left\{C_{1}\left(\alpha, T_{0}\right), C_{2}\left(\alpha, 1, T_{0}\right)\right\}$. Moreover, we assume that $T$ was chosen so small that

$$
\left\|F_{0}(0,0)\right\|_{\mathbb{F}_{T}^{1}}+\left\|H_{0}(0,0)\right\|_{{ }_{0} \mathbb{F}_{T}^{2}} \leq r /(2 M),
$$

where $M$ is the constant given in (4.31). We will in the following assume that $r$ and $T$ have been fixed such that (4.38)-(4.39) holds with

$$
K \eta \leq \frac{1}{2 M(1+N)},
$$

where the constant $N$ is introduced in (4.43). 
(iv) Let $\left(\bar{v}_{i}, \bar{w}_{i}\right) \in{ }_{0} \overline{\mathbb{B}}_{T}^{1}(0, r) \times{ }_{0} \overline{\mathbb{B}}_{T}^{1}(0, N r)$ be given, where $i=1,2$. It follows from the mean value theorem and from $(4.38),(4.40)$ that

$$
\begin{aligned}
\|\left(F_{0}, H_{0}\right)\left(\bar{v}_{1}, \bar{w}_{1}\right)- & \left(F_{0}, H_{0}\right)\left(\bar{v}_{2}, \bar{w}_{2}\right) \|_{0} \mathbb{F}_{T} \\
& \leq \frac{1}{2 M(1+N)}\left\|\left(\bar{v}_{1}, \bar{w}_{1}\right)-\left(\bar{v}_{2}, \bar{w}_{2}\right)\right\|_{0 \mathbb{E}_{T}^{1} \times{ }_{0} \mathbb{E}_{T}^{1}} .
\end{aligned}
$$

In particular we obtain

$$
\left\|\left(F_{0}, H_{0}\right)(\bar{v}, \bar{w})-\left(F_{0}, H_{0}\right)(0,0)\right\|_{\mathbb{O}_{T}} \leq \frac{1}{2 M(1+N)}\|(\bar{v}, \bar{w})\|_{0 \mathbb{E}_{T}^{1} \times{ }_{0} \mathbb{E}_{T}^{1}}
$$

for all $(\bar{v}, \bar{w}) \in{ }_{0} \overline{\mathbb{B}}_{T}^{1}(0, r) \times{ }_{0} \overline{\mathbb{B}}_{T}^{1}(0, N r)$. This together with (4.39) yields

$$
\left\|\left(F_{0}, H_{0}\right)(\bar{v}, \bar{w})\right\|_{o_{\mathbb{F}}} \leq \frac{1}{2 M(1+N)}\|(\bar{v}, \bar{w})\|_{0} \mathbb{E}_{T}^{1} \times{ }_{0} \mathbb{E}_{T}^{1}+\frac{r}{2 M} \leq \frac{r}{M},
$$

for all $(\bar{v}, \bar{w}) \in{ }_{0} \overline{\mathbb{B}}_{T}^{1}(0, r) \times{ }_{0} \overline{\mathbb{B}}_{T}^{1}(0, N r)$.

(v) Given $\bar{\rho} \in{ }_{0} \mathbb{E}_{T}^{2}$, let $\bar{\rho}_{E} \in{ }_{0} \mathbb{E}_{T}^{1}$ be the extension of $\bar{\rho}$ satisfying (3.3). According to Proposition 3.2 there exists a constant $N>0$ such that

$$
\left\|\bar{\rho}_{E}\right\|_{{ }_{0} \mathbb{E}_{T}^{1}} \leq N\|\bar{\rho}\|_{0 \mathbb{E}_{T}^{2}}, \quad \bar{\rho} \in{ }_{0} \mathbb{E}_{T}^{2}, \quad T \leq T_{0},
$$

and it follows readily from (4.31) and (4.41)-(4.43) that $K_{0}=S_{T}\left(F_{0}, H_{0}\right)$ maps the set

$$
{ }_{0} \overline{\mathbb{B}}_{T}^{1}(0, r) \times{ }_{0} \overline{\mathbb{B}}_{T}^{2}(0, r) \subset{ }_{0} \mathbb{E}_{T}
$$

into itself, and that $K_{0}$ is a contraction. The assertion of the Theorem is now a consequence of the contraction mapping theorem.

\section{AnALYTicity}

Throughout this section $(v, \rho)$ denotes the unique solution to $(2.5)$ on $J=[0, T]$ with initial value $\left(v_{0}, \rho_{0}\right) \in W_{p}^{2-2 / p}\left(\dot{\mathbb{R}}^{n+1}\right) \times W_{p}^{2-2 / p}\left(\mathbb{R}^{n}\right)$. Recall that $\Gamma(t)=$ $\operatorname{graph}(\rho(t))$. Our goal is to show that

$$
M=\bigcup_{t \in(0, T)}(\{t\} \times \Gamma(t))
$$

is a real analytic manifold and that $(v, \rho)$ is in fact an analytic solution to (2.5). Again we will see that the property of maximal regularity for the linearized problem is of crucial importance.

Here the regularity classes are still denoted by $\mathbb{E}_{T}, \mathbb{F}_{T},{ }_{0} \mathbb{E}_{T}$, etc., i.e. we make use of the notation introduced in the previous section.

Given $\mu \in \mathbb{R}^{n}$ and $g \in \mathcal{S}\left(\mathbb{R}^{n}\right)$, the Schwartz space over $\mathbb{R}^{n}$, let $\tau_{\mu} g$ be the translation of $g$ by $\mu$, i.e.

$$
\left(\tau_{\mu} g\right)(x):=g(x+\mu), \quad x \in \mathbb{R}^{n} .
$$

It is not difficult to verify that $\tau_{\mu} \in \mathcal{L}\left(\mathcal{S}\left(\mathbb{R}^{n}\right)\right)$ and by duality, $\tau_{\mu}$ extends to a mapping, still denoted by $\tau_{\mu}$, such that $\tau_{\mu} \in \mathcal{L}\left(\mathcal{S}^{\prime}\left(\mathbb{R}^{n}\right)\right)$. We first prove the following result, cf. [24]. 
Lemma 5.1. Assume that

$$
X \in\left\{W_{p}^{s}\left(\mathbb{R}^{n}\right): s \in \mathbb{R}, p \in(1, \infty)\right\} .
$$

Then $\left\{\tau_{\mu}: \mu \in \mathbb{R}^{n}\right\}$ is a strongly continuous group of contractions on $X$, satisfying

$$
\tau_{\mu} g-\tau_{\mu_{0}} g=\int_{0}^{1} \tau_{\mu_{0}+s\left(\mu-\mu_{0}\right)}\left\langle\mu-\mu_{0} \mid \nabla g\right\rangle d s \quad \text { in } \quad X
$$

for any $g \in \mathcal{S}\left(\mathbb{R}^{n}\right)$.

Proof. If $X$ belongs to $\in\left\{W_{p}^{k}\left(\mathbb{R}^{n}\right): k \in \mathbb{Z}, p \in(1, \infty)\right\}$ the assertion follows from the transformation theorem for Lebesgue's integral and the mean value theorem. If $X$ belongs to the Slobodeckij scale $\left\{W_{p}^{s}\left(\mathbb{R}^{n}\right): s \in \mathbb{R} \backslash \mathbb{Z}, p \in(1, \infty)\right\}$ the assertion follows from the $W_{p}^{k}$-result by interpolation.

We need some further preparation. For this pick $T^{*} \in(0, T)$ and choose $\delta>0$ such that $\lambda t \in J$ for $\lambda \in(1-\delta, 1+\delta)$ and $t \in I:=\left[0, T^{*}\right]$. Given $g: I \rightarrow \mathcal{S}^{\prime}\left(\mathbb{R}^{n}\right)$ and $(\lambda, \mu) \in(1-\delta, 1+\delta) \times \mathbb{R}^{n}$, let

$$
g_{\lambda, \mu}(t):=\tau_{t \mu} g(\lambda t) \quad \text { for } t \in I .
$$

Then we have

Lemma 5.2. Given $h_{0} \in W_{p}^{1-3 / p}\left(\mathbb{R}^{n}\right)$ let $h^{*}(t):=e^{t \Delta_{x}} h_{0}$. Then there is a neighborhood $\Lambda$ of $(1,0)$ in $(1-\delta, 1+\delta) \times \mathbb{R}^{n}$ such that

$$
\left((\lambda, \mu) \mapsto h_{\lambda, \mu}^{*}\right) \in C^{\omega}\left(\Lambda, W_{p}^{1 / 2-1 / 2 p}\left(I ; L_{p}\left(\mathbb{R}^{n}\right)\right) \cap L_{p}\left(I ; W_{p}^{1-1 / p}\left(\mathbb{R}^{n}\right)\right) .\right.
$$

Proof. The proof of this result can be found in [27, pp. 39-40]. It is based on maximal regularity and the implicit function theorem.

As in the proof of Theorem 4.3 we set $h^{*}(t):=e^{t \Delta_{x}} H\left(v_{0}, w_{0}\right), t \in J$, and we denote by $\left(v^{*}, \rho^{*}, \rho_{E}^{*}\right) \in \mathbb{E}_{T} \times \mathbb{E}_{T}^{1}$ the unique solution of

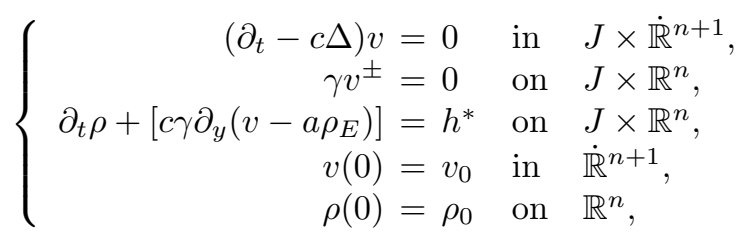

with $\rho_{E}^{*}$ the extension of $\rho^{*}$ satisfying equation (3.3) with $\rho$ replaced by $\rho^{*}$.

Given $\mu \in \mathbb{R}^{n}$ and $f \in L_{p}\left(\dot{\mathbb{R}}^{n+1}\right)$, let $\tau_{\mu} f$ be defined by

$$
\left(\tau_{\mu} f\right)(x, y):=f(x+\mu, y), \quad(x, y) \in \dot{\mathbb{R}}^{n+1} .
$$

We note that the functions

$$
v_{\lambda, \mu}^{*}(t):=\tau_{t \mu} v^{*}(\lambda t), \quad \rho_{\lambda, \mu}^{*}(t):=\tau_{t \mu} \rho^{*}(\lambda t) \quad \text { and } \quad w_{\lambda, \mu}^{*}:=\tau_{t \mu} \rho_{E}^{*}(\lambda t)
$$

are well-defined for all $(\lambda, \mu) \in(1-\delta, 1+\delta) \times \mathbb{R}^{n}$ and all $t \in I$. 
Proposition 5.3. There exists a neighborhood $\Lambda \subset(1-\delta, 1+\delta) \times \mathbb{R}^{n}$ of $(1,0)$ such that

$$
\left[(\lambda, \mu) \mapsto\left(v_{\lambda, \mu}^{*}, \rho_{\lambda, \mu}^{*}, w_{\lambda, \mu}^{*}\right)\right] \in C^{\omega}\left(\Lambda, \mathbb{E}_{T^{*}} \times \mathbb{E}_{T^{*}}^{1}\right)
$$

Proof. (i) Let $\Lambda \subset(1-\delta, 1+\delta) \times \mathbb{R}^{n}$ be the open neighborhood of $(1,0)$ obtained in Lemma 5.2. We will first show that

$$
\left(v_{\lambda, \mu}^{*}, \rho_{\lambda, \mu}^{*}, w_{\lambda, \mu}^{*}\right) \in \mathbb{E}_{T^{*}} \times \mathbb{E}_{T^{*}}^{1}, \quad(\lambda, \mu) \in \Lambda .
$$

Let $(\lambda, \mu) \in \Lambda$ be fixed. As in the proof of [27, Lemma 8.2] one verifies that

$$
u_{\lambda, \mu}^{*} \in W_{p}^{1}\left(I ; L_{p}\left(\dot{\mathbb{R}}^{n+1}\right)\right) \cap L_{p}\left(I ; W_{p}^{2}\left(\dot{\mathbb{R}}^{n+1}\right)\right), \quad u \in\{v, w\},
$$

and

$$
\frac{d}{d t} u_{\lambda, \mu}^{*}(t)=\lambda \tau_{t \mu} \frac{d}{d t} u^{*}(\lambda t)+\left\langle\mu \mid \nabla_{x} u_{\lambda, \mu}^{*}(t)\right\rangle, \quad u \in\{v, w\}
$$

in $L_{p}\left(\dot{\mathbb{R}}^{n+1}\right)$ a.e. on $I$.

Moreover, we also have

$$
\rho_{\lambda, \mu}^{*} \in W_{p}^{1}\left(I ; W_{p}^{1-1 / p}\left(\mathbb{R}^{n}\right)\right) \cap L_{p}\left(I ; W_{p}^{2-1 / p}\left(\mathbb{R}^{n}\right)\right),
$$

and

$$
\frac{d}{d t} \rho_{\lambda, \mu}^{*}(t)=\lambda \tau_{t \mu} \frac{d}{d t} \rho^{*}(\lambda t)+\left\langle\mu \mid \nabla_{x} \rho_{\lambda, \mu}^{*}(t)\right\rangle
$$

a.e. on $I$.

In order to verify that $\rho_{\lambda, \mu}^{*} \in W_{p}^{3 / 2-1 / 2 p}\left(I ; L_{p}\left(\mathbb{R}^{n}\right)\right)$ it suffices to show that

$$
\frac{d}{d t} \rho_{\lambda, \mu}^{*} \in W_{p}^{1 / 2-1 / 2 p}\left(I ; L_{p}\left(\mathbb{R}^{n}\right)\right) .
$$

Equation (5.5) and Lemma 6.3 yield $\rho_{\lambda, \mu}^{*} \in W_{p}^{1 / 2-1 / 2 p}\left(I ; W_{p}^{1}\left(\mathbb{R}^{n}\right)\right)$. Thus, thanks to (5.6), it remains to show that

$$
\left(t \mapsto \tau_{t \mu} \dot{\rho}^{*}(\lambda t)\right) \in W_{p}^{1 / 2-1 / 2 p}\left(I ; L_{p}\left(\mathbb{R}^{n}\right)\right),
$$

where we set $\dot{\rho}^{*}:=\frac{d}{d t} \rho^{*}$. In order to do so we use, as in section 4 , the intrinsic norm

$$
\|g\|_{W_{p}^{1 / 2-1 / 2 p}\left(I ; L_{p}\left(\mathbb{R}^{n}\right)\right)}=\|g\|_{L_{p}\left(I ; L_{p}\left(\mathbb{R}^{n}\right)\right)}+\left(\int_{I} \int_{I} \frac{\|g(t)-g(s)\|_{L_{p}\left(\mathbb{R}^{n}\right)}^{p}}{|t-s|^{p / 2+1 / 2}} \mathrm{~d} \mathrm{~d} t\right)^{1 / p} .
$$

We have

$$
\begin{aligned}
\left\|\dot{\rho}_{\lambda, \mu}^{*}(t)-\dot{\rho}_{\lambda, \mu}^{*}(s)\right\|_{L_{p}}^{p} & \leq C\left(\left\|\tau_{t \mu}\left(\dot{\rho}^{*}(\lambda t)-\dot{\rho}^{*}(\lambda s)\right)\right\|_{L_{p}}^{p}+\left\|\left(\tau_{t \mu}-\tau_{s \mu}\right) \dot{\rho}^{*}(\lambda s)\right\|_{L_{p}}^{p}\right) \\
& \leq C\left(\left\|\dot{\rho}^{*}(\lambda t)-\dot{\rho}^{*}(\lambda s)\right\|_{L_{p}}^{p}+\left\|\left(\tau_{t \mu}-\tau_{s \mu}\right) \dot{\rho}^{*}(\lambda s)\right\|_{L_{p}}^{p}\right),
\end{aligned}
$$

and, recalling that $\dot{\rho}^{*} \in W_{p}^{1 / 2-1 / 2 p}\left(I ; L_{p}\left(\mathbb{R}^{n}\right)\right)$, we readily conclude

$$
\int_{I} \int_{I} \frac{\left\|\dot{\rho}^{*}(\lambda t)-\dot{\rho}^{*}(\lambda s)\right\|_{L_{p}\left(\mathbb{R}^{n}\right)}^{p}}{|t-s|^{p / 2+1 / 2}} d s d t<\infty
$$

Using Lemma 5.1 and interpolation theory we obtain

$$
\left\|\left(\tau_{t \mu}-\tau_{s \mu}\right) \dot{\rho}^{*}(\lambda s)\right\|_{L_{p}\left(\mathbb{R}^{n}\right)} \leq C|t-s|^{(1-1 / p)}\left\|\dot{\rho}^{*}(\lambda s)\right\|_{W_{p}^{1-1 / p}\left(\mathbb{R}^{n}\right)}
$$


for allmost all $s \in I$. Thus

$$
\begin{aligned}
\int_{I} \int_{I} \frac{\left\|\left(\tau_{t \mu}-\tau_{s \mu}\right) \dot{\rho}^{*}(\lambda s)\right\|_{L_{p}}^{p}}{|t-s|^{p / 2+1 / 2} \mathrm{~d} s} \mathrm{~d} t & \left.\leq C \int_{I} \int_{I}|t-s|^{(p / 2-3 / 2)} \| \dot{\rho}^{*}(\lambda s)\right) \|_{W_{p}^{1-1 / p}}^{p} \mathrm{~d} s \mathrm{~d} t \\
& \left.\leq C \int_{I} \| \dot{\rho}^{*}(\lambda s)\right) \|_{W_{p}^{1-1 / p}\left(\mathbb{R}^{n}\right)}^{p} \mathrm{~d} s<\infty .
\end{aligned}
$$

Combining all the steps we arrive at (5.3).

(ii) Next we observe that $\tau_{\mu_{0}}(\Delta f)=\Delta\left(\tau_{\mu_{0}} f\right)$ and $\tau_{\mu_{0}}\left[\partial_{y} f\right]=\left[\partial_{y}\left(\tau_{\mu_{0}} f\right)\right]$ for any $\mu_{0} \in \mathbb{R}^{n}$ and $f \in W_{p}^{2}\left(\dot{\mathbb{R}}^{n+1}\right)$. Combining these facts with (5.4) and (5.6), we conclude that $(u, \sigma, w)=\left(v_{\lambda, \mu}^{*}, \rho_{\lambda, \mu}^{*}, w_{\lambda, \mu}^{*}\right)$ is a solution of

$$
\left\{\begin{aligned}
\left(\partial_{t}-\lambda c \Delta\right) u & =\left\langle\mu \mid \nabla_{x} u\right\rangle & & \text { in } I \times \dot{\mathbb{R}}^{n+1}, \\
\gamma u^{ \pm} & =0 & & \text { on } I \times \mathbb{R}^{n}, \\
\partial_{t} \sigma+\lambda\left[c \gamma \partial_{y}(u-a w)\right] & =\lambda h_{\lambda, \mu}^{*}+\left\langle\mu \mid \nabla_{x} \sigma\right\rangle & & \text { on } I \times \mathbb{R}^{n}, \\
u(0) & =v_{0} & & \text { in } \mathbb{R}^{n+1}, \\
\sigma(0) & =\rho_{0} & & \text { on } \mathbb{R}^{n}
\end{aligned}\right.
$$

and

$$
\left\{\begin{aligned}
\left(\partial_{t}-\lambda c \Delta\right) w & =\left\langle\mu \mid \nabla_{x} w\right\rangle & & \text { in } I \times \dot{\mathbb{R}}^{n+1} \\
\gamma w^{ \pm} & =\sigma & & \text { on } I \times \mathbb{R}^{n} \\
w(0) & =e^{-|y|\left(1-\Delta_{x}\right)^{\frac{1}{2}}} \sigma(0) & & \text { in } \quad \dot{\mathbb{R}}^{n+1}
\end{aligned}\right.
$$

(iii) Given $(\sigma, \lambda, \mu) \in \mathbb{E}_{T^{*}}^{2} \times \Lambda$, let $w=\mathcal{T}(\sigma, \lambda, \mu)$ be the solution of (5.8). Then one shows that

$$
\begin{aligned}
& {[(\sigma, \lambda, \mu) \mapsto \mathcal{T}(\sigma, \lambda, \mu)] \in C^{\omega}\left(\mathbb{E}_{T^{*}}^{2} \times \Lambda, \mathbb{E}_{T^{*}}^{1}\right),} \\
& D_{1} \mathcal{T}(\sigma, 1,0)[\tilde{\sigma}]=\tilde{\sigma}_{E},
\end{aligned}
$$

where, as usual, $\tilde{\sigma}_{E}$ is the extension of $\tilde{\sigma}$ satisfying (3.3), and where $D_{1} \mathcal{T}$ denotes the derivative of $\mathcal{T}$ with respect to $\sigma$. The proof can, for instance, be based on maximal regularity and the implicit function theorem.

We now define the mapping

$$
\begin{aligned}
\Psi: \mathbb{E}_{\gamma, T^{*}} \times \Lambda \rightarrow \mathbb{G}_{T^{*}}:=\mathbb{F}_{T^{*}} \times W_{p}^{2-2 / p}\left(\dot{\mathbb{R}}^{n+1}\right) \times W_{p}^{2-2 / p}\left(\mathbb{R}^{n}\right) \\
\Psi((u, \sigma),(\lambda, \mu)):=\left(\begin{array}{l}
\left(\partial_{t}-\lambda c \Delta\right) u-\left\langle\mu \mid \nabla_{x} u\right\rangle \\
\partial_{t} \sigma+\lambda\left[c \gamma \partial_{y}(u-a \mathcal{T}(\sigma, \lambda, \mu))\right]-\lambda h_{\lambda, \mu}^{*}-\left\langle\mu \mid \nabla_{x} \sigma\right\rangle \\
u(0)-v_{0} \\
\sigma(0)-\rho_{0}
\end{array}\right) .
\end{aligned}
$$

It follows from (the sufficient part of) Theorem 3.4 and from Lemma 6.3 that $\Psi$ is well-defined. Moreover, Lemma 5.2 and (5.9) imply that $\Psi$ is analytic. Further, writing $D_{1} \Psi$ for the derivative of $\Psi$ with respect to $(u, \sigma)$ we find

$$
D_{1} \Psi\left(\left(v^{*}, \rho^{*}\right),(1,0)\right)[\tilde{u}, \tilde{\sigma}]=\left(\left(\partial_{t}-c \Delta\right) \tilde{u}, \partial_{t} \tilde{\sigma}+\left[c \gamma \partial_{y}\left(\tilde{u}-a \tilde{\sigma}_{E}\right)\right], \tilde{u}(0), \tilde{\sigma}(0)\right) .
$$

Since $D_{1} \Psi\left(\left(v^{*}, \rho^{*}\right),(1,0)\right) \in \mathcal{L}\left(\mathbb{E}_{\gamma, T^{*}}, \mathbb{G}_{T^{*}}\right)$ we infer from Theorem 3.4 and the open mapping theorem that

$$
D_{1} \Psi\left(\left(v^{*}, \rho^{*}\right),(1,0)\right) \in \operatorname{Isom}\left(\mathbb{E}_{\gamma, T^{*}}, \mathbb{G}_{T^{*}}\right) .
$$

Since $\Psi((u, \sigma),(\lambda, \mu))=0$ holds true if, and only if, $(u, \sigma)$ is a solution in $\mathbb{E}_{T^{*}}$ to (5.7), the implicit function theorem gives the assertion for $\left(v_{\lambda, \mu}^{*}, \rho_{\lambda, \mu}^{*}\right)$ for an open 
neighborhood of $(1,0)$ in $\Lambda$, which we will again denote by $\Lambda$. Finally we observe that $w_{\lambda, \mu}^{*}=\mathcal{T}\left(\rho_{\lambda, \mu}^{*}, \lambda, \mu\right)$, and it follows from $(5.9)$ that $\left[(\lambda, \mu) \mapsto w_{\lambda, \mu}^{*}\right]$ is analytic as well.

Theorem 5.4. There exists an open neighborhood $\Lambda$ of $(1,0)$ in $(1-\delta, 1+\delta) \times \mathbb{R}^{n}$ such that

$$
\left[(\lambda, \mu) \mapsto\left(v_{\lambda, \mu}, \rho_{\lambda, \mu}, w_{\lambda, \mu}\right)\right] \in C^{\omega}\left(\Lambda, \mathbb{E}_{T^{*}} \times \mathbb{E}_{T^{*}}^{1}\right)
$$

where $(v, \rho)$ is the solution of $(2.5)$, and $w=\rho_{E}$.

Proof. (i) It follows from the proof of Theorem 4.3 that the solution $(v, \rho)$ of $(2.5)$ admits the representation

$$
(v, \rho)=(\bar{v}, \bar{\rho})+\left(v^{*}, \rho^{*}\right) \quad \text { with } \quad(\bar{v}, \bar{\rho}) \in{ }_{0} \overline{\mathbb{B}}_{T}^{1}(0, r) \times{ }_{0} \overline{\mathbb{B}}_{T}^{2}(0, r),
$$

where $(\bar{v}, \bar{\rho})$ is the solution of $(4.26)$ and ${ }_{0} \overline{\mathbb{B}}_{T}^{j}(0, r) \subseteq{ }_{0} \mathbb{E}_{T}^{j}, j=1,2$, denotes, as before, the closed ball with radius $r$ and center 0 . Using Lemma 5.1 it is not difficult to verify that there is an open neighborhood $\Lambda$ of $(1,0)$ in $(1-\delta, 1+\delta) \times \mathbb{R}^{n}$ such that

$$
\left(\bar{v}_{\lambda, \mu}, \bar{\rho}_{\lambda, \mu}\right) \in{ }_{0} \mathbb{B}_{T^{*}}^{1}(0,2 r) \times{ }_{0} \mathbb{B}_{T^{*}}^{2}(0,2 r), \quad(\lambda, \mu) \in \Lambda .
$$

Let $w=\mathcal{T}(\sigma, \lambda, \mu)$ be the solution of

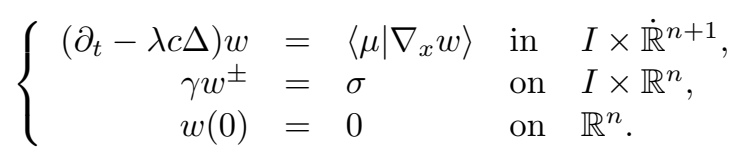

As in Proposition 5.3, one verifies that

$$
\begin{aligned}
& {[(\sigma, \lambda, \mu) \mapsto \mathcal{T}(\sigma . \lambda, \mu)] \in C^{\omega}\left({ }_{0} \mathbb{E}_{T^{*}}^{2} \times \Lambda,{ }_{0} \mathbb{E}_{T^{*}}^{1}\right),} \\
& D_{1} \mathcal{T}(\sigma, 1,0)[\tilde{\sigma}]=\tilde{\sigma}_{E} .
\end{aligned}
$$

Moreover, for a suitable neighborhood $\Lambda \subseteq(1-\delta, 1+\delta) \times \mathbb{R}^{n}$ we have

$$
\|\mathcal{T}(\sigma, \lambda, \mu)\|_{\mathbb{E}_{T^{*}}^{1}} \leq 2 N\|\sigma\|_{\mathbb{E}_{T^{*}}^{2}}, \quad \sigma \in{ }_{0} \mathbb{E}_{T^{*}}^{2}, \quad(\lambda, \mu) \in \Lambda
$$

where $N$ is the constant introduced in (4.43). Due to (5.11) and (4.34) we may assume, by possibly making $\Lambda$ smaller, that

$$
\left(u+v_{\lambda, \mu}^{*}, \mathcal{T}(\sigma, \lambda, \mu)+w_{\lambda, \mu}^{*}\right) \in \mathbb{E}_{\gamma, T^{*}}^{1} \times \mathbb{E}_{T^{*}, 1 / 2}^{1}
$$

for all $(\lambda, \mu) \in \Lambda$ and all $(u, \sigma) \in{ }_{0} \mathbb{B}_{\gamma, T^{*}}^{1}(0,2 r) \times{ }_{0} \mathbb{B}_{T^{*}}^{2}(0,2 r)$, where $v_{\lambda, \mu}^{*}$ and $w_{\lambda, \mu}^{*}$ are defined in (5.2).

Given $(\lambda, \mu) \in \Lambda$ and $(u, \sigma) \in{ }_{0} \mathbb{B}_{T^{*}}^{1}(0,2 r) \times{ }_{0} \mathbb{B}_{T^{*}}^{2}(0,2 r)$ we set

$$
\begin{aligned}
F_{\lambda, \mu}(u, \sigma) & :=\lambda F\left(u+v_{\lambda, \mu}^{*}, \mathcal{T}(\sigma, \lambda, \mu)+w_{\lambda, \mu}^{*}\right)+\left\langle\mu \mid \nabla_{x} u\right\rangle, \\
H_{\lambda, \mu}(u, \sigma) & :=\lambda H\left(u+v_{\lambda, \mu}^{*}, \mathcal{T}(\sigma, \lambda, \mu)+w_{\lambda, \mu}^{*}\right)-\lambda h_{\lambda, \mu}^{*}+\left\langle\mu \mid \nabla_{x} \sigma\right\rangle .
\end{aligned}
$$

It follows from (5.10), (5.12), Proposition 4.1(a), Lemma 5.2, and Proposition 5.3 that

$$
\begin{aligned}
& {\left[((u, \sigma),(\lambda, \mu)) \mapsto\left(F_{\lambda, \mu}(u, \sigma), H_{\lambda, \mu}(u, \sigma)\right)\right]} \\
& \quad \in C^{\omega}\left({ }_{0} \mathbb{B}_{T^{*}}^{1}(0,2 r) \times{ }_{0} \mathbb{B}_{T^{*}}^{2}(0,2 r) \times \Lambda,{ }_{0} \mathbb{F}_{T^{*}}\right) .
\end{aligned}
$$

(ii) As in the proof of Proposition 5.3 one shows that $(u, \sigma, w)=\left(\bar{v}_{\lambda, \mu}, \bar{\rho}_{\lambda, \mu}, \bar{w}_{\lambda, \mu}\right)$ 
is a solution of

$$
\left\{\begin{aligned}
\left(\partial_{t}-\lambda c \Delta\right) u & =F_{\lambda, \mu}(u, \sigma) & & \text { in } I \times \dot{\mathbb{R}}^{n+1} \\
\gamma u^{ \pm} & =0 & & \text { on } I \times \mathbb{R}^{n} \\
\partial_{t} \sigma+\lambda\left[c \gamma \partial_{y}(u-a w)\right] & =H_{\lambda, \mu}(u, \sigma) & & \text { on } I \times \mathbb{R}^{n} \\
u(0) & =0 & & \text { in } \mathbb{R}^{n+1} \\
\sigma(0) & =0 & & \text { on } \mathbb{R}^{n}
\end{aligned}\right.
$$

and

$$
\left\{\begin{aligned}
\left(\partial_{t}-\lambda c \Delta\right) w & =\left\langle\mu \mid \nabla_{x} w\right\rangle & & \text { in } I \times \dot{\mathbb{R}}^{n+1} \\
\gamma w^{ \pm} & =\sigma & & \text { on } I \times \mathbb{R}^{n}, \\
w(0) & =0 & & \text { on } \mathbb{R}^{n} .
\end{aligned}\right.
$$

Indeed, the assertion follows from equation (4.26) since $\Delta,\left[c \gamma \partial_{y}\right]$ and the nonlinear mappings $(F, H)$ commute with translations, i.e. we have

$$
\begin{aligned}
& \tau_{\mu} \Delta v=\Delta \tau_{\mu} v, \quad \tau_{\mu}\left[c \gamma \partial_{y}(v-a w)\right]=\left[c \gamma \partial_{y} \tau_{\mu}(v-a w)\right], \\
& \tau_{\mu} F(v, w)=F\left(\tau_{\mu} v, \tau_{\mu} w\right), \quad \tau_{\mu} H(v, w)=H\left(\tau_{\mu} v, \tau_{\mu} w\right),
\end{aligned}
$$

for $(v, w)$ and $\mu$ suitable.

(iii) Next, we introducde the nonlinear mapping

$$
\begin{aligned}
& \Psi_{0}:{ }_{0} \mathbb{B}_{\gamma, T^{*}}^{1}(0,2 r) \times{ }_{0} \mathbb{B}_{T^{*}}^{2}(0,2 r) \times \Lambda \rightarrow{ }_{0} \mathbb{F}_{T^{*}} \\
& \Psi_{0}((u, \sigma),(\lambda, \mu)):=\left(\begin{array}{l}
\left(\partial_{t}-\lambda c \Delta\right) u-F_{\lambda, \mu}(u, \sigma) \\
\partial_{t} \sigma+\lambda\left[c \gamma \partial_{y}(u-a \mathcal{T}(\sigma, \lambda, \mu))\right]-H_{\lambda, \mu}(u, \sigma)
\end{array}\right) .
\end{aligned}
$$

We infer from $\left(v_{\lambda, \mu}^{*}(0), w_{\lambda, \mu}^{*}(0)\right)=\left(v_{0}, w_{0}\right),\left(u(0), \sigma(0), \partial_{t} \sigma(0)\right)=(0,0,0)$ and the definition of $h^{*}$ that $\Psi_{0}((u, \sigma),(\lambda, \mu)) \in{ }_{0} \mathbb{F}_{T^{*}}$, and this shows that $\Psi_{0}$ is welldefined. It follows from (5.10) and (5.13) that

$$
\Psi_{0} \in C^{\omega}\left({ }_{0} \mathbb{B}_{\gamma, T^{*}}^{1}(0,2 r) \times{ }_{0} \mathbb{B}_{T^{*}}^{2}(0,2 r) \times \Lambda,{ }_{0} \mathbb{F}_{T^{*}}\right) .
$$

Next, writing $D_{1} \Psi_{0}$ for the derivative of $\Psi_{0}$ with respect to $(u, \sigma)$ we find

$$
D_{1} \Psi_{0}((\bar{v}, \bar{\rho}),(1,0))[\tilde{u}, \tilde{\sigma}]=U[\tilde{u}, \tilde{\sigma}]-\left(D F_{0}\left(\bar{v}, \bar{\rho}_{E}\right), D H_{0}\left(\bar{v}, \bar{\rho}_{E}\right)\right)\left[\tilde{u}, \tilde{\sigma}_{E}\right]
$$

for $(\tilde{u}, \tilde{\sigma}) \in{ }_{0} \mathbb{E}_{\gamma, T^{*}}$, where

$$
U[\tilde{u}, \tilde{\sigma}]:=\left(\left(\partial_{t}-c \Delta\right) \tilde{u}, \partial_{t} \tilde{\sigma}+\left[c \gamma \partial_{y}\left(\tilde{u}-a \tilde{\sigma}_{E}\right)\right]\right),
$$

and where the mappings $\left(F_{0}, H_{0}\right)$ are defined in (4.27). It follows from Theorem 3.4 and (4.31) that

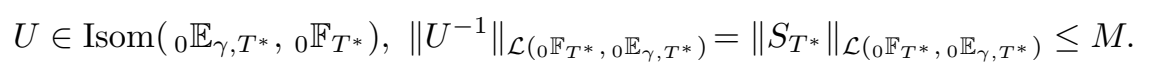

Next we obtain from (4.38), (4.40), and (4.43) that

$$
\left\|\left(D F_{0}\left(\bar{v}, \bar{\rho}_{E}\right), D H_{0}\left(\bar{v}, \bar{\rho}_{E}\right)\right)\right\|_{\left.\mathcal{L}_{(0} \mathbb{E}_{T^{*} \gamma},{ }_{0} \mathbb{F}_{T^{*}}\right)}<1 /(2 M) .
$$

Combining (5.17) and (5.18) yields

$$
D_{1} \Psi_{0}((\bar{v}, \bar{\rho}),(1,0)) \in \operatorname{Isom}\left({ }_{0} \mathbb{E}_{\gamma, T^{*},}{ }_{0} \mathbb{F}_{T^{*}}\right) .
$$

Again it is easily verified that $(u, \sigma) \in{ }_{0} \mathbb{B}_{\gamma, T^{*}}^{1}(0,2 r) \times{ }_{0} \mathbb{B}_{T^{*}}^{2}(0,2 r)$ is a solution to the system (5.14) if, and only if, the equation $\Psi_{0}((u, \sigma),(\lambda, \mu))=0$ holds true. 
Thus the implicit function theorem implies that there exists an open neighborhood of $(1,0)$ in $(1-\delta, 1+\delta) \times \mathbb{R}^{n}$, again denoted by $\Lambda$, such that

$$
\left[(\lambda, \mu) \mapsto\left(\bar{v}_{\lambda, \mu}, \bar{\rho}_{\lambda, \mu}\right)\right] \in C^{\omega}\left(\Lambda,{ }_{0} \mathbb{E}_{T^{*}}\right) .
$$

Observing that $\bar{w}_{\lambda, \mu}=\mathcal{T}\left(\bar{\rho}_{\lambda, \mu}, \lambda, \mu\right)$ we obtain from (5.10) that

$$
\left[(\lambda, \mu) \mapsto \bar{w}_{\lambda, \mu}\right] \in C^{\omega}\left(\Lambda,{ }_{0} \mathbb{E}_{T^{*}}^{1}\right) .
$$

(iv) Combining the results in step (iii) with Proposition 5.3 yields the assertion.

As an immediate consequence we get the following result.

Corollary 5.5. The function $\rho$ belongs to $C^{\omega}\left((0, T) \times \mathbb{R}^{n}\right)$.

Proof. Pick $\left(t_{0}, x_{0}\right) \in(0, T) \times \mathbb{R}^{n}$. From the embedding $\mathbb{E}_{T^{*}}^{2} \hookrightarrow C\left(I ; \operatorname{BUC}\left(\mathbb{R}^{n}\right)\right)$ and Theorem 5.4 we conclude that

$$
\left[(\lambda, \mu) \mapsto \rho_{\lambda, \mu}\right] \in C^{\omega}\left(\Lambda, C\left(I ; \operatorname{BUC}\left(\mathbb{R}^{n}\right)\right) .\right.
$$

Thus

$$
\left[(\lambda, \mu) \mapsto \rho\left(\lambda t_{0}, x_{0}+t_{0} \mu\right)\right] \in C^{\omega}(\Lambda, \mathbb{R})
$$

and this implies the assertion.

Proof of Theorem 1.1: Theorem 4.3 guarantees the existence of a unique solution $(v, \rho) \in \mathbb{E}_{T}$ of (2.5)-(2.6) and Corollary 5.5 implies that

$$
M=\bigcup_{t \in(0, T)}(\{t\} \times \Gamma(t)) \quad \text { is a real analytic manifold. }
$$

Moreover, Corollary 5.5 and well-known regularity results for linear parabolic equations show that the solution $\rho_{E}$ of $(2.6)$ is analytic, that is,

$$
\rho_{E}^{ \pm} \in C^{\omega}\left((0, T) \times \overline{\mathbb{R}_{ \pm}^{n+1}}\right) .
$$

This information, in turn, can now be used to conclude that $v$ is analytic as well. Indeed, we observe that $v$ solves the linear parabolic equation

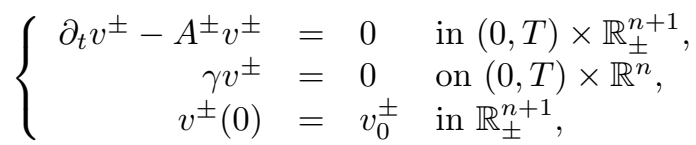

where

$$
\begin{aligned}
& A^{ \pm}=c_{ \pm} \Delta-c_{ \pm}\left(\frac{1+\left|\nabla_{x} \rho_{E}^{ \pm}\right|^{2}}{\left(1+\partial_{y} \rho_{E}^{ \pm}\right)^{2}}-1\right) \partial_{y}^{2}+c_{ \pm} \frac{2\left\langle\nabla_{x} \rho_{E}^{ \pm} \mid \nabla_{x} \partial_{y} \cdot\right\rangle}{1+\partial_{y} \rho_{E}^{ \pm}} \\
& +\frac{c_{ \pm}}{1+\partial_{y} \rho_{E}^{ \pm}}\left\{\left(\frac{1+\left|\nabla_{x} \rho_{E}^{ \pm}\right|^{2}}{\left(1+\partial_{y} \rho_{E}^{ \pm}\right)^{2}}-1\right) \partial_{y}^{2} \rho_{E}^{ \pm}-\frac{2\left\langle\nabla_{x} \rho_{E}^{ \pm} \mid \nabla_{x} \partial_{y} \rho_{E}^{ \pm}\right\rangle}{1+\partial_{y} \rho_{E}^{ \pm}}\right\} \partial_{y}
\end{aligned}
$$

is an elliptic differential operator with analytic coefficients. Hence we conclude once more from regularity theory for linear parabolic equations that

$$
v^{ \pm} \in C^{\omega}\left((0, T) \times \overline{\mathbb{R}_{ \pm}^{n+1}}\right) .
$$


It follows from (2.1)-(2.2) and (5.20) that

$$
\left.\Theta\right|_{(0, T) \times \overline{\mathbb{R}_{ \pm}^{n+1}}} \in \operatorname{Diff}^{\omega}\left((0, T) \times \overline{\mathbb{R}_{ \pm}^{n+1}}, \bar{\Omega}_{T}^{ \pm}\right) .
$$

Let $u:=\Theta_{*} v$. The assertions of Theorem 1.1 are now a consequence of Theorem 4.3 and of $(5.19)-(5.21)$.

Remark 5.6. The idea to introduce parameters and use the implicit function theorem to obtain regularity results for solutions of parabolic equations was first conceived by Masuda [42], and later rediscovered independently by Angenent [2]. Translations were first introduced in Escher-Simonett [24] to obtain spacial regularity for solutions to a free boundary problem. This approach has been generalized in various directions $[25,26]$.

\section{Appendix}

Here we establish some basic and important facts about extension operators and embeddings that we used in our calculations in the previous sections. We start with a result on the existence of an extension operator $\mathcal{E}:{ }_{0} W_{p}^{r}(J ; X) \rightarrow{ }_{0} W_{p}^{r}\left(\mathbb{R}_{+} ; X\right)$. The cruical point here is the independence of the norm of the operator of the length of the interval $J$.

Proposition 6.1. Let $X$ be a Banach space. Suppose $T_{0}>0$ is a fixed number and $r \in(1 / p, 1]$. Then there exists an extension operator

$$
\mathcal{E}_{J, r}:{ }_{0} W_{p}^{r}(J ; X) \rightarrow{ }_{0} W_{p}^{r}\left(\mathbb{R}_{+} ; X\right)
$$

and a constant $c_{0}=c_{0}\left(r, p, T_{0}\right)$ such that

$$
\left\|\mathcal{E}_{J, r} u\right\|_{0 W_{p}^{r}\left(\mathbb{R}_{+} ; X\right)} \leq c_{0}\|u\|_{0 W_{p}^{r}(J ; X)}
$$

for every $u \in{ }_{0} W_{p}^{r}(J ; X)$ and $T \leq T_{0}$, where $J=[0, T]$. The family $\left\{\mathcal{E}_{J, r}\right\}_{r \in(1 / p, 1]}$ is compatible, i.e. for $r, s \in(1 / p, 1]$ we have

$$
\mathcal{E}_{J, r} u=\mathcal{E}_{J, s} u, \quad u \in{ }_{0} W_{p}^{r}(J ; X) \cap{ }_{0} W_{p}^{s}(J ; X) .
$$

Proof. Let $T \in\left(0, T_{0}\right)$ and $u: J \rightarrow X$ be given. We set

$$
\mathcal{E} u(t):=\mathcal{E}_{J, r} u(t):= \begin{cases}u(t) & \text { if } 0 \leq t \leq T, \\ u(2 T-t) & \text { if } T \leq t \leq 2 T, \\ 0 & \text { if } 2 T \leq t .\end{cases}
$$

It is clear that $\|\mathcal{E} u\|_{L_{p}\left(\mathbb{R}_{+} ; X\right)} \leq 2\|\mathcal{E} u\|_{L_{p}(J ; X)}$ for $u \in L_{p}(J ; X)$. Let $u \in{ }_{0} W_{p}^{r}(J ; X)$ be given. In order to prove the assertion for $r \in(1 / p, 1)$ we only need to show that

$$
\begin{aligned}
\langle\mathcal{E} u\rangle_{I_{1} \times I_{2}, r, p}: & =\int_{I_{2}} \int_{I_{1}} \frac{\|\mathcal{E} u(t)-\mathcal{E} u(s)\|_{X}^{p}}{|t-s|^{1+r p}} \mathrm{~d} s \mathrm{~d} t \\
& \leq c_{0} \int_{0}^{T} \int_{0}^{T} \frac{\|u(t)-u(s)\|_{X}^{p}}{|t-s|^{1+r p}} \mathrm{~d} s \mathrm{~d} t
\end{aligned}
$$

for $I_{1}=I_{2}=\mathbb{R}_{+}$, see (3.1). It is clear that

$$
\langle\mathcal{E} u\rangle_{I \times I, r, p}=\langle u\rangle_{J \times J, r, p} \quad \text { for } \quad I=J \quad \text { and } \quad I=(T, 2 T) .
$$


An easy computation shows that

$$
\begin{aligned}
\langle\mathcal{E} u\rangle_{(0, T) \times(T, 2 T), r, p} & =\int_{0}^{T} \int_{0}^{T} \frac{\|u(t)-u(s)\|_{X}^{p}}{|t+s-2 T|^{1+r p}} \mathrm{~d} s \mathrm{~d} t \\
& \leq \int_{0}^{T} \int_{0}^{T} \frac{\|u(t)-u(s)\|_{X}^{p}}{|t-s|^{1+r p}} \mathrm{~d} s \mathrm{~d} t .
\end{aligned}
$$

Next we have

$$
\langle\mathcal{E} u\rangle_{(0, T) \times(2 T, \infty), r, p}=\int_{0}^{T} \int_{2 T}^{\infty} \frac{\|u(t)\|_{X}^{p}}{|t-s|^{1+r p}} \mathrm{~d} s \mathrm{~d} t \leq \frac{1}{r p} \int_{0}^{T} \frac{\|u(t)\|_{X}^{p}}{t^{r p}} \mathrm{~d} t .
$$

Moreover

$$
\begin{aligned}
\langle\mathcal{E} u\rangle_{(T, 2 T) \times(2 T, \infty), r, p} & =\int_{0}^{T} \int_{2 T}^{\infty} \frac{\|u(t)\|_{X}^{p}}{|2 T-t-s|^{1+r p}} \mathrm{~d} s \mathrm{~d} t \\
& =\int_{0}^{T} \int_{0}^{\infty} \frac{\|u(t)\|_{X}^{p}}{(t+s)^{1+r p}} \mathrm{~d} s \mathrm{~d} t=\frac{1}{r p} \int_{0}^{T} \frac{\|u(t)\|_{X}^{p}}{t^{r p}} \mathrm{~d} t .
\end{aligned}
$$

We now suppose that $u$ is smooth with compact support contained in $(0, T]$. Then

$$
\begin{aligned}
& \left(\int_{0}^{T} \frac{1}{t^{r p}}\|u(t)\|_{X}^{p} \mathrm{~d} t\right)^{1 / p} \leq\left(\int_{0}^{T}\left[\int_{0}^{t}\|u(t)-u(s)\|_{X} \mathrm{~d} s+\int_{0}^{t}\|u(s)\|_{X} \mathrm{~d} s\right]^{p} \frac{\mathrm{d} t}{t^{(1+r) p}}\right)^{1 / p} \\
& \leq\left(\int_{0}^{T} \frac{t^{p / p^{\prime}}}{t^{(1+r) p}} \int_{0}^{t}\|u(t)-u(s)\|_{X}^{p} \mathrm{~d} s \mathrm{~d} t\right)^{1 / p}+\left(\int_{0}^{T} \frac{1}{t^{(1+r) p}}\left[\int_{0}^{t}\|u(s)\|_{X} \mathrm{~d} s\right]^{p} \mathrm{~d} t\right)^{1 / p} \\
& \leq\left(\int_{0}^{T} \int_{0}^{T} \frac{\|u(t)-u(s)\|_{X}^{p}}{|t-s|^{1+r p}} \mathrm{~d} s \mathrm{~d} t\right)^{1 / p}+\left(\int_{0}^{T} \frac{1}{t^{(1+r) p}} \int_{0}^{t}\|u(s)\|_{X}^{p} \mathrm{~d} s \mathrm{~d} t\right)^{1 / p}
\end{aligned}
$$

where we first used Minkowski's inequality on $(0, T)$ with respect to the measure $\mathrm{d} t / t^{(1+r) p}$ and then Hölder's inequality on $(0, t)$ with respect to $\mathrm{d} s$ in the second line. For the last term we may use Hardy's inequality to the result

$$
\left(\int_{0}^{T} \frac{1}{t^{(1+r) p}}\left[\int_{0}^{t}\|u(s)\|_{X} \mathrm{~d} s\right]^{p} \mathrm{~d} t\right)^{1 / p} \leq \frac{1}{(1+r-1 / p)}\left(\int_{0}^{T} \frac{1}{t^{r p}}\|u(t)\|_{X}^{p} \mathrm{~d} t\right)^{1 / p}
$$

and combining the last estimates yields

$$
\left(\int_{0}^{T} \frac{1}{t^{r p}}\|u(t)\|_{X}^{p} \mathrm{~d} t\right)^{1 / p} \leq \frac{(1+r-1 / p)}{(r-1 / p)}\left(\int_{0}^{T} \int_{0}^{T} \frac{\|u(t)-u(s)\|_{X}^{p}}{|t-s|^{1+r p}} \mathrm{~d} s \mathrm{~d} t\right)^{1 / p}
$$

provided that $r>1 / p$. By an approximation argument inequality (6.8) remains true for every function $u \in{ }_{0} W_{p}^{r}([0, T], X)$ with $r>1 / p$.

By symmetry it is now clear that the estimates (6.4)-(6.8) yield (6.3). The case $r=1$ is not difficult to verify, and this completes the proof. 
Proposition 6.1 is an essential ingredient in order to prove the independence of the embedding constants on $J$ in the following embeddings in case we assume vanishing time trace at $t=0$.

Proposition 6.2. Suppose $T_{0} \in(0, \infty]$ is a fixed number, and $I:=\left[0, T_{0}\right]$.

(a) Let $r \in(1 / p, 1]$ and let $X$ be a Banach space. Then

$$
W_{p}^{r}(I ; X) \hookrightarrow \operatorname{BUC}(I ; X)
$$

and there is a constant $c_{0}=c\left(T_{0}\right)$ such that

$$
\|u\|_{o \mathrm{BUC}(J ; X)} \leq c_{0}\|u\|_{\mathrm{o}} W_{p}^{r}(J ; X)
$$

for every $u \in{ }_{0} W_{p}^{r}(J ; X)$ and $T \leq T_{0}$, where $J=[0, T]$.

(b) Let $\Omega \subset \mathbb{R}^{m}$ be a smooth domain. Then

$$
W_{p}^{1}\left(I ; L_{p}(\Omega) \cap L_{p}\left(I ; W_{p}^{2}(\Omega)\right) \hookrightarrow \operatorname{BUC}\left(I ; W_{p}^{2-2 / p}(\Omega)\right)\right.
$$

and there is a constant $c_{0}=c_{0}\left(T_{0}\right)$ such that

$$
\|u\|_{0} B U C\left(J ; W_{p}^{2-2 / p}(\Omega)\right) \leq c_{0}\|u\|_{0 W_{p}^{1}\left(J ; L_{p}(\Omega) \cap L_{p}\left(J ; W_{p}^{2}(\Omega)\right)\right.}
$$

for every $u \in{ }_{0} W_{p}^{1}\left(J ; L_{p}(\Omega)\right) \cap L_{p}\left(J ; W_{p}^{2}(\Omega)\right)$ and $T \leq T_{0}$, where $J=[0, T]$.

(c) Let $p>n+3$. Then

$$
W_{p}^{1 / 2-1 / 2 p}\left(I ; L_{p}\left(\mathbb{R}^{n}\right)\right) \cap L_{p}\left(I ; W_{p}^{1-1 / p}\left(\mathbb{R}^{n}\right)\right) \hookrightarrow \operatorname{BUC}\left(I \times \mathbb{R}^{n}\right)
$$

and there exists a constant $c_{0}=c_{0}\left(T_{0}\right)$ such that

$$
\|g\|_{\mathrm{BUC}\left(J \times \mathbb{R}^{n}\right)} \leq c_{0}\|g\|_{{ }_{0} W_{p}^{1 / 2-1 / 2 p}\left(J ; L_{p}\left(\mathbb{R}^{n}\right)\right) \cap L_{p}\left(J ; W_{p}^{1-1 / p}\left(\mathbb{R}^{n}\right)\right)}
$$

for every $g \in{ }_{0} W_{p}^{1 / 2-1 / 2 p}\left(J ; L_{p}\left(\mathbb{R}^{n}\right)\right) \cap L_{p}\left(J ; W_{p}^{1-1 / p}\left(\mathbb{R}^{n}\right)\right)$ and $T \leq T_{0}$, where $J=[0, T]$.

Proof. (a) The first assertion is a consequence of Sobolev's embedding theorem, and the uniform estimate in (6.9) is obtained by the following commutative diagram

$$
\begin{array}{ccc}
{ }_{0} W_{p}^{r}(J ; X) & \stackrel{\mathcal{E}_{J}}{\longrightarrow} & { }_{0} W_{p}^{r}\left(\mathbb{R}_{+} ; X\right) \\
\downarrow & & \downarrow \\
{ }_{0} B U C(J ; X) & \stackrel{\mathcal{R}_{J}}{\longleftarrow} & { }_{0} B U C\left(\mathbb{R}_{+} ; X\right)
\end{array}
$$

where $\mathcal{E}_{J}$ is the extension operator of Proposition 6.1 , and $\mathcal{R}_{J}$ denotes the restriction operator.

(b) The first assertion can, for instance, be found in [1, Theroem III.4.10.2], and the uniform estimate in (6.10) can be proved along the same lines as in (a), with ${ }_{0} W_{p}^{r}(I ; X)$ replaced by ${ }_{0} W_{p}^{1}\left(I ; L_{p}(\Omega) \cap L_{p}\left(I ; W_{p}^{2}(\Omega)\right)\right.$ for $I=J, \mathbb{R}_{+}$.

(c) The first assertion is contained in [27, Remark 5.3(d)], and the uniform estimate in (6.11) can, once more, be obtained by an analogous argument as in (a).

Lemma 6.3. Let $J=[0, T]$. Then we have the following embedding

$$
W_{p}^{1}\left(J ; W_{p}^{1-1 / p}\left(\mathbb{R}^{n}\right)\right) \cap L_{p}\left(J ; W_{p}^{2-1 / p}\left(\mathbb{R}^{n}\right)\right) \hookrightarrow W_{p}^{1 / 2-1 / 2 p}\left(J ; W_{p}^{1}\left(\mathbb{R}^{n}\right)\right) .
$$


Proof. (i) We will first assume that $J=\mathbb{R}$. Let $\mathcal{K} \in\{H, W\}$ and let $r \in \mathbb{R}$. Then we define the operators $A, B$ in $X:=L_{p}\left(\mathbb{R} ; \mathcal{K}_{p}^{r}\left(\mathbb{R}^{n}\right)\right)$ by

$$
\begin{array}{ll}
A u:=(1-\Delta)^{1 / 2} u, & u \in \mathrm{D}(A)=L_{p}\left(\mathbb{R} ; \mathcal{K}_{p}^{r+1}\left(\mathbb{R}^{n}\right)\right) \\
B u:=\left(\partial_{t}+1\right) u, & u \in \mathrm{D}(B)=W_{p}^{1}\left(\mathbb{R} ; \mathcal{K}_{p}^{r}\left(\mathbb{R}^{n}\right)\right) .
\end{array}
$$

It is well-known that $A, B \in B I P(X)$ with power angles $\theta_{A}=0, \theta_{B}=\pi / 2$. Thus, by the Dore-Venni theorem, see for instance [47, Theorem 8.4],

$$
A+B: W_{p}^{1}\left(\mathbb{R} ; \mathcal{K}_{p}^{r}\left(\mathbb{R}^{n}\right)\right) \cap L_{p}\left(\mathbb{R} ; \mathcal{K}_{p}^{r+1}\left(\mathbb{R}^{n}\right)\right) \rightarrow L_{p}\left(\mathbb{R} ; \mathcal{K}_{p}^{r}\left(\mathbb{R}^{n}\right)\right)
$$

is an isomorphism. Choosing $\mathcal{K}=H$ we also have

$$
\begin{aligned}
& \mathrm{D}\left(A^{\alpha}\right)=[X, D(A)]_{\alpha}=L_{p}\left(\mathbb{R} ;\left[H_{p}^{r}\left(\mathbb{R}^{n}\right), H_{p}^{r+1}\left(\mathbb{R}^{n}\right)\right]_{\alpha}\right)=L_{p}\left(\mathbb{R} ; H_{p}^{r+\alpha}\left(\mathbb{R}^{n}\right)\right), \\
& \mathrm{D}\left(B^{\alpha}\right)=[X, D(B)]_{\alpha}=H_{p}^{\alpha}\left(\mathbb{R} ; H_{p}^{r}\left(\mathbb{R}^{n}\right)\right)
\end{aligned}
$$

see [47, Theorem 8.1]. The mixed derivative theorem [27, Lemma 9.7], and the invertibility of $A: \mathrm{D}(A) \rightarrow X$ and $B: \mathrm{D}(B) \rightarrow X$ now yield

$$
\begin{aligned}
\|u\|_{H_{p}^{s}\left(\mathbb{R} ; H_{p}^{r+(1-s)}\left(\mathbb{R}^{n}\right)\right)} & \leq C\left\|B^{s} u\right\|_{L_{p}\left(\mathbb{R} ; H_{p}^{r+(1-s)}\left(\mathbb{R}^{n}\right)\right)} \leq C\left\|A^{1-s} B^{s} u\right\|_{X} \\
& \leq C\|(A+B) u\|_{X} \leq C\|u\|_{\mathrm{D}(A) \cap \mathrm{D}(B)} .
\end{aligned}
$$

In particular, we have

$$
\begin{aligned}
& E_{0}:=H_{p}^{1}\left(\mathbb{R}_{+} ; H_{p}^{r_{0}}\left(\mathbb{R}^{n}\right)\right) \cap L_{p}\left(\mathbb{R}_{+} ; H_{p}^{r_{0}+1}\left(\mathbb{R}^{n}\right)\right) \hookrightarrow H_{p}^{r_{0}}\left(\mathbb{R}_{+} ; H_{p}^{1}\left(\mathbb{R}^{n}\right)\right), \\
& E_{1}:=H_{p}^{1}\left(\mathbb{R}_{+} ; H_{p}^{r_{1}}\left(\mathbb{R}^{n}\right)\right) \cap L_{p}\left(\mathbb{R}_{+} ; H_{p}^{r_{1}+1}\left(\mathbb{R}^{n}\right)\right) \hookrightarrow H_{p}^{r_{1}}\left(\mathbb{R}_{+} ; H_{p}^{1}\left(\mathbb{R}^{n}\right)\right) .
\end{aligned}
$$

By interpolating these embeddings by the real method $(\cdot, \cdot)_{1 / 2, p}$ with $r_{0}=r-\varepsilon$ and $r_{1}=r+\varepsilon$ we obtain

$$
\left(E_{0}, E_{1}\right)_{1 / 2, p} \hookrightarrow W_{p}^{r}\left(\mathbb{R} ; H_{p}^{1}\left(\mathbb{R}^{n}\right)\right) .
$$

We can now infer from (6.14) and interpolation theory that

$$
\begin{aligned}
& A+B:\left(E_{0}, E_{1}\right)_{1 / 2, p} \rightarrow L_{p}\left(\mathbb{R} ; W_{p}^{r}\left(\mathbb{R}^{n}\right)\right) \\
& A+B: W_{p}^{1}\left(\mathbb{R} ; W_{p}^{r}\left(\mathbb{R}^{n}\right)\right) \cap L_{p}\left(\mathbb{R} ; W_{p}^{r+1}\left(\mathbb{R}^{n}\right)\right) \rightarrow L_{p}\left(\mathbb{R} ; W_{p}^{r}\left(\mathbb{R}^{n}\right)\right)
\end{aligned}
$$

are isomorphisms. Consequently,

$$
I:=(A+B)^{-1}(A+B):\left(E_{0}, E_{1}\right)_{1 / 2, p} \rightarrow W_{p}^{1}\left(\mathbb{R} ; W_{p}^{r}\left(\mathbb{R}^{n}\right)\right) \cap L_{p}\left(\mathbb{R} ; W_{p}^{r+1}\left(\mathbb{R}^{n}\right)\right)
$$

is an isomorphim as well, and we conclude from (6.15) that

$$
W_{p}^{1}\left(\mathbb{R} ; W_{p}^{r}\left(\mathbb{R}^{n}\right)\right) \cap L_{p}\left(\mathbb{R} ; W_{p}^{r+1}\left(\mathbb{R}^{n}\right)\right) \hookrightarrow W_{p}^{r}\left(\mathbb{R} ; W_{p}^{1}\left(\mathbb{R}^{n}\right)\right) .
$$

Choosing $r=1-1 / p$ and using that

$$
W_{p}^{1-1 / p}\left(\mathbb{R} ; W_{p}^{1}\left(\mathbb{R}^{n}\right)\right) \hookrightarrow W_{p}^{1 / 2-1 / 2 p}\left(\mathbb{R} ; W_{p}^{1}\left(\mathbb{R}^{n}\right)\right)
$$

we obtain (6.13) for $J=\mathbb{R}$.

(ii) Suppose now that $J=[0, T]$ and let

$$
R^{c} \in \mathcal{L}\left(\mathcal{F}_{p}^{s}(J ; X), \mathcal{F}_{p}^{s}(\mathbb{R} ; X)\right)
$$

be an appropriate extension operator, where $\mathcal{F} \in\{H, W\}, X$ is a Banach space and, say, $0 \leq s \leq 1$. Then the result for $J=[0, T]$ follows by first extending the functions to $I=\mathbb{R}$, using (6.16), and then restricting again to $J$. 
The reduction of problem (3.2)-(3.3) to the case of vanishing times traces at $t=0$ in the proof of Theorem 3.4 was based on the following result.

Lemma 6.4. Let $1<p<\infty, T \in(0, \infty]$, and $J=[0, T]$.

(i) For each $\sigma_{0} \in W_{p}^{2-2 / p}\left(\mathbb{R}^{n}\right)$ there exists an extension

$$
\eta_{1} \in W_{p}^{3 / 2-1 / 2 p}\left(J ; L_{p}\left(\mathbb{R}^{n}\right)\right) \cap W_{p}^{1}\left(J ; W_{p}^{1-1 / p}\left(\mathbb{R}^{n}\right)\right) \cap L_{p}\left(J ; W_{p}^{2-1 / p}\left(\mathbb{R}^{n}\right)\right)
$$
such that $\eta_{1}(0)=\sigma_{0}$ and, if $p>3$, also that $\partial_{t} \eta_{1}(0)=0$.

(ii) Suppose $p>3$. Then for each $\sigma_{0} \in W_{p}^{2-2 / p}\left(\mathbb{R}^{n}\right)$ and $\sigma_{1} \in W_{p}^{1-3 / p}\left(\mathbb{R}^{n}\right)$ there exists an extension $\eta_{2}$ with the same regularity properties as $\eta_{1}$ satisfying $\eta_{2}(0)=\sigma_{0}$ and $\partial_{t} \eta_{2}(0)=\sigma_{1}$.

Proof. (i) Let $1<p<\infty$. We claim that

$$
\eta_{1}(t):=\left(2 \mathrm{e}^{-t\left(1-\Delta_{x}\right)^{\frac{1}{2}}}-\mathrm{e}^{-2 t\left(1-\Delta_{x}\right)^{\frac{1}{2}}}\right) \sigma_{0}
$$

satisfies the properties asserted in (i). We have

$$
\mathrm{e}^{-k t\left(1-\Delta_{x}\right)^{\frac{1}{2}}} \sigma_{0} \in W_{p}^{1}\left(J ; W_{p}^{1-1 / p}\left(\mathbb{R}^{n}\right)\right) \cap L_{p}\left(J ; W_{p}^{2-1 / p}\left(\mathbb{R}^{n}\right)\right)
$$

for $k=1,2$. By Proposition 6.3 we know that the latter space is continuously embedded in $W_{p}^{1 / 2-1 / 2 p}\left(J ; W_{p}^{1}\left(\mathbb{R}^{n}\right)\right)$. This implies that

$$
\partial_{t} \mathrm{e}^{-k t\left(1-\Delta_{x}\right)^{\frac{1}{2}}} \sigma_{0}=-k\left(1-\Delta_{x}\right)^{\frac{1}{2}} \mathrm{e}^{-k t\left(1-\Delta_{x}\right)^{\frac{1}{2}}} \sigma_{0} \in W_{p}^{1 / 2-1 / 2 p}\left(J ; L_{p}\left(\mathbb{R}^{n}\right)\right) .
$$

Consequently,

$$
\eta_{1} \in W_{p}^{3 / 2-1 / 2 p}\left(J ; L_{p}\left(\mathbb{R}^{n}\right)\right) \cap W_{p}^{1}\left(J ; W_{p}^{1-1 / p}\left(\mathbb{R}^{n}\right)\right) \cap L_{p}\left(J ; W_{p}^{2-1 / p}\left(\mathbb{R}^{n}\right)\right) .
$$

Obviously $\eta_{1}(0)=\sigma_{0}$. If $p>3$, the time trace at $t=0$ of $\partial_{t} \eta_{1}$ is well defined and we also have $\partial_{t} \eta_{1}(0)=0$. This proves $(\mathrm{i})$.

(ii) Now suppose $p>3$. We set

$$
\eta_{2}(t):=\eta_{1}(t)+\left(\mathrm{e}^{-t\left(1-\Delta_{x}\right)}-\mathrm{e}^{-2 t\left(1-\Delta_{x}\right)}\right)\left(1-\Delta_{x}\right)^{-1} \sigma_{1} .
$$

Since $\left(1-\Delta_{x}\right)^{-1} \sigma_{1} \in W_{p}^{3-3 / p}\left(\mathbb{R}^{n}\right)$, we obtain

$$
\mathrm{e}^{-k t\left(1-\Delta_{x}\right)}\left(1-\Delta_{x}\right)^{-1} \sigma_{1} \in W_{p}^{1}\left(J ; W_{p}^{1-1 / p}\left(\mathbb{R}^{n}\right)\right) \cap L_{p}\left(J ; W_{p}^{3-1 / p}\left(\mathbb{R}^{n}\right)\right)
$$

for $k=1,2$. Furthermore,

$$
\partial_{t} \eta_{2}(t)=\partial_{t} \eta_{1}(t)+\left(2 \mathrm{e}^{-2 t\left(1-\Delta_{x}\right)}-\mathrm{e}^{-t\left(1-\Delta_{x}\right)}\right) \sigma_{1} .
$$

It follows from

$$
\mathrm{e}^{-k t\left(1-\Delta_{x}\right)} \sigma_{1} \in W_{p}^{1 / 2-1 / 2 p}\left(J ; L_{p}\left(\mathbb{R}^{n}\right)\right) \cap L_{p}\left(J ; W_{p}^{1-1 / p}\left(\mathbb{R}^{n}\right)\right),
$$

see [27, page 23], and from the regularity for $\eta_{1}$ that

$$
\partial_{t} \eta_{2} \in W_{p}^{1 / 2-1 / 2 p}\left(J ; L_{p}\left(\mathbb{R}^{n}\right)\right) \cap L_{p}\left(J ; W_{p}^{1-1 / p}\left(\mathbb{R}^{n}\right)\right) .
$$

Moreover, $\eta_{2}(0)=\sigma_{0}$ and $\partial_{t} \eta_{2}(0)=\sigma_{1}$, which proves (ii).

Remark 6.5. We would like to mention that the extension $\eta$ was motivated by [53, Lemma 1].

Finally, we collect some basic facts about the space $\mathbb{F}_{T}^{2}$, introduced in (4.2) and frequently used in Section 4. 
Lemma 6.6. Let $p>n+3$. Then

(i)

$$
\mathbb{F}_{T}^{2} \hookrightarrow \mathrm{BUC}\left(J \times \mathbb{R}^{n}\right)
$$

In particular, if we replace $\mathbb{F}_{T}^{2}$ by ${ }_{0} \mathbb{F}_{T}^{2}$, then there is a constant $c_{0}=c_{0}\left(T_{0}\right)$ such that

$$
\|g\|_{\mathrm{BUC}\left(J \times \mathbb{R}^{n}\right)} \leq c_{0}\|g\|_{{ }_{0} \mathbb{F}_{T}^{2}}, \quad g \in{ }_{0} \mathbb{F}_{T}^{2},
$$

for all $T \leq T_{0}$.

(ii) The space $\mathbb{F}_{T}^{2}$ is a multiplication algebra.

(iii) The set

$$
\mathbb{F}_{T, b}^{2}:=\left\{g \in \mathbb{F}_{T}^{2}:\|g\|_{L_{\infty}\left(J \times \mathbb{R}^{n}\right)}<b\right\}, \quad b>0,
$$

is open in $\mathbb{F}_{T}^{2}$ and

$$
\|g h\|_{\mathbb{F}_{T}^{2}} \leq \max \{a, b\}\left(\|g\|_{\mathbb{F}_{T}^{2}}+\|h\|_{\mathbb{F}_{T}^{2}}\right), \quad(g, h) \in \mathbb{F}_{T, a}^{2} \times \mathbb{F}_{T, b}^{2} .
$$

(iv) There exists a constant $c_{0}=c_{0}\left(T_{0}\right)$ such that

$$
\|g h\|_{\mathbb{F}_{T}^{2}} \leq c_{0}\left(\|g\|_{\infty}+\|g\|_{\mathbb{F}_{T}^{2}}\right)\|h\|_{\mathbb{F}_{T}^{2}}, \quad(g, h) \in \mathbb{F}_{T}^{2} \times{ }_{0} \mathbb{F}_{T}^{2},
$$

for all $T \leq T_{0}$.

(v) We have

$$
\left\|\frac{g}{1+h}\right\|_{\mathbb{F}_{T}^{2}} \leq 2\left(\|g\|_{\mathbb{F}_{T}^{2}}+2 a\|h\|_{\mathbb{F}_{T}^{2}}\right), \quad(g, h) \in \mathbb{F}_{T, a}^{2} \times \mathbb{F}_{T, b}^{2}
$$

for any $b \leq 1 / 2$.

(vi) $[(g, h) \mapsto g /(1+h)] \in C^{\omega}\left(\mathbb{F}_{T}^{2} \times \mathbb{F}_{T, 1 / 2}^{2}, \mathbb{F}_{T}^{2}\right)$.

Proof. (i) The assertion follows from Proposition 6.2(c).

(ii) Recall that the norm in $W_{p}^{r}\left(J ; L_{p}\left(\mathbb{R}^{n}\right)\right)$ for $r \in(0,1)$ is given by

$$
\|g\|_{r, p, L_{p}}:=\|g\|_{W_{p}^{r}\left(J ; L_{p}\left(\mathbb{R}^{n}\right)\right)}=\|g\|_{p}+\langle g\rangle_{r, p, L_{p}},
$$

where $\|g\|_{p}=\|g\|_{L_{p}\left(J ; L_{p}\left(\mathbb{R}^{n}\right)\right)}$ and

$$
\langle g\rangle_{r, p, L_{p}}=\left(\int_{J} \int_{J} \frac{\|g(t)-g(s)\|_{L_{p}\left(\mathbb{R}^{n}\right)}^{p}}{|t-s|^{1+r p}} \mathrm{~d} \mathrm{~d} s\right)^{1 / p} .
$$

By writing

$$
g(t) h(t)-g(s) h(s)=(g(t)-g(s)) h(t)+g(s)(h(t)-h(s)),
$$

we deduce for $r=1 / 2-1 / 2 p$ in view of (i)

$$
\begin{aligned}
\|g h\|_{r, p, L_{p}} & \leq\|g\|_{\infty}\|h\|_{p}+\|h\|_{\infty}\langle g\rangle_{r, p, L_{p}}+\|g\|_{\infty}\langle h\rangle_{r, p, L_{p}} \\
& \leq C_{T}\|g\|_{\mathbb{F}_{T}^{2}}\|h\|_{\mathbb{F}_{T}^{2}}, \quad g, h \in \mathbb{F}_{T}^{2} .
\end{aligned}
$$

As the norm of $L_{p}\left(J ; W_{p}^{s}\left(\mathbb{R}^{n}\right)\right)$, the second space of $\mathbb{F}_{T}^{2}$ for $s=1-1 / p$, is given by

$$
\|g\|_{L_{p}\left(J ; W_{p}^{s}\left(\mathbb{R}^{n}\right)\right)}=\left(\int_{J}\left(\|g(t)\|_{L_{p}\left(\mathbb{R}^{n}\right)}^{p}+\langle g(t)\rangle_{s, p}^{p}\right) \mathrm{d} t\right)^{1 / p},
$$


where

$$
\langle g(t)\rangle_{s, p}=\left(\int_{\mathbb{R}^{n}} \int_{\mathbb{R}^{n}} \frac{\left|g(t, x)-g\left(t, x^{\prime}\right)\right|^{p}}{\left|x-x^{\prime}\right|^{n+s p}} \mathrm{~d} x \mathrm{~d} x^{\prime}\right)^{1 / p},
$$

an analogous calculation as above gives

$$
\|g(t) h(t)\|_{\left.W_{p}^{s}\left(\mathbb{R}^{n}\right)\right)} \leq\|g\|_{\infty}\|h(t)\|_{p}+\|h\|_{\infty}\langle g(t)\rangle_{s, p}+\|g\|_{\infty}\langle h(t)\rangle_{s, p},
$$

which implies

$$
\|g h\|_{L_{p}\left(J ; W_{p}^{s}\left(\mathbb{R}^{n}\right)\right)} \leq C_{T}\|g\|_{\mathbb{F}_{T}^{2}}\|h\|_{\mathbb{F}_{T}^{2}}, \quad g, h \in \mathbb{F}_{T}^{2} .
$$

Combining (6.20) and (6.22) we arrive at (ii).

(iii) It is easy to see that the set $\mathbb{F}_{T, b}^{2}$ is open in $\mathbb{F}_{T}^{2}$, and the estimate in (iii) follows from the first line in (6.20) and from (6.21).

(iv) By (i) we immediately get

$$
\|h\|_{\mathrm{BUC}\left(J \times \mathbb{R}^{n}\right)} \leq C\left(T_{0}\right)\|h\|_{{ }_{0} \mathbb{F}_{T}^{2}}, \quad h \in{ }_{0} \mathbb{F}_{T}^{2}, \quad T \leq T_{0} .
$$

But then (iv) as well is a consequence of the first line in (6.20) and of (6.21).

(v) In order to see (v) note that $h \in \mathbb{F}_{T, b}^{2}$ with $b \leq 1 / 2$ implies

$$
\left\|\frac{1}{1+h}\right\|_{\infty} \leq 2
$$

Hence for $r=1 / 2-1 / 2 p$ we may compute

$$
\begin{aligned}
\left\|\frac{g}{1+h}\right\|_{r, p, L_{p}} & \leq\left\|\frac{1}{1+h}\right\|_{\infty}\|g\|_{p}+\left\|\frac{1}{1+h}\right\|_{\infty}\langle g\rangle_{r, p, L_{p}}+\|g\|_{\infty}\left\langle\frac{1}{1+h}\right\rangle_{r, p, L_{p}} \\
& \leq 2\|g\|_{r, p, L_{p}}+a\left\langle\frac{1}{1+h}\right\rangle_{r, p, L_{p}} .
\end{aligned}
$$

Moreover,

$$
\begin{aligned}
\left\langle\frac{1}{1+h}\right\rangle_{r, p, L_{p}} & =\left(\int_{J} \int_{J}\left\|\frac{h(s)-h(t)}{(1+h(t))(1+h(s))}\right\|_{L_{p}\left(\mathbb{R}^{n}\right)}^{p} \frac{\mathrm{d} t \mathrm{~d} s}{|t-s|^{1+r p}}\right)^{1 / p} \\
& \leq\left\|\frac{1}{1+h}\right\|_{\infty}^{2}\langle h\rangle_{r, p, L_{p}} \leq 4\langle h\rangle_{r, p, L_{p}},
\end{aligned}
$$

which implies

$$
\left\|\frac{g}{1+h}\right\|_{r, p, L_{p}} \leq 2\left(\|g\|_{r, p, L_{p}}+2 a\|h\|_{r, p, L_{p}}\right), \quad(g, h) \in \mathbb{F}_{T, a}^{2} \times \mathbb{F}_{T, b}^{2} .
$$

Similarly we can obtain

$$
\begin{aligned}
\left\|\frac{g(t)}{1+h(t)}\right\|_{W_{p}^{s}\left(\mathbb{R}^{n}\right)} \leq & \left\|\frac{1}{1+h}\right\|_{\infty}\|g(t)\|_{L_{p}\left(\mathbb{R}^{n}\right)}+\left\|\frac{1}{1+h}\right\|_{\infty}\langle g(t)\rangle_{s, p} \\
& +\|g\|_{\infty}\left\langle\frac{1}{1+h(t)}\right\rangle_{s, p} \\
\leq & 2\left(\|g(t)\|_{W_{p}^{s}\left(\mathbb{R}^{n}\right)}+2 a\|h(t)\|_{W_{p}^{s}\left(\mathbb{R}^{n}\right)}\right) .
\end{aligned}
$$


Consequently,

$$
\left\|\frac{g}{1+h}\right\|_{L_{p}\left(J ; W_{p}^{s}\left(\mathbb{R}^{n}\right)\right)} \leq 2\left(\|g\|_{L_{p}\left(J ; W_{p}^{s}\left(\mathbb{R}^{n}\right)\right)}+2 a\|h\|_{L_{p}\left(J ; W_{p}^{s}\left(\mathbb{R}^{n}\right)\right)}\right)
$$

for all $(g, h) \in \mathbb{F}_{T, a}^{2} \times \mathbb{F}_{T, b}^{2}$. The relations (6.23) and (6.24) then yield (v).

(vi) A formal calculation, based on the geometric series, shows that

$$
\frac{g}{1+h}=\frac{g}{1+h_{0}}+\frac{g}{1+h_{0}} \sum_{k=1}^{\infty}(-1)^{k}\left(\frac{h-h_{0}}{1+h_{0}}\right)^{k}
$$

for $h_{0}, h \in \mathbb{F}_{T, 1 / 2}^{2}$. Given $k \in \mathbb{N}, k \geq 1$, we define

$$
P_{k}\left(h_{1}, \ldots, h_{k}\right):=\left(\frac{h_{1}}{1+h_{0}}\right) \cdots\left(\frac{h_{k}}{1+h_{0}}\right), \quad h_{1}, \ldots, h_{k} \in \mathbb{F}_{T}^{2} .
$$

An inspection of the proof of Lemma 6.6(v), and (6.19), (6.22) show that there is a constant $M=M\left(T, h_{0}\right)>0$ such that

$$
\left\|P_{k}\left(h_{1}, \ldots, h_{k}\right)\right\|_{\mathbb{F}_{T}^{2}} \leq M^{k}\left\|h_{1}\right\| \cdots\left\|h_{k}\right\|, \quad h_{1}, \ldots, h_{k} \in \mathbb{F}_{T}^{2} .
$$

This shows that the mapping $P_{k}: \mathbb{F}_{T}^{2} \times \cdots \times \mathbb{F}_{T}^{2} \rightarrow \mathbb{F}_{T}^{2}$ is well-defined, $k$-multilinear, symmetric, and continuous. Moreover, it shows that the series converges in $\mathbb{F}_{T}^{2}$, provided $\left\|h-h_{0}\right\|_{\mathbb{F}_{T}^{2}} \leq r$ with $r$ sufficiently small. We can now conclude that

$$
\left[h \mapsto \sum_{k=1}^{\infty}(-1)^{k}\left(\frac{h-h_{0}}{1+h_{0}}\right)^{k}\right] \in C^{\omega}\left(\mathbb{B}\left(h_{0}, r\right), \mathbb{F}_{T}^{2}\right),
$$

where $\mathbb{B}\left(h_{0}, r\right)$ is the ball of radius $r$ centered at $h_{0}$ in $\mathbb{F}_{T}^{2}$. Using Lemma 6.6(ii),(v) we obtain that

$$
\left[(g, h) \mapsto \frac{g}{1+h}=\frac{g}{1+h_{0}}+\frac{g}{1+h_{0}} \sum_{k=1}^{\infty}(-1)^{k}\left(\frac{h-h_{0}}{1+h_{0}}\right)^{k}\right] \in C^{\omega}\left(\mathbb{F}_{T}^{2} \times \mathbb{B}\left(h_{0}, r\right), \mathbb{F}_{T}^{2}\right)
$$

Since analyticity is a local property, the assertion follows.

Remark 6.7. Let $d$ be a given number. An inspection of the proof of Lemma 6.6(ii) shows that

$$
\|(g+d) h\|_{\mathbb{F}_{T}^{2}} \leq c_{0}\left(\|g+d\|_{\infty}+\|g\|_{\mathbb{F}_{T}^{2}}\right)\|h\|_{\mathbb{F}_{T}^{2}}, \quad(g, h) \in \mathbb{F}_{T}^{2} \times{ }_{0} \mathbb{F}_{T}^{2},
$$

where the constant $c_{0}$ is independent of $T$ for $T \leq T_{0}$.

Acknowledgment: We would like to thank Rico Zacher for the proof of (6.8). The work of this paper was mainly developed during the stay of the second author at the Department of Mathematics at Vanderbilt University. He would like to express his gratitude to the Department for their hospitality. 


\section{REFERENCES}

[1] H. Amann, Linear and Quasilinear Parabolic Problems. Vol. I, Birkhäuser, 1995.

[2] S. Angenent, Nonlinear analytic semiflows. Proc. Roy. Soc. Edinburgh Sect. A 115 (1990), 91-107.

[3] I. Athanasopoulos, L. Caffarelli, S. Salsa, Caloric functions in Lipschitz domains and the regularity of solutions to phase transition problems. Ann. of Math. 143 (1996), 413-434.

[4] I. Athanasopoulos, L. Caffarelli, S. Salsa, Regularity of the free boundary in parabolic phasetransition problems. Acta Math. (2) 176 (1996), 245-282.

[5] I. Athanasopoulos, L. Caffarelli, S. Salsa, Phase transition problems of parabolic type: flat free boundaries are smooth. Comm. Pure Appl. Math 51 (1998), 77-112.

[6] B.V. Bazalǐr, Stability of smooth solutions of a two-phase Stefan problem. (Russian) Dokl. Akad. Nauk SSSR 262 (1982), 265-269; translation in Soviet Math. Dokl. 25 (1982), 46-50.

[7] B.V. Bazaliǔ, The Stefan problem. (Russian) Dokl. Akad. Nauk Ukrain. SSR Ser. A (1986), no. $11,3-7,84$.

[8] B.V. Bazaliǐ, S.P. Degtyarev, On the classical solvability of a multidimensional Stefan problem with convective movement of a viscous incompressible fluid. (Russian) Mat. Sb. (N.S.) 132 (1987); translation in Math. USSR-Sb. 60 (1988), 1-17.

[9] G.I. Bizhanova, Investigation of the solvability in a weighted Hölder function space of the multidimensional two-phase Stefan problem and the multidimensional Florin nonstationary filtration problem for second-order parabolic equations. (Russian) Zap. Nauchn. Sem. S.Peterburg. Otdel. Mat. Inst. Steklov. (POMI) 213 (1994), 14-47; translation in J. Math. Sci. (New York) 84 (1997), 823-844.

[10] G.I. Bizhanova, Solution in a weighted Hölder function space of multidimensional two-phase Stefan and Florin problems for second-order parabolic equations in a bounded domain. (Russian) Algebra i Analiz 7 (1995), 46-76; translation in St. Petersburg Math. J. 7 (1996), $217-241$.

[11] G.I. Bizhanova, V.A. Solonnikov, On the solvability of an initial-boundary value problem for a second-order parabolic equation with a time derivative in the boundary condition in a weighted Hölder space of functions. (Russian) Algebra i Analiz 5 (1993), 109-142; translation in St. Petersburg Math. J. 5 (1994), 97-124.

[12] G.I. Bizhanova, V.A. Solonnikov, Some model problems for second-order parabolic equations with a time derivative in the boundary conditions. (Russian) Algebra i Analiz 6 (1994), 30-50; translation in St. Petersburg Math. J. 6 (1995), 1151-1166.

[13] G.I. Bizhanova, V.A. Solonnikov, On problems with free boundaries for second-order parabolic equations. (Russian) Algebra i Analiz 12 (2000), 98-139; translation in St. Petersburg Math. J. 12 (2001), 949-981.

[14] L.A. Caffarelli, The regularity of free boundaries in higher dimensions. Acta Math. 139 (1977), $155-184$.

[15] L.A. Caffarelli, Some aspects of the one-phase Stefan problem. Indiana Univ. Math. J. 27 (1978), 73-77.

[16] L.A. Caffarelli, L.C. Evans, Continuity of the temperature in the two-phase Stefan problem. Arch. Rational Mech. Anal. 81 (1983), 199-220.

[17] L.A. Caffarelli, A. Friedman, Continuity of the temperature in the Stefan problem. Indiana Univ. Math. J. 28 (1979), 53-70.

[18] E. DiBenedetto, Regularity properties of the solution of an $n$-dimensional two-phase Stefan problem. Boll. Un. Mat. Ital. Suppl. (1980), 129-152.

[19] E. DiBenedetto, Continuity of weak solutions to certain singular parabolic equations. Ann. Mat. Pura Appl. (4) 130 (1982), 131-176.

[20] E. DiBenedetto, A. Friedman, The ill-posed Hele-Shaw model and the Stefan problem for supercooled water. Trans. Amer. Math. Soc. 282 (1984), 183-204.

[21] E. DiBenedetto, V. Vespri, On the singular equation $\beta(u)_{t}=\Delta u$. Arch. Rational Mech. Anal. 132 (1995), 247-309.

[22] G. Duvaut, Résolution d'un problème de Stefan (fusion d'un bloc de glace à zéro degré). C.R. Acad. Sci. Paris Sér. A-B 276 (1973), 1461-1463.

[23] J. Escher, On moving boundaries in deformable media. Adv. Math. Sci. Appl. 7 (1997), $275-316$. 
[24] J. Escher, G. Simonett. Analyticity of the interface in a free boundary problem, Math. Ann. 305 (1996), 439-459.

[25] J. Escher, G. Simonett, Analyticity of solutions to fully nonlinear parabolic evolution equations on symmetric spaces. Dedicated to Philippe Bnilan. J. Evol. Equ. 3 (2003), 549-576.

[26] J. Escher, J. Prss and G. Simonett, A new approach to the regularity of solutions for parabolic equations. Evolution equations. Lecture Notes in Pure and Appl. Math., 234, Dekker, New York, 2003, 167-190.

[27] J. Escher, J. Prüss and G. Simonett, Analytic solutions for a Stefan problem with GibbsThomson correction. J. Reine Angew. Math. (2003), 1-52.

[28] A. Friedman, Partial differential equations of parabolic type. Prentice-Hall, Inc., Englewood Cliffs, N.J. 1964

[29] A. Friedman, The Stefan problem in several space variables. Trans. Amer. Math. Soc. 133 (1968), 51-87.

[30] A. Friedman, Analyticity of the free boundary for the Stefan problem. Arch. Rational Mech. Anal. 61 (1976), 97-125.

[31] A. Friedman, Variational Principles and Free-Boundary Problems, Wiley-Interscience, New York, 1982.

[32] A. Friedman, D. Kinderlehrer, A one phase Stefan problem. Indiana Univ. Math. J. 24 (1975), 1005-1035.

[33] E.V. Frolova, Solvability in Sobolev spaces of a problem for a second order parabolic equation with time derivative in the boundary condition. Portugal. Math. 56 (1999), 419-441.

[34] E.I. Hanzawa, Classical solutions of the Stefan problem, Tôhoku Math. J. 33 (1981), 297-335.

[35] M. Hieber, J. Prüss, Functional calculi for linear operators in vector-valued $L^{p}$-spaces via the transference principle. Adv. Differential Equations 3 (1998), 847-872.

[36] N. Kalton, L. Weis. The $H^{\infty}$-calculus and sums of closed operators. Math. Ann., 321:319-345, 2001.

[37] S.L. Kamenomostskaja, On Stefan's problem. Math. Sbornik 53 (1965), 485-514.

[38] D. Kinderlehrer, L. Nirenberg, The smoothness of the free boundary in the one phase Stefan problem. Comm. Pure Appl. Math. 31 (1978), 257-282.

[39] D. Kinderlehrer, L. Nirenberg, Analyticity at the boundary of solutions of nonlinear secondorder parabolic equations. Comm. Pure Appl. Math. 31 (1978), 283-338.

[40] H. Koch, Classical solutions to phase transition problems are smooth. Comm. Partial Differential Equations 23 (1998), 389-437.

[41] O.A. Ladyženskaya, V.A. Solonnikov, and N.N. Ural'ceva, Linear and Quasilinear Equations of Parabolic Type. Volume 23 of Transl. Math. Monographs. Amer. Math. Soc., 1968.

[42] K. Masuda, On the regularity of solutions of the nonstationary Navier-Stokes equations. Approximation methods for Navier-Stokes problems. (Proc. Sympos., Univ. Paderborn, Paderborn, 1979). Lecture Notes in Math., 771, Springer, Berlin, 1980, 360-370.

[43] H. Matano, Asymptotic behavior of the free boundaries arising in one phase Stefan problems in multi-dimensional spaces. Lecture Notes in Num. Appl. Anal. 5, Kinokuniya, Tokyo, 1982, $133-151$.

[44] A.M. Meirmanov, On the classical solution of the multidimensional Stefan problem for quasilinear parabolic equations. Math. Sb. 112 (1980), 170-192.

[45] A.M. Meirmanov, The Stefan Problem, De Gruyter, Berlin, 1992.

[46] R.H. Nochetto, A class of nondegenerate two-phase Stefan problems in several space variables. Comm. Partial Differential Equations 12 (1987), 21-45.

[47] J. Prüss, Evolutionary Integral Equations and Applications. Volume 87 of Monographs in Mathematics. Birkhäuser Verlag, Basel, 1993.

[48] J. Prüss and H. Sohr, On operators with bounded imaginary powers in Banach spaces. Math. Z. 203 (1990), 429-452.

[49] E.V. Radkevich, On the solvability of general nonstationary problems with a free boundary. (Russian) Dokl. Akad. Nauk SSSR 288 (1986), 1094-1099.

[50] L.I. Rubinstein, The Stefan Problem. Translations of Mathematical Monographs, Vol. 27, American Mathematical Society, Providence, R.I. 1971.

[51] P.E. Sacks, Continuity of solutions of a singular parabolic equation. Nonlinear Anal. 7 (1983), 387-409. 
[52] V.A. Solonnikov, Free boundary problems for second order parabolic equations. Progress in partial differential equations, Vol. 2 (Pont--Mousson, 1997). Pitman Res. Notes Math. Ser., 384, Longman, Harlow, 1998, 116-126.

[53] V.A. Solonnikov, E.V. Frolova, $L_{p}$-theory for the Stefan problem. Journal of Mathematical Sciences 99, No. 1 (2000), 989-1006.

[54] H. Triebel, Interpolation Theory, Function Spaces, Differential Operators North-Holland, Amsterdam, 1978

[55] H. Triebel, Theory of Function Spaces, Volume 78 of Monographs in Mathematics. Birkhäuser, Basel, 1983.

[56] A. Visintin, Models of Phase Transitions. Progress in Nonlinear Differential Equations and Their Applications, Volume 28, Birkhäuser, Boston, 1996.

[57] W.P. Ziemer, Interior and boundary continuity of weak solutions of degenerate parabolic equations. Trans. Amer. Math. Soc. 271 (1982), 733-748.

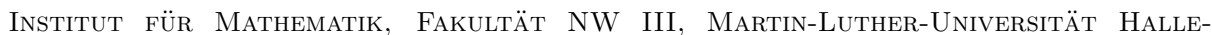
Wittenberg, D-60120 Halle, Germany

E-mail address: jan.pruess@mathematik.uni-halle.de

Department of Mathematics and Statistics, University of Konstanz, D-78457 KonSTANZ, Germany

E-mail address: Juergen.Saal@uni-konstanz.de

Department of Mathematics, Vanderbilt University, Nashville, TN 37240, USA

E-mail address: gieri.simonett@vanderbilt.edu 